\title{
Catalyst-Controlled Divergent Intramolecular Cyclizations of Morita-Baylis-Hillman Carbonates
}

\author{
Wei Cai, Yanlin He, Yiming Zhou, and You Huang* \\ State Key Laboratory and Institute of Elemento-Organic Chemistry, College of Chemistry, Nankai University, \\ Tianjin 300071, People's Republic of China
}

\section{CONTENTS}

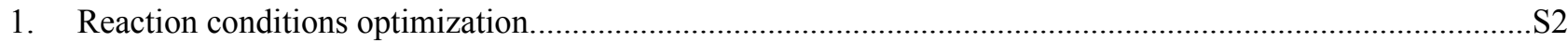

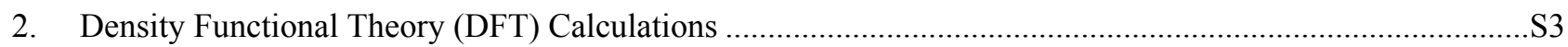

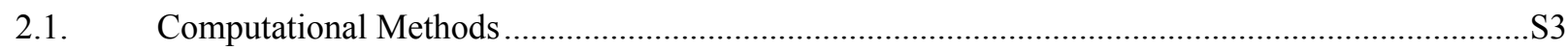

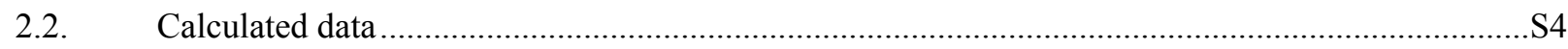

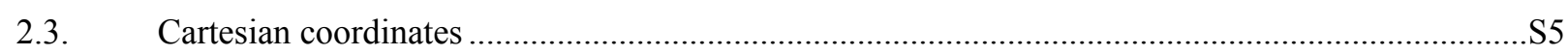

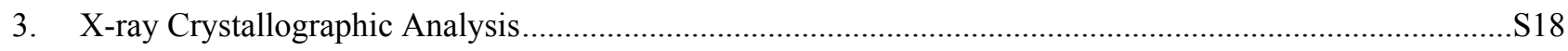

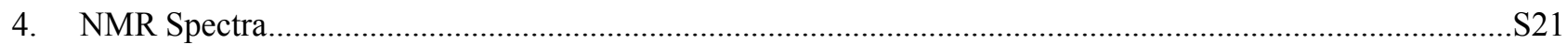

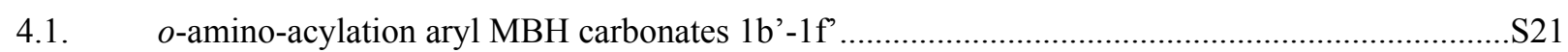

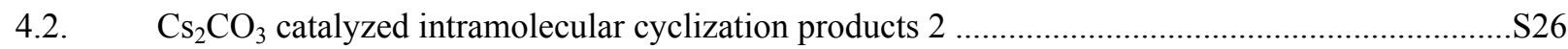

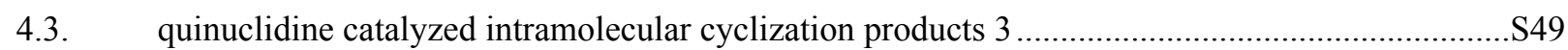

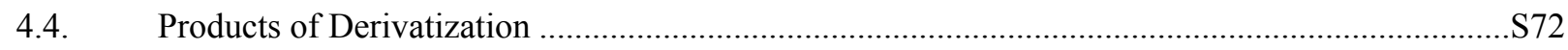




\section{Reaction conditions optimization.}

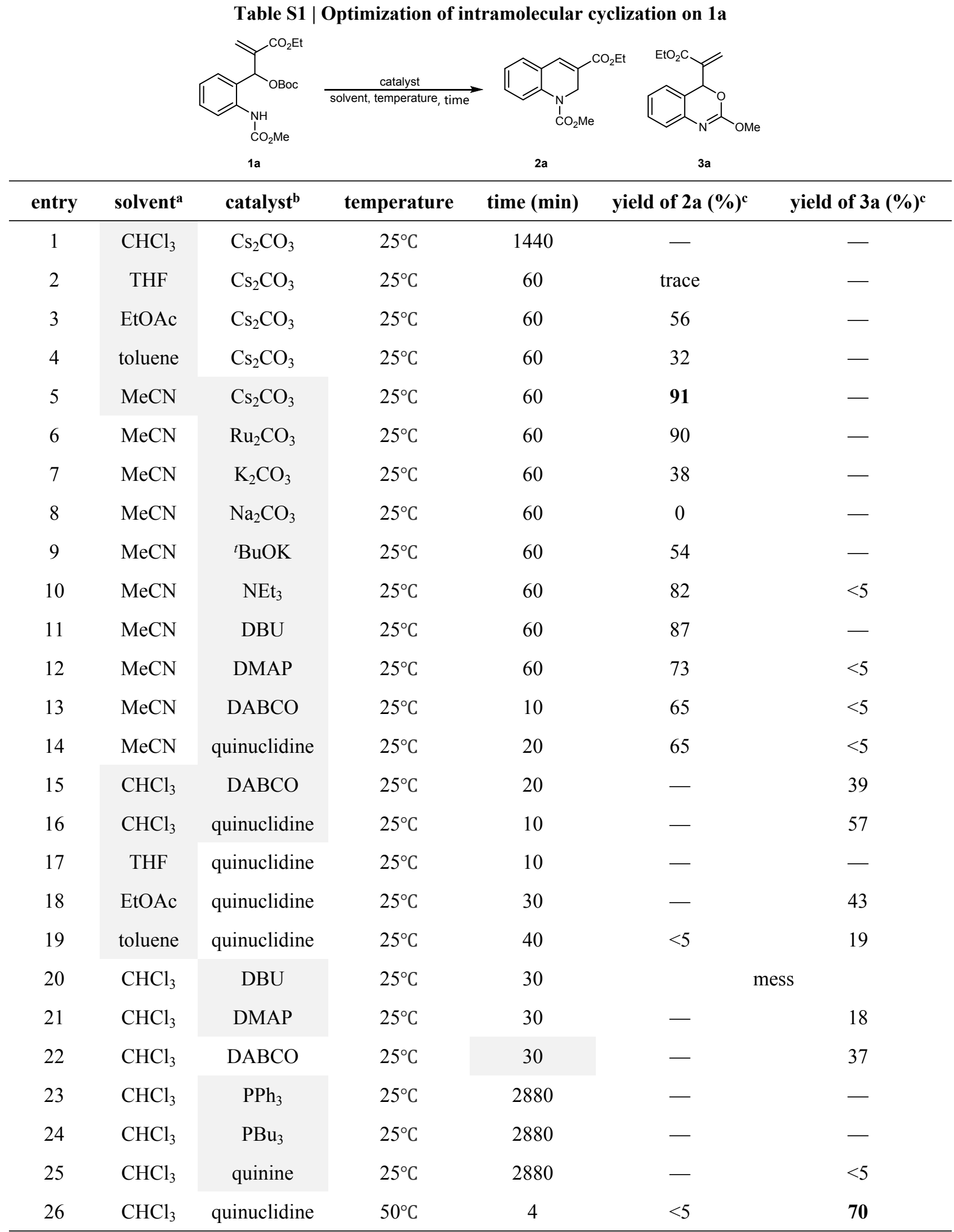

${ }^{\mathrm{a}} 1 \mathrm{~mL},{ }^{\mathrm{b}} 0.01 \mathrm{mmol},{ }^{\mathrm{c}}$ Isolated yield. 


\section{Density Functional Theory (DFT) Calculations}

\subsection{Computational Methods}

Density functional theory (DFT) investigations were performed to delineate the detailed mechanism of the Bronsted base/Lewis base-catalyzed intramolecular cyclizations of $o$-amino-acylation aryl MBH carbonates. All DFT calculations were carried out with the Gaussian 09 programs $^{1}$. The geometry optimizations of the reactants, transition states, and products were performed with the M06-2X/6-31G(d) level ${ }^{2}$. The frequency calculations were carried out at the same level to conform that there was no imaginary frequency for ground state structures and only one imaginary frequency for transition state structures. The solvent $\left(\mathrm{MeCN}\right.$ or $\left.\mathrm{CHCl}_{3}\right)$ effects were considered by single point energy calculations on the gas-phase stationary points using M06-2X functional with the def2-TZVP ${ }^{3}$ basis set in a SMD continuum solvation model. The energies given in this work are M06-2X calculated Gibbs free energies in $\mathrm{MeCN}$ or $\mathrm{CHCl}_{3}$ solvent noted on figures $^{4}$. In addition, we utilized a correction of $(\mathrm{n}-\mathrm{m})^{*} 1.89 \mathrm{kcal} / \mathrm{mol}$ for a process from $\mathrm{m}$ - to $\mathrm{n}$-components to account for the Gibbs energy transition from gas to solution.

\footnotetext{
${ }^{1}$ Gaussian 09, Revision D.01, Frisch, M. J.; Trucks, G. W.; Schlegel, H. B.; Scuseria, G. E.; Robb, M. A.; Cheeseman, J. R.; Scalmani, G.; Barone, V.; Mennucci, B.; Petersson, G. A.; Nakatsuji, H.; Caricato, M.; Li, X.; Hratchian, H. P.; Izmaylov, A. F.; Bloino, J.; Zheng, G.; Sonnenberg, J. L.; Hada, M.; Ehara, M.; Toyota, K.; Fukuda, R.; Hasegawa, J.; Ishida, M.; Nakajima, T.; Honda, Y.; Kitao, O.; Nakai, H.; Vreven, T.; Montgomery, Jr., J. A.; Peralta, J. E.; Ogliaro, F.; Bearpark, M.; Heyd, J. J.; Brothers, E.; Kudin, K. N.; Staroverov, V. N.; Kobayashi, R.; Normand, J.; Raghavachari, K.; Rendell, A.; Burant, J. C.; Iyengar, S. S.; Tomasi, J.; Cossi, M.; Rega, N.; Millam, N. J.; Klene, M.; Knox, J. E.; Cross, J. B.; Bakken, V.; Adamo, C.; Jaramillo, J.; Gomperts, R.; Stratmann, R. E.; Yazyev, O.; Austin, A. J.; Cammi, R.; Pomelli, C.; Ochterski, J. W.; Martin, R. L.; Morokuma, K.; Zakrzewski, V. G.; Voth, G. A.; Salvador, P.; Dannenberg, J. J.; Dapprich, S.; Daniels, A. D.; Farkas, Ö.; Foresman, J. B.; Ortiz, J. V.; Cioslowski, J.; Fox, D. J. Gaussian, Inc., Wallingford CT, 2009.

2 (a) Zhao, Y. \& Truhlar, D. G. Density functionals with broad applicability in chemistry. Acc. Chem. Res. 2008, 41, 157-167; (b) Zhao, Y. \& Truhlar, D. G. The M06 suite of density functionals for main group thermochemistry, thermochemical kinetics, noncovalent interactions, excited states, and transition elements: Two new functionals and systematic testing of four M06-class functionals and 12 other functionals. Theor. Chem. Acc. 2008, 120, 215-241; (c) Becke, A. D. Density-functional thermochemistry. III. The role of exact exchange. J. Chem. Phys. 1993, 98, 5648-5652; (d) Lee, C.; Yang, W.; Parr, R. G. Development of the Colle-Salvetti correlationenergy formula into a functional of the electron density. Phys. Rev. B 1988, 37, 785-789.

${ }^{3}$ Weigend, F. \& Ahlrichs, R. Balanced basis sets of split valence, triple zeta valence and quadruple zeta valence quality for H to Rn: Design and assessment of accuracy. Phys. Chem. Chem. Phys. 2005, 7, 3297-305.

${ }^{4}$ Marenich, A. V.; Cramer, C. J.; Truhlar, D. G., Universal Solvation Model Based on Solute Electron Density and on a Continuum Model of the Solvent Defined by the Bulk Dielectric Constant and Atomic Surface Tensions. J. Phys. Chem. B. 2009, 113, 6378-6396.
} 


\subsection{Calculated data}

Table S2 | The absolute calculation energies, enthalpies and free energies of $\mathrm{Cs}_{2} \mathrm{CO}_{3}$ catalyzed intramolecular cyclization.

\begin{tabular}{|l|l|l|l|l|l|}
\hline Geometry & $\mathrm{E}_{(\mathrm{elec})}{ }^{1}$ & $\mathrm{E}_{(\mathrm{solv})}{ }^{2}$ & $\mathrm{G}_{(\mathrm{corr})}{ }^{3}$ & $\mathrm{H}_{(\mathrm{corr})}{ }^{4}$ & $\mathrm{IF}^{5}$ \\
\hline $\mathbf{1 b}$ & -1280.262547 & -1281.14422411 & 0.344924 & 0.431572 & \\
\hline $\mathbf{C O}_{\mathbf{3}}{ }^{-}$ & -263.508618 & -264.033491015 & -0.011079 & 0.018582 & \\
\hline $\mathbf{H C O}_{3}{ }^{-}$ & -264.327898 & -264.567531234 & 0.001345 & 0.031491 & \\
\hline $\mathbf{C O}_{2}$ & -188.517761 & -188.596837844 & -0.008735 & 0.015525 & \\
\hline tBuO- & -232.826769 & -233.129924515 & 0.093301 & 0.129250 & \\
\hline INT1 & -1279.705195 & -1280.64216088 & 0.330169 & 0.416820 & \\
\hline TS2 & -1279.691217 & -1280.62114194 & 0.332680 & 0.415948 & -259.69 \\
\hline INT2 & -1279.716388 & -1280.65238617 & 0.333953 & 0.418362 & \\
\hline TS3 & -1279.714621 & -1280.65131547 & 0.335562 & 0.417387 & -14.32 \\
\hline $\mathbf{2}$ & -858.328993 & -858.890223335 & 0.204516 & 0.266137 & \\
\hline
\end{tabular}

${ }^{a}$ The electronic energy calculated by M06-2x/6-31G(d) level in gas phase. ${ }^{b}$ The solvation energy corrections calculated at the M06-2x/def2-TZVP level with the SMD solvation model for acetonitrile minus electronic energy in gas phase. ${ }^{\mathrm{c}}$ The thermal correction to Gibbs free energy calculated by M06-2x/6-31G(d) level in gas phase. ${ }^{\mathrm{d}}$ The thermal correction to enthalpy calculated by M06-2x/6-31G(d) level in gas phase. ${ }^{\mathrm{e}}$ The M06-2x/6-31G(d) level calculated imaginary frequencies for the transition states.

Table S3 | The absolute calculation energies, enthalpies and free energies of DABCO catalyzed intramolecular cyclization.

\begin{tabular}{|l|l|l|l|l|l|}
\hline Geometry & $\mathrm{E}_{(\mathrm{elec})}{ }^{1}$ & $\left.\mathrm{E}_{(\mathrm{solv})}\right)^{2}$ & $\mathrm{G}_{(\mathrm{corr})}{ }^{3}$ & $\mathrm{H}_{(\mathrm{corr})}{ }^{4}$ & $\mathrm{IF}^{5}$ \\
\hline 1b & -1280.262547 & -1281.1411271 & 0.344924 & 0.431572 & \\
\hline CO' & -188.517761 & -188.594609085 & -0.008735 & 0.015525 & \\
\hline tBuO- & -232.826769 & -233.115475297 & 0.093301 & 0.129250 & \\
\hline tBuOH & -233.442156 & -233.660746667 & 0.108428 & 0.145078 & \\
\hline DABCO & -345.018414 & -345.313541488 & 0.155886 & 0.193762 & \\
\hline INT3 & -1203.291034 & -1204.1723119 & 0.384822 & 0.463060 & \\
\hline TS4 & -1203.266085 & -1204.15942151 & 0.385258 & 0.461003 & -224.92 \\
\hline INT4 & -1203.276715 & -1204.16096906 & 0.385862 & 0.462438 & \\
\hline TS5 & -1203.276702 & -1204.15316365 & 0.383895 & 0.460565 & -180.79 \\
\hline 3 & -858.286602 & -858.84736183 & 0.202030 & 0.264838 & \\
\hline TS4' & -1203.257615 & -1204.14058133 & 0.384700 & 0.460458 & -246.16 \\
\hline INT4' & -1203.262926 & -1204.14299017 & 0.383611 & 0.461606 & \\
\hline TS5 & -1203.263019 & -1204.14008259 & 0.383932 & 0.460203 & -131.39 \\
\hline 3' & -858.273265 & -858.829628132 & 0.200694 & 0.264350 & \\
\hline
\end{tabular}

${ }^{a}$ The electronic energy calculated by M06-2x/6-31G(d) level in gas phase. ${ }^{b}$ The solvation energy corrections calculated at the M06-2x/def2-TZVP level with the SMD solvation model for acetonitrile minus electronic energy in gas phase. ${ }^{\mathrm{c}}$ The thermal correction to Gibbs free energy calculated by M06-2x/6-31G(d) level in gas phase. ${ }^{\mathrm{d}}$ The thermal correction to enthalpy calculated by M06-2x/6-31G(d) level in gas phase. ${ }^{\mathrm{e}}$ The M06-2x/6-31G(d) level calculated imaginary frequencies for the transition states. 
2.3. Cartesian coordinates

\begin{tabular}{|c|c|c|c|}
\hline \multicolumn{4}{|c|}{$1 b$} \\
\hline $\mathrm{C}$ & 0.52354600 & -0.35756700 & 1.05500700 \\
\hline $\mathrm{C}$ & 1.14813100 & 0.89664800 & 1.10628300 \\
\hline $\mathrm{C}$ & 1.30897200 & 1.53513200 & 2.34205400 \\
\hline $\mathrm{C}$ & 0.86806000 & 0.92318400 & 3.50656500 \\
\hline $\mathrm{C}$ & 0.27868000 & -0.33911100 & 3.46622600 \\
\hline $\mathrm{C}$ & 0.11482500 & -0.96948300 & 2.24086900 \\
\hline $\mathrm{H}$ & 1.77049700 & 2.51381000 & 2.36961300 \\
\hline $\mathrm{H}$ & 0.99946000 & 1.43243300 & 4.45645600 \\
\hline $\mathrm{H}$ & -0.05340600 & -0.82286700 & 4.37913200 \\
\hline $\mathrm{H}$ & -0.36054100 & -1.94567700 & 2.18229400 \\
\hline $\mathrm{N}$ & 1.63489100 & 1.47560400 & -0.07905900 \\
\hline $\mathrm{H}$ & 2.06637700 & 0.85610900 & -0.75689100 \\
\hline $\mathrm{C}$ & 1.70052300 & 2.81661700 & -0.32778000 \\
\hline $\mathrm{O}$ & 1.32684700 & 3.71272600 & 0.39484300 \\
\hline $\mathrm{O}$ & 2.26366300 & 3.01270900 & -1.54164900 \\
\hline $\mathrm{C}$ & 0.20286800 & -1.06156100 & -0.25449200 \\
\hline $\mathrm{H}$ & 0.37598700 & -0.38267200 & -1.09349200 \\
\hline $\mathrm{O}$ & -1.17402100 & -1.45282700 & -0.26199900 \\
\hline $\mathrm{C}$ & 1.00956800 & -2.32572000 & -0.43123900 \\
\hline $\mathrm{C}$ & 0.51238800 & -3.55929300 & -0.35684700 \\
\hline $\mathrm{H}$ & -0.54597600 & -3.71918800 & -0.18478900 \\
\hline $\mathrm{H}$ & 1.15692100 & -4.42267000 & -0.47753500 \\
\hline $\mathrm{C}$ & 2.37575000 & 4.38087000 & -1.91579000 \\
\hline $\mathrm{H}$ & 2.86100200 & 4.37984000 & -2.89121300 \\
\hline $\mathrm{H}$ & 1.38829500 & 4.84367400 & -1.97995500 \\
\hline $\mathrm{H}$ & 2.97709600 & 4.93124700 & -1.18860000 \\
\hline $\mathrm{C}$ & 2.44853000 & -2.07485700 & -0.71619200 \\
\hline $\mathrm{O}$ & 2.89811400 & -0.97119500 & -0.95147100 \\
\hline $\mathrm{O}$ & 3.20178700 & -3.17555800 & -0.70852700 \\
\hline $\mathrm{C}$ & 4.58401000 & -2.96483800 & -1.00388200 \\
\hline $\mathrm{H}$ & 5.02351100 & -2.27777700 & -0.27821100 \\
\hline $\mathrm{H}$ & 5.05027000 & -3.94622700 & -0.93941400 \\
\hline $\mathrm{H}$ & 4.69760100 & -2.54576800 & -2.00579300 \\
\hline $\mathrm{C}$ & -2.03962800 & -0.44295600 & -0.46879600 \\
\hline $\mathrm{O}$ & -1.72066100 & 0.68764600 & -0.73752600 \\
\hline $\mathrm{O}$ & -3.26320500 & -0.93795600 & -0.33131000 \\
\hline $\mathrm{C}$ & -4.41752800 & -0.05793600 & -0.49148600 \\
\hline $\mathrm{C}$ & -4.38396000 & 1.04030100 & 0.56633100 \\
\hline $\mathrm{C}$ & -4.45772200 & 0.50316100 & -1.90925100 \\
\hline $\mathrm{C}$ & -5.58841500 & -1.00321600 & -0.25256000 \\
\hline $\mathrm{H}$ & -4.28660800 & 0.59726600 & 1.56222600 \\
\hline $\mathrm{H}$ & -3.55414300 & 1.72824500 & 0.39907300 \\
\hline $\mathrm{H}$ & -5.32194700 & 1.60292800 & 0.53062800 \\
\hline $\mathrm{H}$ & -4.41216100 & -0.31284600 & -2.63736100 \\
\hline $\mathrm{H}$ & -5.39988300 & 1.04034600 & -2.05564400 \\
\hline $\mathrm{H}$ & -3.62966900 & 1.19042200 & -2.08651000 \\
\hline $\mathrm{H}$ & -6.53191900 & -0.45714100 & -0.33935900 \\
\hline $\mathrm{H}$ & -5.58131100 & -1.81273700 & -0.98801100 \\
\hline $\mathrm{H}$ & -5.52547800 & -1.44059600 & 0.74774600 \\
\hline \multicolumn{4}{|c|}{$\mathrm{CO}_{3}{ }^{2-}$} \\
\hline $\mathrm{C}$ & -0.00006100 & -0.00002800 & 0.00005200 \\
\hline $\mathrm{O}$ & 0.42081400 & 1.23499900 & 0.00000500 \\
\hline $\mathrm{O}$ & 0.85923700 & -0.98188200 & 0.00000200 \\
\hline $\mathrm{O}$ & -1.28000500 & -0.25309600 & -0.00004600 \\
\hline \multicolumn{4}{|c|}{$\mathrm{HCO}_{3}^{-}$} \\
\hline
\end{tabular}




\begin{tabular}{|c|c|c|c|}
\hline $\mathrm{O}$ & 0.17692700 & 1.27961700 & -0.00004500 \\
\hline $\mathrm{O}$ & 0.97228200 & -0.81740200 & -0.00001000 \\
\hline $\mathrm{O}$ & -1.24841900 & -0.49423200 & -0.00011800 \\
\hline $\mathrm{H}$ & 1.70697600 & -0.18841500 & 0.00000000 \\
\hline \multicolumn{4}{|c|}{$\mathrm{CO}_{2}$} \\
\hline $\mathrm{C}$ & 0.00000000 & 0.00000000 & 0.00000000 \\
\hline $\mathrm{O}$ & 0.00000000 & 0.00000000 & 1.16279700 \\
\hline $\mathrm{O}$ & 0.00000000 & 0.00000000 & -1.16279700 \\
\hline \multicolumn{4}{|c|}{${ }^{t} \mathrm{BuO}^{-}$} \\
\hline $\mathrm{C}$ & -0.89339343 & 1.96696694 & 0.00000000 \\
\hline $\mathrm{C}$ & -1.27465743 & 3.36700494 & 0.59367300 \\
\hline $\mathrm{H}$ & 0.09895457 & 1.69114894 & 0.37936700 \\
\hline $\mathrm{H}$ & -1.60874843 & 1.22585494 & 0.37924800 \\
\hline $\mathrm{H}$ & -0.87855943 & 1.91239694 & -1.10161700 \\
\hline $\mathrm{C}$ & -2.67803743 & 3.73653494 & 0.00015500 \\
\hline $\mathrm{C}$ & -0.25303443 & 4.39702994 & -0.00079700 \\
\hline $\mathrm{H}$ & -2.96183143 & 4.72693494 & 0.37882700 \\
\hline $\mathrm{H}$ & -2.73314343 & 3.75020494 & -1.10150100 \\
\hline $\mathrm{H}$ & -3.41309843 & 3.01534694 & 0.38016300 \\
\hline $\mathrm{H}$ & -0.21237243 & 4.43641194 & -1.10245500 \\
\hline $\mathrm{H}$ & -0.51056143 & 5.39469694 & 0.37743000 \\
\hline $\mathrm{H}$ & 0.74616557 & 4.14848494 & 0.37936400 \\
\hline $\mathrm{O}$ & -1.27398443 & 3.36749594 & 1.91614800 \\
\hline \multicolumn{4}{|c|}{ INT1 } \\
\hline $\mathrm{C}$ & -0.77272700 & -0.25967500 & 1.19063200 \\
\hline $\mathrm{C}$ & -0.99803300 & -0.92673900 & 2.39152900 \\
\hline $\mathrm{C}$ & -1.27973200 & -0.23498000 & 3.56460100 \\
\hline $\mathrm{C}$ & -1.32419700 & 1.15881100 & 3.51027600 \\
\hline $\mathrm{C}$ & -1.09536500 & 1.84879400 & 2.32931400 \\
\hline $\mathrm{C}$ & -0.80828000 & 1.16565100 & 1.11566600 \\
\hline $\mathrm{H}$ & -1.45289700 & -0.76688600 & 4.49572900 \\
\hline $\mathrm{H}$ & -1.54084800 & 1.72544400 & 4.41491300 \\
\hline $\mathrm{H}$ & -1.13075400 & 2.92933400 & 2.30421500 \\
\hline $\mathrm{N}$ & -0.57280900 & 1.72115000 & -0.10968400 \\
\hline $\mathrm{C}$ & -0.58556600 & 3.03924700 & -0.28545800 \\
\hline $\mathrm{O}$ & -0.71296100 & 3.99922800 & 0.48144400 \\
\hline $\mathrm{O}$ & -0.40491900 & 3.31575900 & -1.64471700 \\
\hline $\mathrm{C}$ & -0.54039600 & -1.10328300 & -0.04357500 \\
\hline $\mathrm{H}$ & -0.46341600 & -2.14719800 & 0.27383200 \\
\hline $\mathrm{O}$ & 0.68411600 & -0.77352600 & -0.73923700 \\
\hline $\mathrm{C}$ & -1.66443400 & -0.99656200 & -1.04851900 \\
\hline $\mathrm{C}$ & -1.69073400 & -0.12835800 & -2.06161500 \\
\hline $\mathrm{H}$ & -0.87658600 & 0.57497700 & -2.19115700 \\
\hline $\mathrm{H}$ & -2.54489500 & -0.09102300 & -2.73021500 \\
\hline $\mathrm{C}$ & -0.38572900 & 4.68615300 & -1.95093000 \\
\hline $\mathrm{H}$ & -0.24366800 & 4.75666700 & -3.03373700 \\
\hline $\mathrm{H}$ & -1.32157800 & 5.18122400 & -1.66678700 \\
\hline $\mathrm{H}$ & 0.42963100 & 5.20733000 & -1.43629200 \\
\hline $\mathrm{C}$ & -2.78533800 & -1.93311100 & -0.79362100 \\
\hline $\mathrm{O}$ & -2.78338700 & -2.81639200 & 0.03610100 \\
\hline $\mathrm{O}$ & -3.85499900 & -1.72276700 & -1.59940500 \\
\hline $\mathrm{C}$ & -4.94483400 & -2.59855300 & -1.36509500 \\
\hline $\mathrm{H}$ & -5.29428200 & -2.51350200 & -0.33285400 \\
\hline $\mathrm{H}$ & -5.72816100 & -2.29296500 & -2.05952100 \\
\hline $\mathrm{H}$ & -4.65712800 & -3.63789500 & -1.54804900 \\
\hline $\mathrm{C}$ & 1.79539300 & -1.16533000 & -0.14263600 \\
\hline $\mathrm{O}$ & 1.87817300 & -1.85842600 & 0.84627100 \\
\hline $\mathrm{O}$ & 2.82959700 & -0.68086000 & -0.85209500 \\
\hline $\mathrm{C}$ & 4.17641500 & -0.87821900 & -0.36889700 \\
\hline $\mathrm{C}$ & 4.34911600 & -0.23677500 & 1.00644700 \\
\hline $\mathrm{C}$ & 4.53259700 & -2.36387900 & -0.35675300 \\
\hline
\end{tabular}




\begin{tabular}{|c|c|c|c|}
\hline $\mathrm{C}$ & 5.01583200 & -0.13934800 & -1.40674100 \\
\hline $\mathrm{H}$ & 4.02108100 & 0.80648200 & 0.97116000 \\
\hline $\mathrm{H}$ & 3.76394700 & -0.76398600 & 1.76049400 \\
\hline $\mathrm{H}$ & 5.40700800 & -0.26123900 & 1.29014300 \\
\hline $\mathrm{H}$ & 4.34755300 & -2.79878600 & -1.34446900 \\
\hline $\mathrm{H}$ & 5.59589900 & -2.48273900 & -0.12121800 \\
\hline $\mathrm{H}$ & 3.93861100 & -2.89790600 & 0.38542300 \\
\hline H & 6.07782400 & -0.20483500 & -1.14957800 \\
\hline $\mathrm{H}$ & 4.86354700 & -0.57709400 & -2.39776300 \\
\hline $\mathrm{H}$ & 4.72252100 & 0.91332800 & -1.44522600 \\
\hline $\mathrm{H}$ & -0.95263100 & -2.01399400 & 2.39410400 \\
\hline \multicolumn{4}{|c|}{ TS2 } \\
\hline C & -0.15618000 & 0.48527700 & 0.68037500 \\
\hline $\mathrm{C}$ & -0.91238000 & 1.12618000 & 1.66167700 \\
\hline $\mathrm{C}$ & -0.97381800 & 0.66119500 & 2.96917800 \\
\hline C & -0.22984700 & -0.47214700 & 3.30305500 \\
\hline $\mathrm{C}$ & 0.57422900 & -1.10148300 & 2.36577700 \\
\hline $\mathrm{C}$ & 0.64214700 & -0.64653500 & 1.02462200 \\
\hline $\mathrm{H}$ & -1.58435700 & 1.16989900 & 3.71036200 \\
\hline $\mathrm{H}$ & -0.26605200 & -0.86610900 & 4.31673400 \\
\hline $\mathrm{H}$ & 1.15652700 & -1.97240700 & 2.63499400 \\
\hline $\mathrm{N}$ & 1.45024500 & -1.18917900 & 0.05577000 \\
\hline $\mathrm{C}$ & 2.10612500 & -2.34261500 & 0.20341000 \\
\hline $\mathrm{O}$ & 2.04687400 & -3.21513800 & 1.06789200 \\
\hline $\mathrm{O}$ & 2.96369900 & -2.52061300 & -0.87157800 \\
\hline $\mathrm{C}$ & -0.11274300 & 1.11363200 & -0.69713400 \\
\hline $\mathrm{H}$ & -0.66802600 & 2.05479200 & -0.68382400 \\
\hline $\mathrm{O}$ & -0.68007400 & 0.25361100 & -1.72783800 \\
\hline $\mathrm{C}$ & 1.33363600 & 1.31761900 & -1.05446400 \\
\hline $\mathrm{C}$ & 2.01981400 & 0.20090600 & -1.45159000 \\
\hline $\mathrm{H}$ & 1.55461400 & -0.52670100 & -2.10068400 \\
\hline $\mathrm{H}$ & 3.10522700 & 0.19215400 & -1.42506500 \\
\hline $\mathrm{C}$ & 3.66885100 & -3.73945100 & -0.84585900 \\
\hline $\mathrm{H}$ & 4.29848800 & -3.74341700 & -1.73901300 \\
\hline $\mathrm{H}$ & 4.29088200 & -3.82487500 & 0.05113000 \\
\hline $\mathrm{H}$ & 2.98879700 & -4.59728500 & -0.86449200 \\
\hline $\mathrm{C}$ & 1.95312200 & 2.47284300 & -0.45837200 \\
\hline $\mathrm{O}$ & 1.37468300 & 3.40486300 & 0.08118900 \\
\hline $\mathrm{O}$ & 3.31969100 & 2.48828400 & -0.59048500 \\
\hline $\mathrm{C}$ & 3.94213600 & 3.61072500 & -0.00159600 \\
\hline $\mathrm{H}$ & 3.72554800 & 3.66706800 & 1.06929300 \\
\hline $\mathrm{H}$ & 5.01413000 & 3.48079200 & -0.16260500 \\
\hline $\mathrm{H}$ & 3.60122000 & 4.54152400 & -0.46548100 \\
\hline $\mathrm{C}$ & -1.91880200 & -0.20269900 & -1.62583100 \\
\hline $\mathrm{O}$ & -2.31908000 & -1.12785600 & -2.29100400 \\
\hline $\mathrm{O}$ & -2.66116800 & 0.50795900 & -0.75552900 \\
\hline $\mathrm{C}$ & -3.88699400 & -0.06699900 & -0.23594400 \\
\hline $\mathrm{C}$ & -4.93239100 & -0.19994200 & -1.34214200 \\
\hline $\mathrm{C}$ & -3.57318100 & -1.39950000 & 0.44026300 \\
\hline $\mathrm{C}$ & -4.34336700 & 0.95511300 & 0.80034300 \\
\hline $\mathrm{H}$ & -5.07670200 & 0.76785100 & -1.83404300 \\
\hline $\mathrm{H}$ & -4.62159000 & -0.93306400 & -2.08564200 \\
\hline $\mathrm{H}$ & -5.88725400 & -0.50840100 & -0.90247900 \\
\hline $\mathrm{H}$ & -2.77139100 & -1.25740200 & 1.17351000 \\
\hline $\mathrm{H}$ & -4.46591600 & -1.76591800 & 0.95853400 \\
\hline $\mathrm{H}$ & -3.25873200 & -2.14490700 & -0.29232200 \\
\hline $\mathrm{H}$ & -5.30910500 & 0.65306500 & 1.21819600 \\
\hline $\mathrm{H}$ & -3.61158400 & 1.02290000 & 1.60918500 \\
\hline $\mathrm{H}$ & -4.45288100 & 1.94080900 & 0.33717900 \\
\hline $\mathrm{H}$ & -1.47271700 & 2.01229700 & 1.37036200 \\
\hline
\end{tabular}




\begin{tabular}{|c|c|c|c|}
\hline $\mathrm{C}$ & -0.34778600 & -0.67000800 & -1.31353900 \\
\hline $\mathrm{C}$ & 0.31176900 & -1.25147500 & -2.39608800 \\
\hline $\mathrm{C}$ & -0.15604100 & -2.41797300 & -2.99312500 \\
\hline $\mathrm{C}$ & -1.31933500 & -2.99983400 & -2.50536600 \\
\hline $\mathrm{C}$ & -1.99966900 & -2.43709600 & -1.43002300 \\
\hline $\mathrm{C}$ & -1.51298500 & -1.28188700 & -0.80461600 \\
\hline $\mathrm{H}$ & 0.37447900 & -2.85685100 & -3.83363100 \\
\hline $\mathrm{H}$ & -1.71772400 & -3.90135300 & -2.96402000 \\
\hline $\mathrm{H}$ & -2.90390600 & -2.90045100 & -1.06577800 \\
\hline $\mathrm{N}$ & -2.18909600 & -0.69137900 & 0.29462700 \\
\hline $\mathrm{C}$ & -2.98109000 & -1.43868700 & 1.11417900 \\
\hline $\mathrm{O}$ & -3.23495400 & -2.62916100 & 1.04582000 \\
\hline $\mathrm{O}$ & -3.50985000 & -0.66979200 & 2.10750400 \\
\hline C & 0.10193600 & 0.66464800 & -0.77816600 \\
\hline $\mathrm{H}$ & 0.66156200 & 1.22548200 & -1.52710100 \\
\hline $\mathrm{O}$ & 1.10262300 & 0.37755900 & 0.35089300 \\
\hline $\mathrm{C}$ & -1.02029400 & 1.42449400 & -0.26705600 \\
\hline $\mathrm{C}$ & -1.89829000 & 0.73006000 & 0.70520300 \\
\hline $\mathrm{H}$ & -1.45724100 & 0.65100500 & 1.71105500 \\
\hline $\mathrm{H}$ & -2.85113900 & 1.25001400 & 0.80875000 \\
\hline $\mathrm{C}$ & -4.33875800 & -1.38507300 & 3.00384100 \\
\hline $\mathrm{H}$ & -4.70423100 & -0.64741700 & 3.71931500 \\
\hline $\mathrm{H}$ & -5.17441200 & -1.85272200 & 2.47638200 \\
\hline $\mathrm{H}$ & -3.77589200 & -2.16846200 & 3.51863500 \\
\hline $\mathrm{C}$ & -1.15833200 & 2.76995500 & -0.62041000 \\
\hline $\mathrm{O}$ & -0.44437000 & 3.45850800 & -1.35831600 \\
\hline $\mathrm{O}$ & -2.29124300 & 3.36740600 & -0.04170100 \\
\hline $\mathrm{C}$ & -2.49053700 & 4.70129600 & -0.43644700 \\
\hline $\mathrm{H}$ & -2.64841600 & 4.78803400 & -1.51771100 \\
\hline $\mathrm{H}$ & -3.38177600 & 5.04828000 & 0.09553300 \\
\hline $\mathrm{H}$ & -1.63303900 & 5.33159500 & -0.17806400 \\
\hline $\mathrm{C}$ & 2.34573600 & 0.18025200 & 0.00426700 \\
\hline $\mathrm{O}$ & 2.81351000 & 0.18337800 & -1.11851600 \\
\hline $\mathrm{O}$ & 3.07253400 & -0.02910200 & 1.13032500 \\
\hline $\mathrm{C}$ & 4.49357500 & -0.24674800 & 1.03279500 \\
\hline $\mathrm{C}$ & 4.79852600 & -1.50890000 & 0.22671700 \\
\hline $\mathrm{C}$ & 5.18851700 & 0.98379800 & 0.45197000 \\
\hline $\mathrm{C}$ & 4.90485300 & -0.44307900 & 2.48971300 \\
\hline $\mathrm{H}$ & 4.23900700 & -2.35444700 & 0.63997100 \\
\hline $\mathrm{H}$ & 4.52021100 & -1.37743200 & -0.81903600 \\
\hline $\mathrm{H}$ & 5.86840900 & -1.73635300 & 0.29205800 \\
\hline $\mathrm{H}$ & 4.91125700 & 1.87115600 & 1.02998800 \\
\hline $\mathrm{H}$ & 6.27510800 & 0.85618900 & 0.51216700 \\
\hline $\mathrm{H}$ & 4.90161100 & 1.13591600 & -0.58874800 \\
\hline $\mathrm{H}$ & 5.98428100 & -0.61154300 & 2.56083500 \\
\hline $\mathrm{H}$ & 4.64452500 & 0.44272600 & 3.07626000 \\
\hline $\mathrm{H}$ & 4.38266900 & -1.30455700 & 2.91629200 \\
\hline $\mathrm{H}$ & 1.21155700 & -0.76197000 & -2.75669900 \\
\hline \multicolumn{4}{|c|}{ TS3 } \\
\hline C & -0.36750100 & -0.97771400 & -1.04880400 \\
\hline $\mathrm{C}$ & 0.36640300 & -1.80846800 & -1.89701900 \\
\hline $\mathrm{C}$ & -0.10549500 & -3.05117700 & -2.30097600 \\
\hline $\mathrm{C}$ & -1.35491600 & -3.46040500 & -1.85457700 \\
\hline $\mathrm{C}$ & -2.11389100 & -2.65442200 & -1.01248700 \\
\hline $\mathrm{C}$ & -1.63131500 & -1.40813200 & -0.57691200 \\
\hline $\mathrm{H}$ & 0.48576600 & -3.67814500 & -2.96237500 \\
\hline $\mathrm{H}$ & -1.76290100 & -4.41983800 & -2.16290800 \\
\hline $\mathrm{H}$ & -3.08294400 & -2.99369400 & -0.68614400 \\
\hline $\mathrm{N}$ & -2.37404600 & -0.57243700 & 0.30198100 \\
\hline $\mathrm{C}$ & -3.48616000 & -1.00874600 & 0.95889700 \\
\hline $\mathrm{O}$ & -4.09412400 & -2.05736300 & 0.82123800 \\
\hline
\end{tabular}




\begin{tabular}{|c|c|c|c|}
\hline $\mathrm{O}$ & -3.90558600 & -0.08865900 & 1.87366100 \\
\hline $\mathrm{C}$ & 0.16818100 & 0.40026700 & -0.75131100 \\
\hline $\mathrm{H}$ & 0.73135600 & 0.79934500 & -1.59500200 \\
\hline $\mathrm{O}$ & 1.22560800 & 0.21665000 & 0.36898300 \\
\hline $\mathrm{C}$ & -0.87092100 & 1.29898900 & -0.31323900 \\
\hline $\mathrm{C}$ & -1.79792100 & 0.76214400 & 0.70510600 \\
\hline $\mathrm{H}$ & -1.32024800 & 0.58846800 & 1.68378200 \\
\hline $\mathrm{H}$ & -2.62895300 & 1.44506900 & 0.85672700 \\
\hline $\mathrm{C}$ & -5.07795000 & -0.46538600 & 2.57044800 \\
\hline $\mathrm{H}$ & -5.29206400 & 0.36003600 & 3.25035300 \\
\hline $\mathrm{H}$ & -5.91266000 & -0.61747700 & 1.88107000 \\
\hline $\mathrm{H}$ & -4.92081200 & -1.39064500 & 3.13101200 \\
\hline $\mathrm{C}$ & -0.90561900 & 2.60923900 & -0.80546200 \\
\hline $\mathrm{O}$ & -0.14258800 & 3.15323200 & -1.61008400 \\
\hline $\mathrm{O}$ & -1.98022300 & 3.35535600 & -0.29629200 \\
\hline $\mathrm{C}$ & -2.07016600 & 4.65204400 & -0.83084400 \\
\hline $\mathrm{H}$ & -2.22048100 & 4.63501000 & -1.91619700 \\
\hline $\mathrm{H}$ & -2.92971800 & 5.12471500 & -0.34620800 \\
\hline $\mathrm{H}$ & -1.16398300 & 5.23455300 & -0.63379000 \\
\hline $\mathrm{C}$ & 2.46618100 & 0.04802000 & 0.00746300 \\
\hline $\mathrm{O}$ & 2.90813100 & -0.06586100 & -1.12151900 \\
\hline $\mathrm{O}$ & 3.23568000 & 0.01806900 & 1.12567000 \\
\hline $\mathrm{C}$ & 4.66166300 & -0.15020100 & 1.00758300 \\
\hline $\mathrm{C}$ & 4.99969200 & -1.49807200 & 0.37077200 \\
\hline C & 5.28366500 & 1.01509900 & 0.23889400 \\
\hline $\mathrm{C}$ & 5.12531400 & -0.12712400 & 2.46213600 \\
\hline $\mathrm{H}$ & 4.49119100 & -2.30082600 & 0.91449100 \\
\hline $\mathrm{H}$ & 4.68537200 & -1.52219400 & -0.67259600 \\
\hline $\mathrm{H}$ & 6.08005300 & -1.67137000 & 0.42782700 \\
\hline $\mathrm{H}$ & 4.97990200 & 1.96222000 & 0.69586600 \\
\hline $\mathrm{H}$ & 6.37615500 & 0.94322300 & 0.28231600 \\
\hline $\mathrm{H}$ & 4.96227200 & 1.00672500 & -0.80265600 \\
\hline $\mathrm{H}$ & 6.21225600 & -0.24568300 & 2.51672600 \\
\hline $\mathrm{H}$ & 4.84766300 & 0.82163500 & 2.93022300 \\
\hline $\mathrm{H}$ & 4.65128500 & -0.93897500 & 3.02146200 \\
\hline $\mathrm{H}$ & 1.33148800 & -1.44349700 & -2.23615100 \\
\hline \multicolumn{4}{|l|}{2} \\
\hline $\mathrm{C}$ & 1.02852000 & -1.63670900 & 0.11007200 \\
\hline $\mathrm{C}$ & 1.89255100 & -2.73150100 & 0.20194400 \\
\hline C & 3.25742000 & -2.57789400 & 0.00949000 \\
\hline $\mathrm{C}$ & 3.76443700 & -1.31080900 & -0.27186200 \\
\hline $\mathrm{C}$ & 2.92662200 & -0.20392400 & -0.36035900 \\
\hline $\mathrm{C}$ & 1.55255500 & -0.35712900 & -0.16688900 \\
\hline $\mathrm{H}$ & 3.92094000 & -3.43343200 & 0.07837100 \\
\hline $\mathrm{H}$ & 4.83027100 & -1.17538700 & -0.42767700 \\
\hline $\mathrm{H}$ & 3.33562400 & 0.77645200 & -0.56047500 \\
\hline $\mathrm{N}$ & 0.63645600 & 0.72307700 & -0.23919400 \\
\hline $\mathrm{C}$ & 1.01660300 & 1.99499100 & 0.12864900 \\
\hline $\mathrm{O}$ & 2.09678400 & 2.30724000 & 0.57737700 \\
\hline $\mathrm{O}$ & 0.02337900 & 2.88477200 & -0.07819500 \\
\hline $\mathrm{C}$ & -0.40445900 & -1.78748100 & 0.31129700 \\
\hline $\mathrm{H}$ & -0.79226300 & -2.70243400 & 0.75201900 \\
\hline $\mathrm{C}$ & -1.25008200 & -0.81583900 & -0.05529500 \\
\hline $\mathrm{C}$ & -0.72195500 & 0.43328500 & -0.71275900 \\
\hline $\mathrm{H}$ & -0.71530600 & 0.31426000 & -1.80667400 \\
\hline $\mathrm{H}$ & -1.35678400 & 1.28289000 & -0.47954800 \\
\hline $\mathrm{C}$ & 0.34865200 & 4.21722000 & 0.31179300 \\
\hline $\mathrm{H}$ & -0.53079000 & 4.81291300 & 0.07193300 \\
\hline $\mathrm{H}$ & 0.56516900 & 4.26107000 & 1.38109100 \\
\hline $\mathrm{H}$ & 1.21984800 & 4.57316600 & -0.24146600 \\
\hline $\mathrm{C}$ & -2.70592600 & -1.01911100 & 0.13204200 \\
\hline
\end{tabular}




\begin{tabular}{|c|c|c|c|}
\hline $\mathrm{O}$ & -3.21514100 & -1.99784200 & 0.62542100 \\
\hline $\mathrm{O}$ & -3.41778900 & 0.02625400 & -0.33208500 \\
\hline $\mathrm{C}$ & -4.83135700 & -0.10895700 & -0.19712400 \\
\hline $\mathrm{H}$ & -5.10229600 & -0.22876900 & 0.85417300 \\
\hline $\mathrm{H}$ & -5.25756600 & 0.80672500 & -0.60383300 \\
\hline $\mathrm{H}$ & -5.18311100 & -0.98057700 & -0.75349200 \\
\hline $\mathrm{H}$ & 1.47174000 & -3.70866800 & 0.42380200 \\
\hline \multicolumn{4}{|c|}{ DABCO } \\
\hline $\mathrm{C}$ & -0.77901200 & 0.22600500 & -1.35786100 \\
\hline $\mathrm{C}$ & 0.77959400 & 0.22635000 & -1.35747300 \\
\hline $\mathrm{H}$ & -1.18076800 & -0.56275600 & -2.00384200 \\
\hline $\mathrm{H}$ & -1.18124100 & 1.18072700 & -1.71438500 \\
\hline $\mathrm{H}$ & 1.18204100 & -0.56181500 & -2.00374800 \\
\hline $\mathrm{H}$ & 1.18156800 & 1.18148000 & -1.71319500 \\
\hline $\mathrm{C}$ & -0.77940200 & -1.28881400 & 0.48314200 \\
\hline $\mathrm{H}$ & -1.18220400 & -1.45426700 & 1.48864900 \\
\hline $\mathrm{H}$ & -1.18166400 & -2.07438800 & -0.16628700 \\
\hline $\mathrm{C}$ & 0.77900900 & -1.28910100 & 0.48300000 \\
\hline $\mathrm{H}$ & 1.18194400 & -1.45535900 & 1.48832000 \\
\hline $\mathrm{H}$ & 1.18084400 & -2.07440000 & -0.16702700 \\
\hline $\mathrm{C}$ & 0.77925000 & 1.06268000 & 0.87495900 \\
\hline $\mathrm{H}$ & 1.18161500 & 0.89388000 & 1.88007100 \\
\hline $\mathrm{H}$ & 1.18163400 & 2.01605700 & 0.51487900 \\
\hline $\mathrm{C}$ & -0.77944100 & 1.06308500 & 0.87429800 \\
\hline $\mathrm{H}$ & -1.18275200 & 0.89510400 & 1.87916300 \\
\hline $\mathrm{H}$ & -1.18101200 & 2.01645800 & 0.51330100 \\
\hline $\mathrm{N}$ & 1.28509100 & -0.00023700 & 0.00052200 \\
\hline $\mathrm{N}$ & -1.28509000 & -0.00004200 & 0.00000800 \\
\hline \multicolumn{4}{|c|}{${ }^{t} \mathrm{BuOH}$} \\
\hline $\mathrm{C}$ & -0.68380700 & 1.25734600 & -0.51262900 \\
\hline $\mathrm{C}$ & 0.00539300 & -0.00002700 & 0.01742100 \\
\hline $\mathrm{H}$ & -1.74251300 & 1.26648700 & -0.22535300 \\
\hline $\mathrm{H}$ & -0.20692600 & 2.14907700 & -0.09574000 \\
\hline $\mathrm{H}$ & -0.63325500 & 1.30833700 & -1.60501000 \\
\hline $\mathrm{C}$ & 1.48483100 & 0.00125200 & -0.34615100 \\
\hline $\mathrm{C}$ & -0.68149100 & -1.25892000 & -0.51195400 \\
\hline $\mathrm{H}$ & 1.97218600 & -0.88496000 & 0.07035500 \\
\hline $\mathrm{H}$ & 1.61786000 & 0.00102900 & -1.43208300 \\
\hline $\mathrm{H}$ & 1.97025500 & 0.88878000 & 0.06977300 \\
\hline $\mathrm{H}$ & -0.63087000 & -1.31046000 & -1.60432100 \\
\hline $\mathrm{H}$ & -0.20287700 & -2.14949600 & -0.09455800 \\
\hline $\mathrm{H}$ & -1.74018000 & -1.26992200 & -0.22465900 \\
\hline $\mathrm{O}$ & -0.02483300 & 0.00038200 & 1.44332200 \\
\hline $\mathrm{H}$ & -0.95457300 & 0.00016300 & 1.71489900 \\
\hline \multicolumn{4}{|c|}{ INT3 } \\
\hline $\mathrm{C}$ & 0.96098266 & 0.69364161 & 0.00000000 \\
\hline $\mathrm{C}$ & 1.75792266 & 1.86415961 & 0.26705900 \\
\hline $\mathrm{C}$ & 3.10483966 & 1.84844761 & -0.20116000 \\
\hline $\mathrm{C}$ & 3.63254366 & 0.76562161 & -0.86692600 \\
\hline $\mathrm{C}$ & 2.86727866 & -0.38617039 & -1.11324700 \\
\hline $\mathrm{C}$ & 1.56646666 & -0.40320139 & -0.67227600 \\
\hline $\mathrm{H}$ & 3.71805066 & 2.71480461 & 0.00256300 \\
\hline $\mathrm{H}$ & 4.66740666 & 0.80553561 & -1.19814400 \\
\hline $\mathrm{H}$ & 3.29248666 & -1.24169539 & -1.62665800 \\
\hline $\mathrm{H}$ & 0.95962266 & -1.28864839 & -0.84656000 \\
\hline $\mathrm{N}$ & 1.18118566 & 2.91270261 & 0.90289000 \\
\hline $\mathrm{C}$ & 1.88458366 & 3.98727161 & 1.30056900 \\
\hline $\mathrm{O}$ & 3.08044866 & 4.21339161 & 1.40297500 \\
\hline $\mathrm{O}$ & 0.98197866 & 4.98755261 & 1.66700600 \\
\hline $\mathrm{C}$ & -0.42092734 & 0.44122161 & 0.35004500 \\
\hline $\mathrm{H}$ & -0.68162034 & -0.59386939 & 0.12935600 \\
\hline
\end{tabular}




\begin{tabular}{|c|c|c|c|}
\hline $\mathrm{C}$ & -1.52316034 & 1.08967361 & 0.84700900 \\
\hline $\mathrm{C}$ & -1.64274034 & 2.50965361 & 1.29255800 \\
\hline $\mathrm{H}$ & -2.39081934 & 2.62466261 & 2.07869400 \\
\hline $\mathrm{C}$ & 1.59534566 & 6.15765561 & 2.18038900 \\
\hline $\mathrm{H}$ & 0.78218566 & 6.84500061 & 2.42477200 \\
\hline $\mathrm{H}$ & 2.17910766 & 5.93361361 & 3.07695100 \\
\hline $\mathrm{H}$ & 2.26366166 & 6.60894761 & 1.44220000 \\
\hline $\mathrm{C}$ & -2.70647634 & 0.20557661 & 1.01164300 \\
\hline $\mathrm{O}$ & -2.79798434 & -0.95201939 & 0.66680400 \\
\hline $\mathrm{O}$ & -3.74834234 & 0.83239761 & 1.62596800 \\
\hline $\mathrm{C}$ & -4.88939234 & 0.00566761 & 1.83770700 \\
\hline $\mathrm{H}$ & -4.62786734 & -0.85294939 & 2.45934000 \\
\hline $\mathrm{H}$ & -5.62365834 & 0.63399861 & 2.34146500 \\
\hline $\mathrm{H}$ & -5.28131434 & -0.36037639 & 0.88591900 \\
\hline $\mathrm{H}$ & -0.66339234 & 2.88598361 & 1.59049800 \\
\hline $\mathrm{N}$ & -2.10271034 & 3.47627961 & 0.18832500 \\
\hline $\mathrm{C}$ & -1.14644534 & 3.48467661 & -0.97888400 \\
\hline $\mathrm{C}$ & -2.13117034 & 4.86254561 & 0.77324700 \\
\hline $\mathrm{C}$ & -3.47452734 & 3.14087561 & -0.31750900 \\
\hline $\mathrm{C}$ & -1.75414134 & 4.36420561 & -2.09558100 \\
\hline $\mathrm{H}$ & -0.20015534 & 3.85656661 & -0.58310500 \\
\hline $\mathrm{H}$ & -0.99800534 & 2.44610161 & -1.28043800 \\
\hline $\mathrm{H}$ & -2.92731234 & 4.86149661 & 1.52381000 \\
\hline $\mathrm{H}$ & -1.16439534 & 5.01365461 & 1.26067700 \\
\hline $\mathrm{C}$ & -2.39389034 & 5.87034161 & -0.37153600 \\
\hline $\mathrm{C}$ & -3.95922734 & 4.29891261 & -1.22532400 \\
\hline $\mathrm{H}$ & -4.11163334 & 2.98058361 & 0.55362800 \\
\hline $\mathrm{H}$ & -3.38306434 & 2.19144661 & -0.85161100 \\
\hline $\mathrm{H}$ & -0.98484934 & 5.02023661 & -2.51141300 \\
\hline $\mathrm{H}$ & -2.14519934 & 3.75170261 & -2.91383500 \\
\hline $\mathrm{N}$ & -2.85235934 & 5.18368461 & -1.57896400 \\
\hline $\mathrm{H}$ & -3.14779834 & 6.60206061 & -0.06742800 \\
\hline $\mathrm{H}$ & -1.47690734 & 6.41438561 & -0.61566900 \\
\hline $\mathrm{H}$ & -4.72437034 & 4.89330461 & -0.71672200 \\
\hline $\mathrm{H}$ & -4.40319634 & 3.89846161 & -2.14081500 \\
\hline \multicolumn{4}{|c|}{ TS4 } \\
\hline $\mathrm{C}$ & -1.17616700 & -1.37053100 & 1.07673500 \\
\hline $\mathrm{C}$ & -1.52614500 & -2.01393300 & -0.13759900 \\
\hline $\mathrm{C}$ & -1.10064800 & -3.35095200 & -0.29709100 \\
\hline $\mathrm{C}$ & -0.43025400 & -4.03035600 & 0.70973700 \\
\hline $\mathrm{C}$ & -0.16858000 & -3.40793300 & 1.93401700 \\
\hline $\mathrm{C}$ & -0.55265400 & -2.08229600 & 2.10382300 \\
\hline $\mathrm{H}$ & -1.34586600 & -3.83639500 & -1.23677700 \\
\hline $\mathrm{H}$ & -0.13089100 & -5.06303900 & 0.55170200 \\
\hline $\mathrm{H}$ & 0.32043100 & -3.94735200 & 2.73875700 \\
\hline $\mathrm{H}$ & -0.32937800 & -1.56679900 & 3.03681400 \\
\hline $\mathrm{N}$ & -2.19790700 & -1.41606300 & -1.17866200 \\
\hline $\mathrm{C}$ & -2.97049300 & -0.39227900 & -0.85341500 \\
\hline $\mathrm{O}$ & -3.12584700 & 0.16712200 & 0.26580100 \\
\hline $\mathrm{O}$ & -3.67557600 & 0.09049600 & -1.91000600 \\
\hline $\mathrm{C}$ & -1.32093300 & 0.09485200 & 1.22739600 \\
\hline $\mathrm{H}$ & -1.80751400 & 0.46573700 & 2.12498600 \\
\hline $\mathrm{C}$ & -0.48415400 & 0.99206500 & 0.59197000 \\
\hline $\mathrm{C}$ & 0.20435000 & 0.66719900 & -0.68970700 \\
\hline $\mathrm{H}$ & 0.22801900 & 1.53710500 & -1.35057400 \\
\hline $\mathrm{C}$ & -4.51752300 & 1.19287300 & -1.62217200 \\
\hline $\mathrm{H}$ & -4.97631200 & 1.46994300 & -2.57238000 \\
\hline $\mathrm{H}$ & -5.29002900 & 0.92258400 & -0.89726600 \\
\hline $\mathrm{H}$ & -3.94729500 & 2.03345400 & -1.21651700 \\
\hline $\mathrm{C}$ & -0.56815200 & 2.38300100 & 1.03946100 \\
\hline $\mathrm{O}$ & -1.17896400 & 2.79835500 & 1.99962500 \\
\hline
\end{tabular}




\begin{tabular}{|c|c|c|c|}
\hline $\mathrm{O}$ & 0.16673700 & 3.23254500 & 0.25237800 \\
\hline $\mathrm{C}$ & 0.08649200 & 4.60043600 & 0.63773600 \\
\hline $\mathrm{H}$ & 0.45833100 & 4.73811300 & 1.65579000 \\
\hline $\mathrm{H}$ & 0.70322400 & 5.14995100 & -0.07372500 \\
\hline $\mathrm{H}$ & -0.94721300 & 4.95014000 & 0.59678700 \\
\hline $\mathrm{H}$ & -0.27772300 & -0.16219800 & -1.21322900 \\
\hline $\mathrm{N}$ & 4.15889400 & -0.44751000 & -0.49716800 \\
\hline $\mathrm{C}$ & 3.72493400 & -0.31039600 & -1.88495600 \\
\hline $\mathrm{C}$ & 3.97111900 & 0.83093900 & 0.18552000 \\
\hline $\mathrm{C}$ & 3.32567700 & -1.45634700 & 0.15878200 \\
\hline $\mathrm{C}$ & 2.19936900 & -0.04506200 & -1.96247000 \\
\hline $\mathrm{H}$ & 4.28397600 & 0.51245000 & -2.33949000 \\
\hline $\mathrm{H}$ & 3.97610800 & -1.22499400 & -2.42814000 \\
\hline $\mathrm{H}$ & 4.21159000 & 0.68973000 & 1.24282000 \\
\hline $\mathrm{H}$ & 4.67818500 & 1.55852200 & -0.22209000 \\
\hline $\mathrm{C}$ & 2.51864200 & 1.34551700 & 0.01950700 \\
\hline $\mathrm{C}$ & 1.85271900 & -0.98969400 & 0.25540000 \\
\hline $\mathrm{H}$ & 3.72563800 & -1.65215500 & 1.15694700 \\
\hline $\mathrm{H}$ & 3.39711700 & -2.38483600 & -0.41450200 \\
\hline $\mathrm{H}$ & 1.95025300 & 0.81381200 & -2.59016000 \\
\hline $\mathrm{H}$ & 1.63343600 & -0.90958000 & -2.31945400 \\
\hline $\mathrm{N}$ & 1.68979800 & 0.25054700 & -0.58273800 \\
\hline $\mathrm{H}$ & 2.04467600 & 1.61059200 & 0.96553500 \\
\hline $\mathrm{H}$ & 2.43625100 & 2.20488100 & -0.64989100 \\
\hline $\mathrm{H}$ & 1.54991700 & -0.71260200 & 1.26558400 \\
\hline $\mathrm{H}$ & 1.14260700 & -1.73398800 & -0.11481700 \\
\hline \multicolumn{4}{|c|}{ INT4 } \\
\hline $\mathrm{C}$ & 1.22923200 & -1.41567700 & -0.98712100 \\
\hline $\mathrm{C}$ & 1.52462200 & -2.12263500 & 0.19008500 \\
\hline $\mathrm{C}$ & 1.06197800 & -3.43796300 & 0.32659700 \\
\hline $\mathrm{C}$ & 0.35723200 & -4.04598000 & -0.70698100 \\
\hline $\mathrm{C}$ & 0.12027000 & -3.35954500 & -1.89987500 \\
\hline $\mathrm{C}$ & 0.56551300 & -2.04674000 & -2.03406400 \\
\hline $\mathrm{H}$ & 1.28726000 & -3.96673300 & 1.24763800 \\
\hline $\mathrm{H}$ & 0.00915300 & -5.06844900 & -0.59179200 \\
\hline $\mathrm{H}$ & -0.40403100 & -3.84556300 & -2.71687700 \\
\hline $\mathrm{H}$ & 0.36763300 & -1.48822200 & -2.94723300 \\
\hline $\mathrm{N}$ & 2.25831000 & -1.54336300 & 1.23146200 \\
\hline $\mathrm{C}$ & 2.91079200 & -0.48358500 & 0.90500700 \\
\hline $\mathrm{O}$ & 2.88037600 & 0.15882700 & -0.25176800 \\
\hline $\mathrm{O}$ & 3.74578500 & 0.04472200 & 1.80231500 \\
\hline $\mathrm{C}$ & 1.58540700 & 0.04457300 & -1.00993600 \\
\hline $\mathrm{H}$ & 1.88974200 & 0.37900000 & -2.00211600 \\
\hline $\mathrm{C}$ & 0.56091600 & 0.97872900 & -0.48210100 \\
\hline $\mathrm{C}$ & -0.17324400 & 0.70914900 & 0.74739800 \\
\hline $\mathrm{H}$ & -0.27898300 & 1.58734900 & 1.39208800 \\
\hline $\mathrm{C}$ & 4.33808400 & 1.30162600 & 1.48250700 \\
\hline $\mathrm{H}$ & 4.88092000 & 1.59689800 & 2.37995800 \\
\hline $\mathrm{H}$ & 5.02661600 & 1.20636000 & 0.64002500 \\
\hline $\mathrm{H}$ & 3.57365400 & 2.04171700 & 1.23419600 \\
\hline $\mathrm{C}$ & 0.67279900 & 2.31740100 & -0.97142200 \\
\hline $\mathrm{O}$ & 1.39612000 & 2.72320200 & -1.87013600 \\
\hline $\mathrm{O}$ & -0.17064200 & 3.20810900 & -0.31827500 \\
\hline $\mathrm{C}$ & -0.04218800 & 4.55042500 & -0.75631100 \\
\hline $\mathrm{H}$ & -0.27898300 & 4.64137500 & -1.81967100 \\
\hline $\mathrm{H}$ & -0.74679900 & 5.13280300 & -0.15951100 \\
\hline $\mathrm{H}$ & 0.97569100 & 4.91828900 & -0.60272400 \\
\hline $\mathrm{H}$ & 0.21570100 & -0.13290400 & 1.32722200 \\
\hline $\mathrm{N}$ & -4.19277300 & -0.38356500 & 0.39065900 \\
\hline $\mathrm{C}$ & -3.82590800 & -0.21870600 & 1.79571800 \\
\hline $\mathrm{C}$ & -3.94769700 & 0.87730600 & -0.31071500 \\
\hline
\end{tabular}




\begin{tabular}{|c|c|c|c|}
\hline $\mathrm{C}$ & -3.33903000 & -1.41557900 & -0.20011500 \\
\hline $\mathrm{C}$ & -2.30232800 & 0.03651700 & 1.94291900 \\
\hline $\mathrm{H}$ & -4.39976400 & 0.61942900 & 2.20222400 \\
\hline $\mathrm{H}$ & -4.11487500 & -1.11859000 & 2.34546100 \\
\hline $\mathrm{H}$ & -4.14022700 & 0.71481100 & -1.37518000 \\
\hline $\mathrm{H}$ & -4.66334900 & 1.62365800 & 0.04555400 \\
\hline $\mathrm{C}$ & -2.49654300 & 1.36975900 & -0.08231700 \\
\hline C & -1.85677400 & -0.96632600 & -0.22026900 \\
\hline $\mathrm{H}$ & -3.68802600 & -1.62298200 & -1.21543400 \\
\hline $\mathrm{H}$ & -3.45636800 & -2.33308100 & 0.38423900 \\
\hline $\mathrm{H}$ & -2.07749400 & 0.91233000 & 2.55764400 \\
\hline $\mathrm{H}$ & -1.76981400 & -0.82298300 & 2.36052700 \\
\hline $\mathrm{N}$ & -1.72467100 & 0.28260900 & 0.59079700 \\
\hline $\mathrm{H}$ & -1.95885200 & 1.59937500 & -1.00390400 \\
\hline $\mathrm{H}$ & -2.43535500 & 2.24842800 & 0.56420100 \\
\hline $\mathrm{H}$ & -1.48944800 & -0.71398500 & -1.21567800 \\
\hline $\mathrm{H}$ & -1.18198100 & -1.71346900 & 0.20761400 \\
\hline \multicolumn{4}{|c|}{ TS5 } \\
\hline C & 1.22213900 & -1.36481800 & -1.00420300 \\
\hline $\mathrm{C}$ & 1.54798600 & -2.11614700 & 0.13563300 \\
\hline $\mathrm{C}$ & 1.08910000 & -3.43478700 & 0.23529900 \\
\hline $\mathrm{C}$ & 0.35755900 & -4.00192700 & -0.80329200 \\
\hline $\mathrm{C}$ & 0.08920600 & -3.26997100 & -1.96158600 \\
\hline $\mathrm{C}$ & 0.53087000 & -1.95243400 & -2.05744700 \\
\hline $\mathrm{H}$ & 1.34061600 & -3.99931300 & 1.12762500 \\
\hline $\mathrm{H}$ & 0.01367800 & -5.02868000 & -0.71952500 \\
\hline $\mathrm{H}$ & -0.45749900 & -3.72375900 & -2.78219300 \\
\hline $\mathrm{H}$ & 0.31520200 & -1.36215900 & -2.94603900 \\
\hline N & 2.32599700 & -1.57721500 & 1.16821100 \\
\hline $\mathrm{C}$ & 2.98718400 & -0.52426400 & 0.85151500 \\
\hline $\mathrm{O}$ & 2.93082500 & 0.15167100 & -0.29430900 \\
\hline $\mathrm{O}$ & 3.86597000 & -0.03396900 & 1.72546000 \\
\hline $\mathrm{C}$ & 1.62349500 & 0.08396100 & -0.98730900 \\
\hline $\mathrm{H}$ & 1.87067800 & 0.44873600 & -1.98479700 \\
\hline $\mathrm{C}$ & 0.65188100 & 1.02933900 & -0.35084400 \\
\hline $\mathrm{C}$ & -0.05482200 & 0.73252300 & 0.82233500 \\
\hline $\mathrm{H}$ & -0.31106000 & 1.57178300 & 1.46814200 \\
\hline $\mathrm{C}$ & 4.46101600 & 1.22691100 & 1.42573600 \\
\hline $\mathrm{H}$ & 5.04374500 & 1.48314900 & 2.30969500 \\
\hline $\mathrm{H}$ & 5.11266500 & 1.15374000 & 0.55256700 \\
\hline $\mathrm{H}$ & 3.69662400 & 1.98432900 & 1.23792100 \\
\hline $\mathrm{C}$ & 0.69341600 & 2.37970200 & -0.86825600 \\
\hline $\mathrm{O}$ & 1.38219400 & 2.77802000 & -1.79148400 \\
\hline $\mathrm{O}$ & -0.15319500 & 3.23943600 & -0.21066400 \\
\hline $\mathrm{C}$ & -0.09331500 & 4.58135200 & -0.67125300 \\
\hline $\mathrm{H}$ & -0.36067200 & 4.64287300 & -1.72907700 \\
\hline $\mathrm{H}$ & -0.80769900 & 5.13942000 & -0.06482200 \\
\hline $\mathrm{H}$ & 0.91181900 & 4.99189300 & -0.54709200 \\
\hline $\mathrm{H}$ & 0.21526200 & -0.17235200 & 1.36633600 \\
\hline $\mathrm{N}$ & -4.30652500 & -0.41061300 & 0.36209500 \\
\hline $\mathrm{C}$ & -3.95818400 & -0.28778000 & 1.77872200 \\
\hline $\mathrm{C}$ & -4.04532400 & 0.87012300 & -0.30075500 \\
\hline $\mathrm{C}$ & -3.44708500 & -1.43078000 & -0.24558000 \\
\hline $\mathrm{C}$ & -2.43302000 & -0.02363500 & 1.94598900 \\
\hline $\mathrm{H}$ & -4.54527100 & 0.53261900 & 2.20281000 \\
\hline $\mathrm{H}$ & -4.25066100 & -1.20786800 & 2.29286000 \\
\hline $\mathrm{H}$ & -4.23324300 & 0.73988400 & -1.37084700 \\
\hline $\mathrm{H}$ & -4.75869000 & 1.61026100 & 0.07382000 \\
\hline $\mathrm{C}$ & -2.58437300 & 1.33322700 & -0.04242800 \\
\hline $\mathrm{C}$ & -1.96003300 & -0.98218600 & -0.21381600 \\
\hline $\mathrm{H}$ & -3.78023800 & -1.60232300 & -1.27326100 \\
\hline
\end{tabular}




\begin{tabular}{|c|c|c|c|}
\hline $\mathrm{H}$ & -3.58453100 & -2.36475300 & 0.30788400 \\
\hline $\mathrm{H}$ & -2.22793800 & 0.84663900 & 2.57817100 \\
\hline $\mathrm{H}$ & -1.90686800 & -0.88488300 & 2.37026900 \\
\hline $\mathrm{N}$ & -1.85162600 & 0.23425200 & 0.61605900 \\
\hline $\mathrm{H}$ & -2.03885100 & 1.57677900 & -0.95870200 \\
\hline $\mathrm{H}$ & -2.52811700 & 2.20536100 & 0.61552800 \\
\hline $\mathrm{H}$ & -1.57729000 & -0.71855100 & -1.20190000 \\
\hline $\mathrm{H}$ & -1.29690300 & -1.74684300 & 0.20497900 \\
\hline \multicolumn{4}{|l|}{3} \\
\hline $\mathrm{C}$ & -1.55049100 & -0.49544800 & 0.50346000 \\
\hline $\mathrm{C}$ & -2.09835200 & 0.47241400 & -0.34948600 \\
\hline $\mathrm{C}$ & -3.42899100 & 0.34380800 & -0.75553500 \\
\hline $\mathrm{C}$ & -4.19302200 & -0.72844800 & -0.31010300 \\
\hline $\mathrm{C}$ & -3.64514100 & -1.68403100 & 0.54633600 \\
\hline $\mathrm{C}$ & -2.31872400 & -1.56293400 & 0.95194400 \\
\hline $\mathrm{H}$ & -3.83993100 & 1.10052500 & -1.41565300 \\
\hline $\mathrm{H}$ & -5.22642200 & -0.81958700 & -0.63074700 \\
\hline $\mathrm{H}$ & -4.24614200 & -2.51887200 & 0.89167000 \\
\hline $\mathrm{H}$ & -1.87477100 & -2.30517000 & 1.61161800 \\
\hline $\mathrm{N}$ & -1.34525000 & 1.56885400 & -0.79222500 \\
\hline $\mathrm{C}$ & -0.27909100 & 1.80621500 & -0.13467600 \\
\hline $\mathrm{O}$ & 0.23710000 & 1.06974600 & 0.86884400 \\
\hline $\mathrm{O}$ & 0.42773600 & 2.90273200 & -0.39198100 \\
\hline $\mathrm{C}$ & -0.09205000 & -0.33598200 & 0.82165400 \\
\hline $\mathrm{H}$ & 0.15474600 & -0.69673400 & 1.82287600 \\
\hline $\mathrm{C}$ & 0.82999400 & -0.99534100 & -0.18942200 \\
\hline $\mathrm{C}$ & 0.41815100 & -1.55606300 & -1.32598500 \\
\hline $\mathrm{H}$ & 1.12994000 & -1.99056100 & -2.01885100 \\
\hline $\mathrm{C}$ & 1.64435800 & 3.10560300 & 0.32607100 \\
\hline $\mathrm{H}$ & 2.10761300 & 3.97290400 & -0.14219300 \\
\hline $\mathrm{H}$ & 1.44150700 & 3.31051000 & 1.37981300 \\
\hline $\mathrm{H}$ & 2.29812700 & 2.23363400 & 0.25459200 \\
\hline $\mathrm{C}$ & 2.27537700 & -0.94023900 & 0.18273300 \\
\hline $\mathrm{O}$ & 2.67924600 & -0.49416200 & 1.23204400 \\
\hline $\mathrm{O}$ & 3.08464600 & -1.43838300 & -0.76407800 \\
\hline $\mathrm{C}$ & 4.47456400 & -1.39891000 & -0.44397000 \\
\hline $\mathrm{H}$ & 4.67324000 & -1.97096100 & 0.46489200 \\
\hline $\mathrm{H}$ & 4.98687800 & -1.84001700 & -1.29738400 \\
\hline $\mathrm{H}$ & 4.79915700 & -0.36715400 & -0.29017900 \\
\hline $\mathrm{H}$ & -0.63651300 & -1.59384300 & -1.58162300 \\
\hline \multicolumn{4}{|c|}{ TS4, } \\
\hline $\mathrm{C}$ & 2.18412000 & -1.27155800 & 0.68307800 \\
\hline $\mathrm{C}$ & 2.57363600 & -0.48392800 & -0.39940200 \\
\hline $\mathrm{C}$ & 3.67880100 & 0.35364100 & -0.34671300 \\
\hline $\mathrm{C}$ & 4.39639800 & 0.33753100 & 0.85828200 \\
\hline $\mathrm{C}$ & 4.01873800 & -0.45805500 & 1.94096400 \\
\hline $\mathrm{C}$ & 2.88252900 & -1.28239400 & 1.87237400 \\
\hline $\mathrm{H}$ & 3.95457700 & 0.99329300 & -1.17671500 \\
\hline $\mathrm{H}$ & 5.27244600 & 0.97267900 & 0.95560100 \\
\hline $\mathrm{H}$ & 4.60928300 & -0.43343400 & 2.85138000 \\
\hline $\mathrm{H}$ & 2.57489300 & -1.88798400 & 2.72050700 \\
\hline $\mathrm{N}$ & 1.55719100 & -0.79963700 & -1.30006500 \\
\hline $\mathrm{C}$ & 1.03516400 & 0.08397400 & -2.16570700 \\
\hline $\mathrm{O}$ & 1.42883900 & 1.22362300 & -2.40061500 \\
\hline $\mathrm{O}$ & -0.07755900 & -0.42902300 & -2.76535400 \\
\hline $\mathrm{C}$ & 0.92046400 & -1.84728700 & 0.09275600 \\
\hline $\mathrm{H}$ & 0.99908500 & -2.81389100 & -0.40059300 \\
\hline $\mathrm{C}$ & -0.35531200 & -1.59174300 & 0.64278100 \\
\hline $\mathrm{C}$ & -0.61183000 & -0.53552500 & 1.63230700 \\
\hline $\mathrm{H}$ & -1.46264500 & -0.78691100 & 2.26867000 \\
\hline $\mathrm{C}$ & -0.66020700 & 0.42115800 & -3.73886500 \\
\hline
\end{tabular}




\begin{tabular}{|c|c|c|c|}
\hline $\mathrm{H}$ & -1.51952800 & -0.12216800 & -4.13182300 \\
\hline $\mathrm{H}$ & -0.98046100 & 1.37095000 & -3.29645200 \\
\hline $\mathrm{H}$ & 0.04964600 & 0.64337900 & -4.53920900 \\
\hline $\mathrm{C}$ & -1.42235200 & -2.47466400 & 0.22848600 \\
\hline $\mathrm{O}$ & -1.33645500 & -3.42672500 & -0.52224100 \\
\hline $\mathrm{O}$ & -2.63596200 & -2.15776600 & 0.81040500 \\
\hline $\mathrm{C}$ & -3.68321900 & -3.06374300 & 0.49078600 \\
\hline $\mathrm{H}$ & -3.43897100 & -4.07346000 & 0.82914200 \\
\hline $\mathrm{H}$ & -4.56941300 & -2.69476400 & 1.00872700 \\
\hline $\mathrm{H}$ & -3.85447500 & -3.09507700 & -0.58778200 \\
\hline $\mathrm{H}$ & 0.26233100 & -0.30725100 & 2.25054700 \\
\hline $\mathrm{N}$ & -1.00201800 & 0.88329900 & 1.06459000 \\
\hline $\mathrm{C}$ & 0.22706100 & 1.64982400 & 0.66821700 \\
\hline $\mathrm{C}$ & -1.71243700 & 1.66728000 & 2.12117600 \\
\hline $\mathrm{C}$ & -1.90851100 & 0.76199500 & -0.12776600 \\
\hline $\mathrm{C}$ & -0.21128400 & 2.94028800 & -0.05958600 \\
\hline $\mathrm{H}$ & 0.78396700 & 1.84197200 & 1.59056600 \\
\hline $\mathrm{H}$ & 0.82937000 & 1.00988700 & 0.03029100 \\
\hline $\mathrm{H}$ & -2.66813700 & 1.16464900 & 2.29389300 \\
\hline $\mathrm{H}$ & -1.11443700 & 1.59998900 & 3.03438500 \\
\hline $\mathrm{C}$ & -1.88729500 & 3.12504200 & 1.61898000 \\
\hline $\mathrm{C}$ & -2.41929000 & 2.17832100 & -0.49123100 \\
\hline $\mathrm{H}$ & -2.70439700 & 0.06826100 & 0.15017800 \\
\hline $\mathrm{H}$ & -1.31863500 & 0.29540200 & -0.92090000 \\
\hline $\mathrm{H}$ & 0.38185700 & 3.79035500 & 0.28788900 \\
\hline $\mathrm{H}$ & -0.04281200 & 2.82827100 & -1.13484900 \\
\hline $\mathrm{N}$ & -1.63128700 & 3.21040300 & 0.18352100 \\
\hline $\mathrm{H}$ & -2.90326400 & 3.47252900 & 1.82624300 \\
\hline $\mathrm{H}$ & -1.19299700 & 3.79739000 & 2.13114900 \\
\hline $\mathrm{H}$ & -3.46684000 & 2.30323700 & -0.19924900 \\
\hline $\mathrm{H}$ & -2.35492800 & 2.33395400 & -1.57117200 \\
\hline \multicolumn{4}{|c|}{ INT4, } \\
\hline $\mathrm{C}$ & -2.31885700 & -1.00649800 & -0.87352400 \\
\hline $\mathrm{C}$ & -2.58916400 & -0.47015600 & 0.38412500 \\
\hline $\mathrm{C}$ & -3.61802500 & 0.41293300 & 0.64705500 \\
\hline $\mathrm{C}$ & -4.40897900 & 0.72657600 & -0.47143700 \\
\hline $\mathrm{C}$ & -4.16206300 & 0.19149100 & -1.73660300 \\
\hline $\mathrm{C}$ & -3.09318400 & -0.69473000 & -1.96911800 \\
\hline $\mathrm{H}$ & -3.79329600 & 0.84066000 & 1.62708400 \\
\hline $\mathrm{H}$ & -5.23997100 & 1.41519000 & -0.34832700 \\
\hline $\mathrm{H}$ & -4.80881400 & 0.47190200 & -2.56224200 \\
\hline $\mathrm{H}$ & -2.89967100 & -1.09231600 & -2.96121600 \\
\hline $\mathrm{N}$ & -1.51279600 & -1.10951600 & 1.01829100 \\
\hline $\mathrm{C}$ & -0.80723900 & -0.65561500 & 2.08256900 \\
\hline $\mathrm{O}$ & -1.10752700 & 0.31121800 & 2.76506400 \\
\hline $\mathrm{O}$ & 0.29296000 & -1.40693700 & 2.28735700 \\
\hline $\mathrm{C}$ & -1.06785500 & -1.72269400 & -0.36331100 \\
\hline $\mathrm{H}$ & -1.15019400 & -2.80735600 & -0.26951900 \\
\hline $\mathrm{C}$ & 0.24713900 & -1.35049000 & -0.91239900 \\
\hline $\mathrm{C}$ & 0.47626300 & -0.15490000 & -1.68207100 \\
\hline $\mathrm{H}$ & 1.31089700 & -0.25131600 & -2.37987600 \\
\hline $\mathrm{C}$ & 1.07031600 & -1.02097800 & 3.41283900 \\
\hline $\mathrm{H}$ & 1.90940600 & -1.71474800 & 3.43928700 \\
\hline $\mathrm{H}$ & 1.42714200 & 0.00889300 & 3.30685500 \\
\hline $\mathrm{H}$ & 0.48254900 & -1.09017800 & 4.33091700 \\
\hline $\mathrm{C}$ & 1.28300400 & -2.32272400 & -0.74711400 \\
\hline $\mathrm{O}$ & 1.19023800 & -3.42056800 & -0.22078300 \\
\hline $\mathrm{O}$ & 2.50029000 & -1.92130900 & -1.28542100 \\
\hline $\mathrm{C}$ & 3.50865100 & -2.91663600 & -1.22800400 \\
\hline $\mathrm{H}$ & 3.21357500 & -3.80739400 & -1.78871500 \\
\hline $\mathrm{H}$ & 4.39989600 & -2.47037000 & -1.67307600 \\
\hline
\end{tabular}




\begin{tabular}{|c|c|c|c|}
\hline $\mathrm{H}$ & 3.70694000 & -3.21469000 & -0.19517500 \\
\hline $\mathrm{H}$ & -0.41076100 & 0.24807200 & -2.18388600 \\
\hline $\mathrm{N}$ & 0.95474800 & 1.19155100 & -0.85019000 \\
\hline $\mathrm{C}$ & -0.21233300 & 1.83117700 & -0.17636100 \\
\hline $\mathrm{C}$ & 1.55482500 & 2.17731000 & -1.78823500 \\
\hline $\mathrm{C}$ & 1.97561900 & 0.82576600 & 0.17718500 \\
\hline $\mathrm{C}$ & 0.30322600 & 2.97015000 & 0.73545200 \\
\hline $\mathrm{H}$ & -0.88423900 & 2.18177500 & -0.96648700 \\
\hline $\mathrm{H}$ & -0.72995400 & 1.06512700 & 0.39520700 \\
\hline $\mathrm{H}$ & 2.47130700 & 1.72646800 & -2.17957200 \\
\hline $\mathrm{H}$ & 0.85364000 & 2.30973000 & -2.61797800 \\
\hline $\mathrm{C}$ & 1.82734700 & 3.49522600 & -1.01313300 \\
\hline $\mathrm{C}$ & 2.52427900 & 2.13160600 & 0.80558100 \\
\hline $\mathrm{H}$ & 2.73491200 & 0.22452200 & -0.32804500 \\
\hline $\mathrm{H}$ & 1.47055000 & 0.17325600 & 0.89341400 \\
\hline $\mathrm{H}$ & -0.30186100 & 3.87155000 & 0.60376800 \\
\hline $\mathrm{H}$ & 0.23298300 & 2.66588500 & 1.78426900 \\
\hline $\mathrm{N}$ & 1.70134300 & 3.28254600 & 0.42784800 \\
\hline $\mathrm{H}$ & 2.83353800 & 3.86293300 & -1.23372800 \\
\hline $\mathrm{H}$ & 1.11587000 & 4.27289400 & -1.30660300 \\
\hline $\mathrm{H}$ & 3.55003600 & 2.32340000 & 0.47484400 \\
\hline $\mathrm{H}$ & 2.53694700 & 2.05391800 & 1.89645300 \\
\hline \multicolumn{4}{|c|}{ TS5, } \\
\hline $\mathrm{C}$ & 2.34699100 & 1.01227600 & -0.74236800 \\
\hline $\mathrm{C}$ & 2.47984400 & 0.52019400 & 0.55485800 \\
\hline $\mathrm{C}$ & 3.45368000 & -0.37480200 & 0.95103400 \\
\hline $\mathrm{C}$ & 4.33677400 & -0.75502800 & -0.07411100 \\
\hline $\mathrm{C}$ & 4.22593700 & -0.26814800 & -1.37785800 \\
\hline $\mathrm{C}$ & 3.21054000 & 0.63537100 & -1.74649100 \\
\hline $\mathrm{H}$ & 3.52400500 & -0.76486400 & 1.95945700 \\
\hline $\mathrm{H}$ & 5.13381100 & -1.45632200 & 0.15513700 \\
\hline $\mathrm{H}$ & 4.93990600 & -0.59910200 & -2.12553800 \\
\hline $\mathrm{H}$ & 3.12271600 & 0.99425700 & -2.76768000 \\
\hline $\mathrm{N}$ & 1.36538700 & 1.21289600 & 1.05288300 \\
\hline $\mathrm{C}$ & 0.53190500 & 0.82873300 & 2.05280900 \\
\hline $\mathrm{O}$ & 0.71804200 & -0.11906100 & 2.79919300 \\
\hline $\mathrm{O}$ & -0.54459000 & 1.63321400 & 2.10885000 \\
\hline $\mathrm{C}$ & 1.07030300 & 1.76857100 & -0.37542600 \\
\hline $\mathrm{H}$ & 1.15331300 & 2.85636500 & -0.33207200 \\
\hline $\mathrm{C}$ & -0.19862700 & 1.35700200 & -1.01477200 \\
\hline $\mathrm{C}$ & -0.34354100 & 0.16752200 & -1.76862900 \\
\hline $\mathrm{H}$ & -1.18413900 & 0.17222400 & -2.46199900 \\
\hline $\mathrm{C}$ & -1.46627400 & 1.33119300 & 3.14770800 \\
\hline $\mathrm{H}$ & -2.24703100 & 2.08625400 & 3.06980700 \\
\hline $\mathrm{H}$ & -1.88960400 & 0.33040400 & 3.01585300 \\
\hline $\mathrm{H}$ & -0.97700700 & 1.37790600 & 4.12286600 \\
\hline $\mathrm{C}$ & -1.29863500 & 2.27340800 & -0.86895300 \\
\hline $\mathrm{O}$ & -1.26288000 & 3.38619000 & -0.37356300 \\
\hline $\mathrm{O}$ & -2.48432500 & 1.77651500 & -1.37553800 \\
\hline $\mathrm{C}$ & -3.56563900 & 2.69274100 & -1.31424000 \\
\hline $\mathrm{H}$ & -3.34457400 & 3.59944300 & -1.88305900 \\
\hline $\mathrm{H}$ & -4.42432500 & 2.17619400 & -1.74586000 \\
\hline $\mathrm{H}$ & -3.77447700 & 2.98097700 & -0.28063200 \\
\hline $\mathrm{H}$ & 0.56634400 & -0.24685000 & -2.20937500 \\
\hline $\mathrm{N}$ & -0.81163400 & -1.28523400 & -0.87631300 \\
\hline $\mathrm{C}$ & 0.36471100 & -1.85500600 & -0.16551000 \\
\hline $\mathrm{C}$ & -1.34910500 & -2.30645600 & -1.80444200 \\
\hline $\mathrm{C}$ & -1.86162100 & -0.94813300 & 0.11552000 \\
\hline $\mathrm{C}$ & -0.02838600 & -3.23033600 & 0.42913600 \\
\hline $\mathrm{H}$ & 1.19860300 & -1.91318600 & -0.87302500 \\
\hline $\mathrm{H}$ & 0.63519800 & -1.15740600 & 0.62551600 \\
\hline
\end{tabular}




\begin{tabular}{|c|c|c|c|}
\hline $\mathrm{H}$ & -2.10722000 & -1.82687100 & -2.43044800 \\
\hline $\mathrm{H}$ & -0.52370000 & -2.62833700 & -2.44784700 \\
\hline $\mathrm{C}$ & -1.93565400 & -3.47345300 & -0.95959600 \\
\hline $\mathrm{C}$ & -2.06482000 & -2.17491400 & 1.03812400 \\
\hline $\mathrm{H}$ & -2.75834800 & -0.68048200 & -0.45031100 \\
\hline $\mathrm{H}$ & -1.52951000 & -0.04977800 & 0.64416600 \\
\hline $\mathrm{H}$ & 0.39899500 & -4.05488600 & -0.15029300 \\
\hline $\mathrm{H}$ & 0.34118000 & -3.30945100 & 1.45511400 \\
\hline $\mathrm{N}$ & -1.48594800 & -3.38009700 & 0.42970800 \\
\hline $\mathrm{H}$ & -3.02957000 & -3.44320800 & -0.96707900 \\
\hline $\mathrm{H}$ & -1.62529200 & -4.43866100 & -1.37001600 \\
\hline $\mathrm{H}$ & -3.12946400 & -2.34343300 & 1.22530400 \\
\hline $\mathrm{H}$ & -1.56868100 & -2.01681000 & 2.00154700 \\
\hline \multicolumn{4}{|l|}{3 , } \\
\hline $\mathrm{C}$ & 0.06239200 & -1.34284400 & -0.31065100 \\
\hline $\mathrm{C}$ & 1.34614700 & -1.17511500 & 0.19856800 \\
\hline $\mathrm{C}$ & 2.18857800 & -2.20989100 & 0.54219800 \\
\hline $\mathrm{C}$ & 1.61959000 & -3.48202900 & 0.35938800 \\
\hline $\mathrm{C}$ & 0.32428500 & -3.66742800 & -0.12938400 \\
\hline $\mathrm{C}$ & -0.49987900 & -2.58479300 & -0.48700900 \\
\hline $\mathrm{H}$ & 3.19504100 & -2.06267300 & 0.91414600 \\
\hline $\mathrm{H}$ & 2.21179200 & -4.35728700 & 0.60926000 \\
\hline $\mathrm{H}$ & -0.05187500 & -4.67908600 & -0.24413300 \\
\hline $\mathrm{H}$ & -1.49972100 & -2.72361000 & -0.88350400 \\
\hline $\mathrm{N}$ & 1.27694000 & 0.23928500 & 0.16100400 \\
\hline $\mathrm{C}$ & 2.29659900 & 1.13276100 & -0.01061100 \\
\hline $\mathrm{O}$ & 3.46276500 & 0.89655200 & 0.20505100 \\
\hline $\mathrm{O}$ & 1.80725200 & 2.32555400 & -0.40699600 \\
\hline $\mathrm{C}$ & -0.07957000 & 0.17219300 & -0.45913400 \\
\hline $\mathrm{H}$ & -0.06717600 & 0.50893300 & -1.50127500 \\
\hline $\mathrm{C}$ & -1.17433900 & 0.84454700 & 0.31843600 \\
\hline $\mathrm{C}$ & -0.97384600 & 1.68581800 & 1.32990900 \\
\hline $\mathrm{H}$ & 0.03250000 & 1.94288200 & 1.64217300 \\
\hline $\mathrm{C}$ & 2.80050400 & 3.33662100 & -0.57064400 \\
\hline $\mathrm{H}$ & 2.26270800 & 4.22642400 & -0.89448000 \\
\hline $\mathrm{H}$ & 3.31586300 & 3.52046200 & 0.37455500 \\
\hline $\mathrm{H}$ & 3.53339800 & 3.03399100 & -1.32156000 \\
\hline $\mathrm{C}$ & -2.53340200 & 0.45892500 & -0.15223600 \\
\hline $\mathrm{O}$ & -2.71843400 & -0.33394800 & -1.04918400 \\
\hline $\mathrm{O}$ & -3.52496600 & 1.07002500 & 0.50802200 \\
\hline $\mathrm{C}$ & -4.83925800 & 0.70620300 & 0.08681100 \\
\hline $\mathrm{H}$ & -5.00416600 & -0.36265700 & 0.23964700 \\
\hline $\mathrm{H}$ & -5.51878100 & 1.29237900 & 0.70306700 \\
\hline $\mathrm{H}$ & -4.97786200 & 0.93895700 & -0.97111900 \\
\hline $\mathrm{H}$ & -1.81005200 & 2.13100800 & 1.85720100 \\
\hline
\end{tabular}




\section{X-ray Crystallographic Analysis}

\section{$\mathrm{Cs}_{2} \mathrm{CO}_{3}$ catalyzed self-cyclization product $-2 \mathrm{t}$}

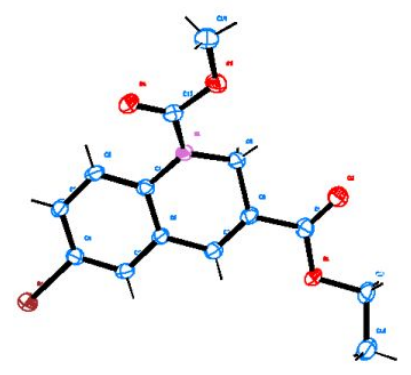

ORTEP diagram of $\mathbf{2 t}$. Thermal ellipsoids are shown at the 50\% probability level. A colorless stick-shaped crystal of $\mathbf{2 t}$ for X-ray diffraction was obtained by slowly volatilizing a saturated solution of $\mathbf{2} \mathbf{t}$ in hexane/chloroform (5:1). The X-ray intensity data was measured on a Rigaku 007 Saturn 70 single crystal diffractometer.

\begin{tabular}{|c|c|}
\hline CCDC number & 2023956 \\
\hline Identification code & $2 t$ \\
\hline Empirical formula & $\mathrm{C}_{14} \mathrm{H}_{14} \mathrm{BrNO}_{4}$ \\
\hline Formula weight & 340.17 \\
\hline Temperature/K & $113(2)$ \\
\hline Crystal system & monoclinic \\
\hline Space group & $\mathrm{P} 2_{1} / \mathrm{c}$ \\
\hline $\mathrm{a} / \AA$ & $4.0104(8)$ \\
\hline $\mathrm{b} / \AA \AA$ & $27.174(5)$ \\
\hline $\mathrm{c} / \AA \AA$ & $12.516(3)$ \\
\hline$\alpha /^{\circ}$ & 90 \\
\hline$\beta /{ }^{\circ}$ & $95.14(3)$ \\
\hline$\gamma /{ }^{\circ}$ & 90 \\
\hline Volume $/ \AA^{3}$ & $1358.5(5)$ \\
\hline $\mathrm{Z}$ & 4 \\
\hline$\rho_{\text {calc }} \mathrm{g} / \mathrm{cm}^{3}$ & 1.663 \\
\hline$\mu / \mathrm{mm}^{-1}$ & 3.039 \\
\hline $\mathrm{F}(000)$ & 688.0 \\
\hline Crystal size $/ \mathrm{mm}^{3}$ & $0.200 \times 0.180 \times 0.120$ \\
\hline Radiation & $\operatorname{MoK} \alpha(\lambda=0.71073)$ \\
\hline $2 \Theta$ range for data collection ${ }^{\circ}$ & 4.434 to 55.736 \\
\hline Index ranges & $-5 \leq \mathrm{h} \leq 5,-35 \leq \mathrm{k} \leq 35,-16 \leq 1 \leq 16$ \\
\hline Reflections collected & 16083 \\
\hline Independent reflections & $3228\left[\mathrm{R}_{\text {int }}=0.0600, \mathrm{R}_{\text {sigma }}=0.0483\right]$ \\
\hline Data/restraints/parameters & $3228 / 0 / 184$ \\
\hline Goodness-of-fit on $\mathrm{F}^{2}$ & 1.067 \\
\hline Final $R$ indexes $[I>=2 \sigma(I)]$ & $\mathrm{R}_{1}=0.0421, \mathrm{wR}_{2}=0.1209$ \\
\hline Final $\mathrm{R}$ indexes [all data] & $\mathrm{R}_{1}=0.0548, \mathrm{wR}_{2}=0.1510$ \\
\hline Largest diff. peak/hole / e $\AA^{-3}$ & $0.72 /-0.80$ \\
\hline
\end{tabular}

Derivatization of quinuclidine catalyzed self-cyclization product $-4 \mathrm{~b}$ 


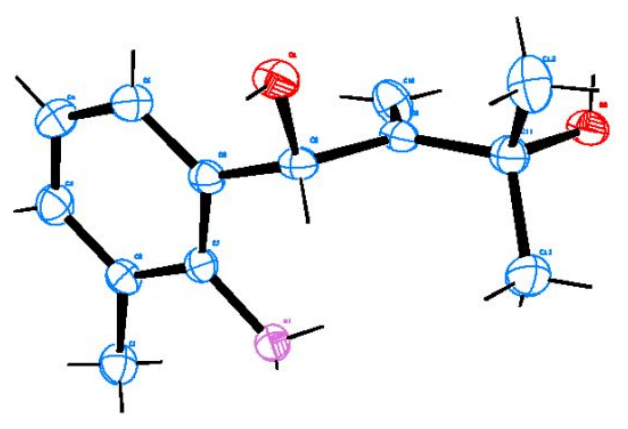

CCDC number

Identification code

Empirical formula

Formula weight

Temperature/K

Crystal system

Space group

$\mathrm{a} / \AA$

$\mathrm{b} / \AA$

$\mathrm{c} / \AA ̊$

$\alpha /{ }^{\circ}$

$\beta /{ }^{\circ}$

$\gamma /{ }^{\circ}$

Volume $/ \AA^{3}$

Z

$\rho_{\text {calc }} \mathrm{g} / \mathrm{cm}^{3}$

$\mu / \mathrm{mm}^{-1}$

$\mathrm{F}(000)$

Crystal size $/ \mathrm{mm}^{3}$

Radiation

$2 \Theta$ range for data collection/ ${ }^{\circ}$

Index ranges

Reflections collected

Independent reflections

Data/restraints/parameters

Goodness-of-fit on $\mathrm{F}^{2}$

Final $\mathrm{R}$ indexes $[\mathrm{I}>=2 \sigma(\mathrm{I})]$

Final $\mathrm{R}$ indexes [all data]

Largest diff. peak/hole / e $\AA^{-3}$
ORTEP diagram of $\mathbf{4 b}$. Thermal ellipsoids are shown at the $50 \%$ probability level. A colorless stick-shaped crystal of $\mathbf{4 b}$ for X-ray diffraction was obtained by slowly volatilizing a saturated solution of 4b in hexane/ethyl acetate (4:1). The X-ray intensity data was measured on a Rigaku 007 Saturn 70 single crystal diffractometer.
2023953

$4 b$

$\mathrm{C}_{13} \mathrm{H}_{19} \mathrm{NO}_{2}$

221.29

113(2)

monoclinic

$\mathrm{P} 21 / \mathrm{n}$

$10.975(2)$

8.6524(17)

13.244(3)

90

97.02(3)

90

1248.1(4)

4

1.178

0.079

480.0

$0.200 \times 0.180 \times 0.120$

$\operatorname{MoK} \alpha(\lambda=0.71073)$

4.556 to 55.752

$-14 \leq \mathrm{h} \leq 13,-11 \leq \mathrm{k} \leq 11,-17 \leq 1 \leq 15$

14653

$2981\left[\mathrm{R}_{\text {int }}=0.0545, \mathrm{R}_{\text {sigma }}=0.0422\right]$

$2981 / 3 / 159$

1.074

$\mathrm{R}_{1}=0.0579, \mathrm{wR}_{2}=0.1450$

$\mathrm{R}_{1}=0.0798, \mathrm{wR}_{2}=0.1664$

$0.44 /-0.32$

Derivatization of quinuclidine catalyzed self-cyclization product $-4 d$ 


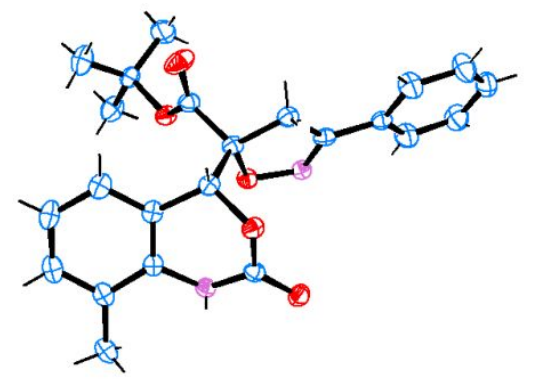

ORTEP diagram of $\mathbf{4 d}$. Thermal ellipsoids are shown at the $50 \%$ probability level. A colorless stick-shaped crystal of $\mathbf{4 d}$ for X-ray diffraction was obtained by slowly volatilizing a saturated solution of $\mathbf{4 d}$ in hexane/chloroform (4:1). The X-ray intensity data was measured on a Rigaku 007 Saturn 70 single crystal diffractometer.

CCDC number

Identification code

Empirical formula

Formula weight

Temperature/K

Crystal system

Space group

$\mathrm{a} / \AA$

$\mathrm{b} / \AA$

$\mathrm{c} / \AA ̊$

$\alpha /{ }^{\circ}$

$\beta /{ }^{\circ}$

$\gamma /{ }^{\circ}$

Volume $/ \AA^{3}$

Z

$\rho_{\text {calc }} \mathrm{g} / \mathrm{cm}^{3}$

$\mu / \mathrm{mm}^{-1}$

$\mathrm{F}(000)$

Crystal size $/ \mathrm{mm}^{3}$

Radiation

$2 \Theta$ range for data collection/ ${ }^{\circ}$

Index ranges

Reflections collected

Independent reflections

Data/restraints/parameters

Goodness-of-fit on $\mathrm{F}^{2}$

Final $\mathrm{R}$ indexes $[\mathrm{I}>=2 \sigma(\mathrm{I})]$

Final R indexes [all data]

Largest diff. peak/hole / e $\AA^{-3}$
2023955

4d

$\mathrm{C}_{23} \mathrm{H}_{24} \mathrm{~N}_{2} \mathrm{O}_{5}$

408.44

113.15

monoclinic

$\mathrm{P} 2 / \mathrm{n}$

14.4670(12)

10.8524(6)

$14.7802(11)$

90

$117.483(10)$

90

2058.6(3)

4

1.318

0.093

864.0

$0.2 \times 0.18 \times 0.16$

$\operatorname{MoK} \alpha(\lambda=0.71073)$

4.872 to 52.74

$-18 \leq \mathrm{h} \leq 17,-13 \leq \mathrm{k} \leq 13,-18 \leq 1 \leq 18$

17640

$4205\left[\mathrm{R}_{\text {int }}=0.0624, \mathrm{R}_{\text {sigma }}=0.0488\right]$

$4205 / 0 / 275$

1.069

$\mathrm{R}_{1}=0.0507, \mathrm{wR}_{2}=0.1127$

$\mathrm{R}_{1}=0.0702, \mathrm{wR}_{2}=0.1232$

$0.27 /-0.29$ 


\section{NMR Spectra}

4.1. o-amino-acylation aryl MBH carbonates 1b'-1f'
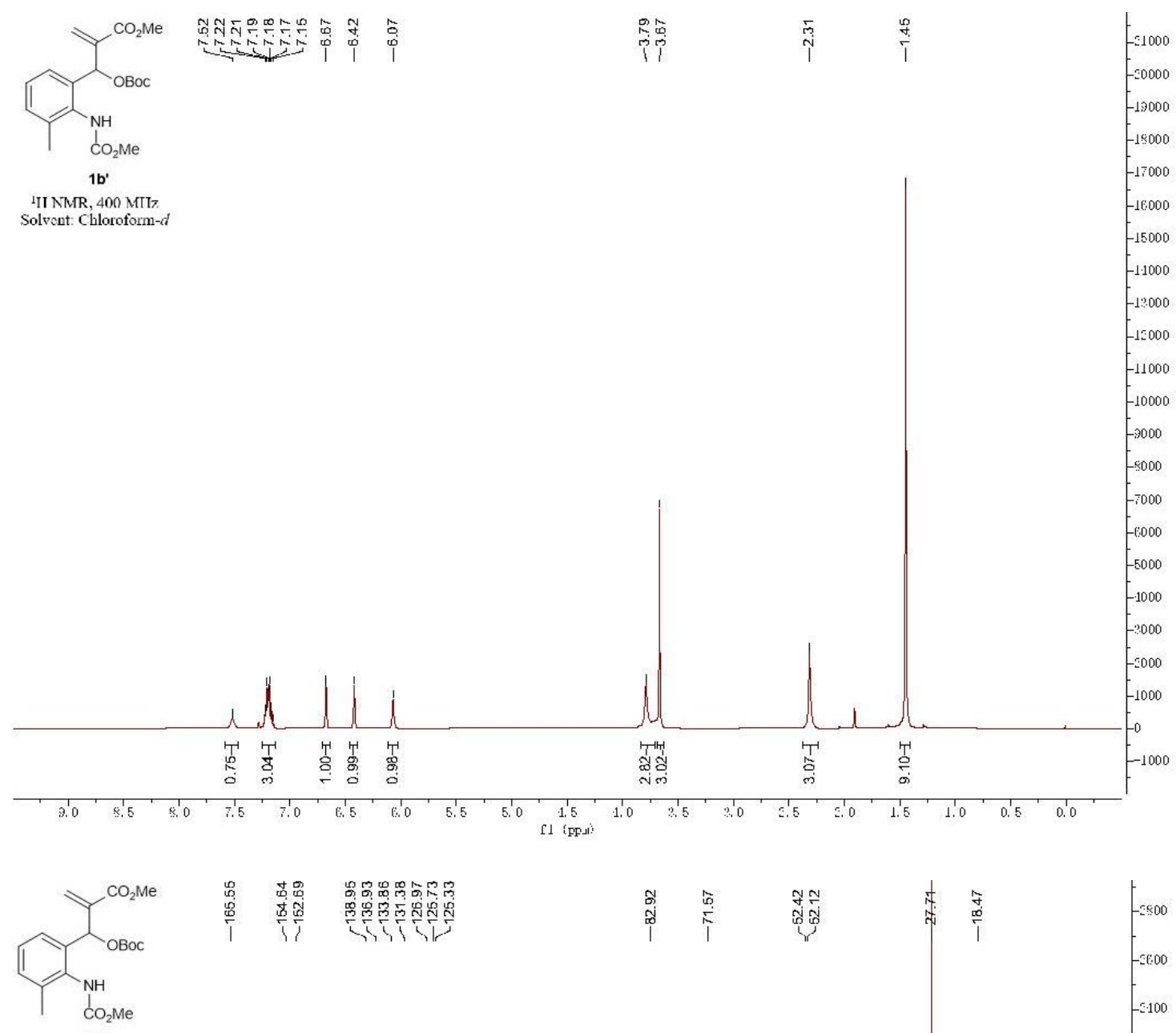

1b'

$\left.{ }^{19} \mathrm{C}:{ }^{1} \mathrm{H}\right\} \mathrm{NMR}, 101 \mathrm{MH}$.
Solvent: Chloroform- $d$

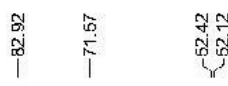

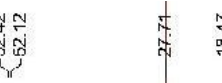

$-\leqslant 00$

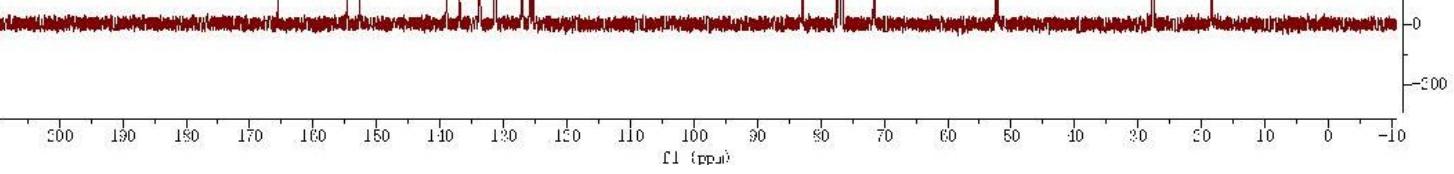




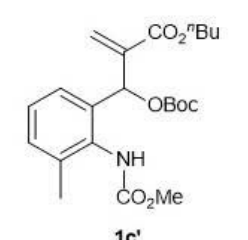

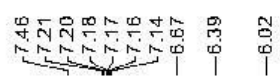

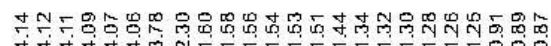

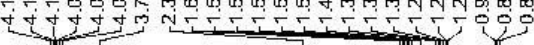

$-12000$

Solvent: Chloroform- $d$

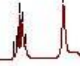

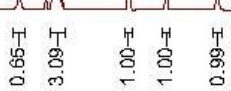

岁管

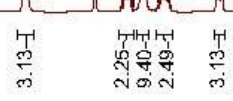

कृष ले

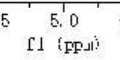

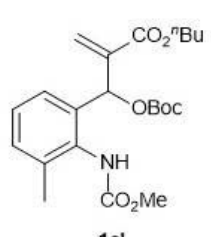

\section{点}

TMR, $101 \mathrm{MH}:$

Solvent: Chloroform-d

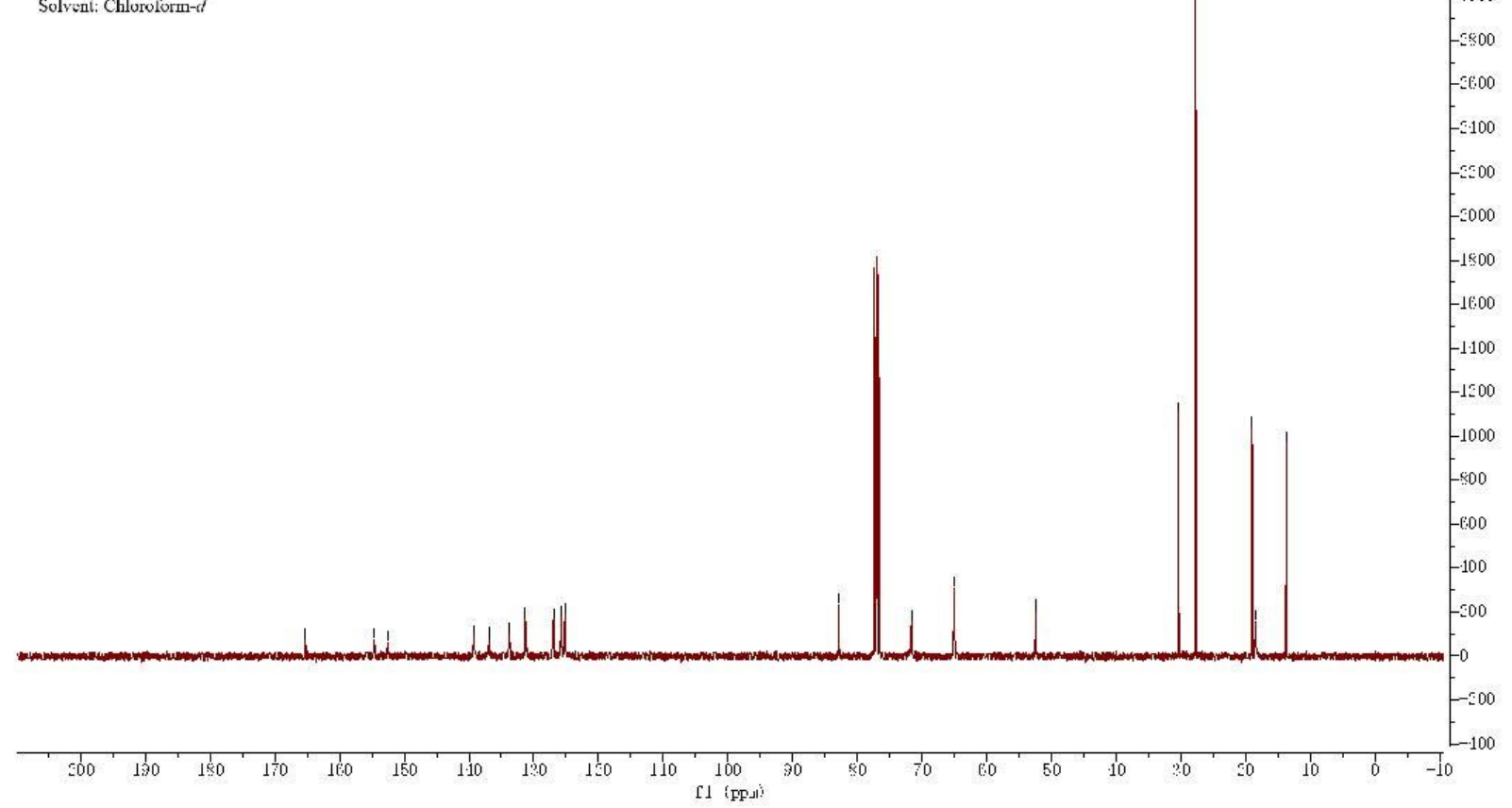



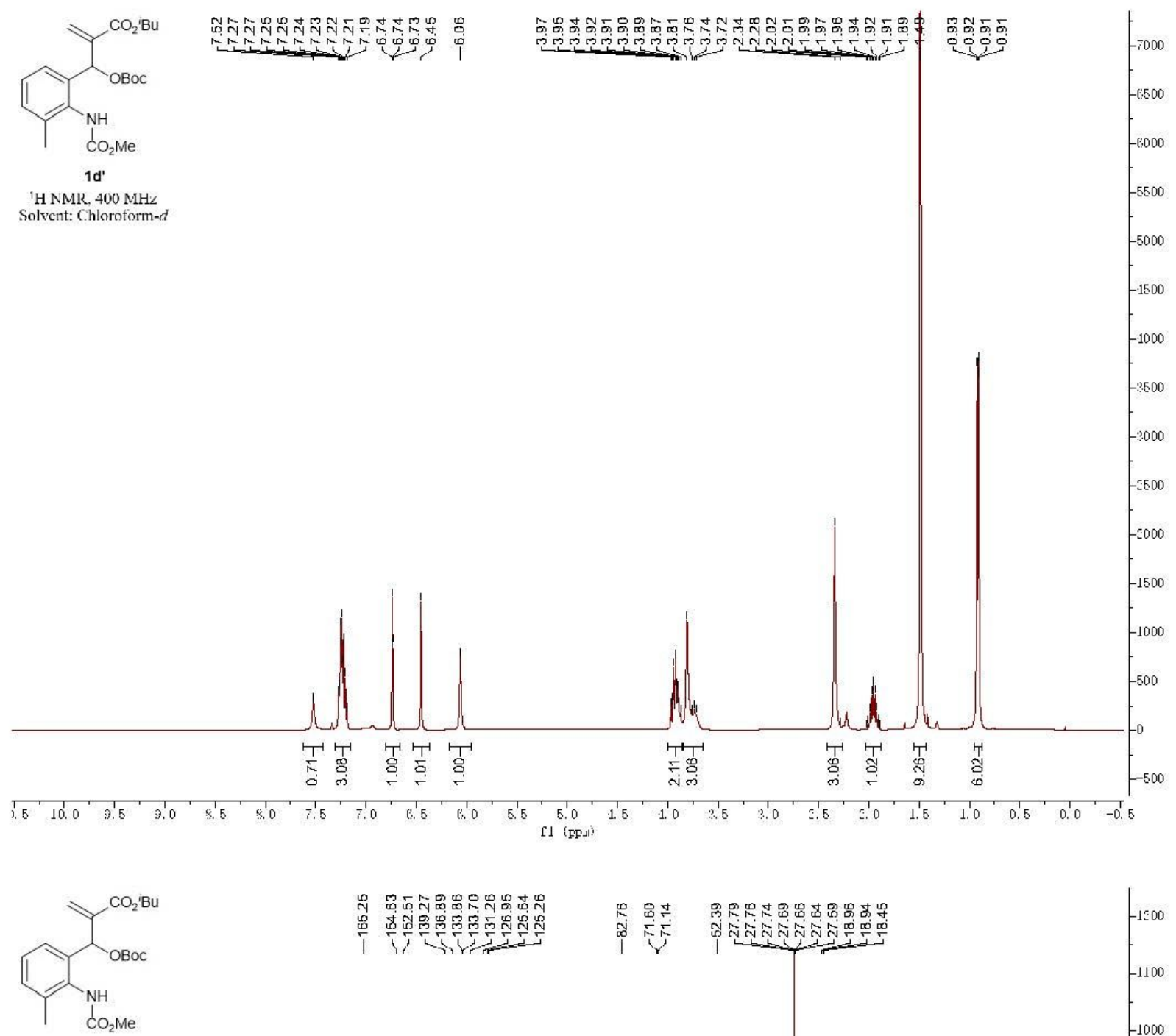

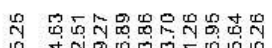
। 告

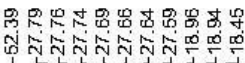

$1 d^{\prime}$

1sC'11H NMR, 101 MIK Solvent: Chloroform-d

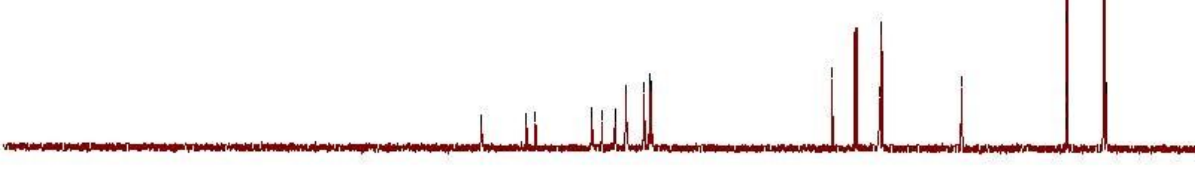



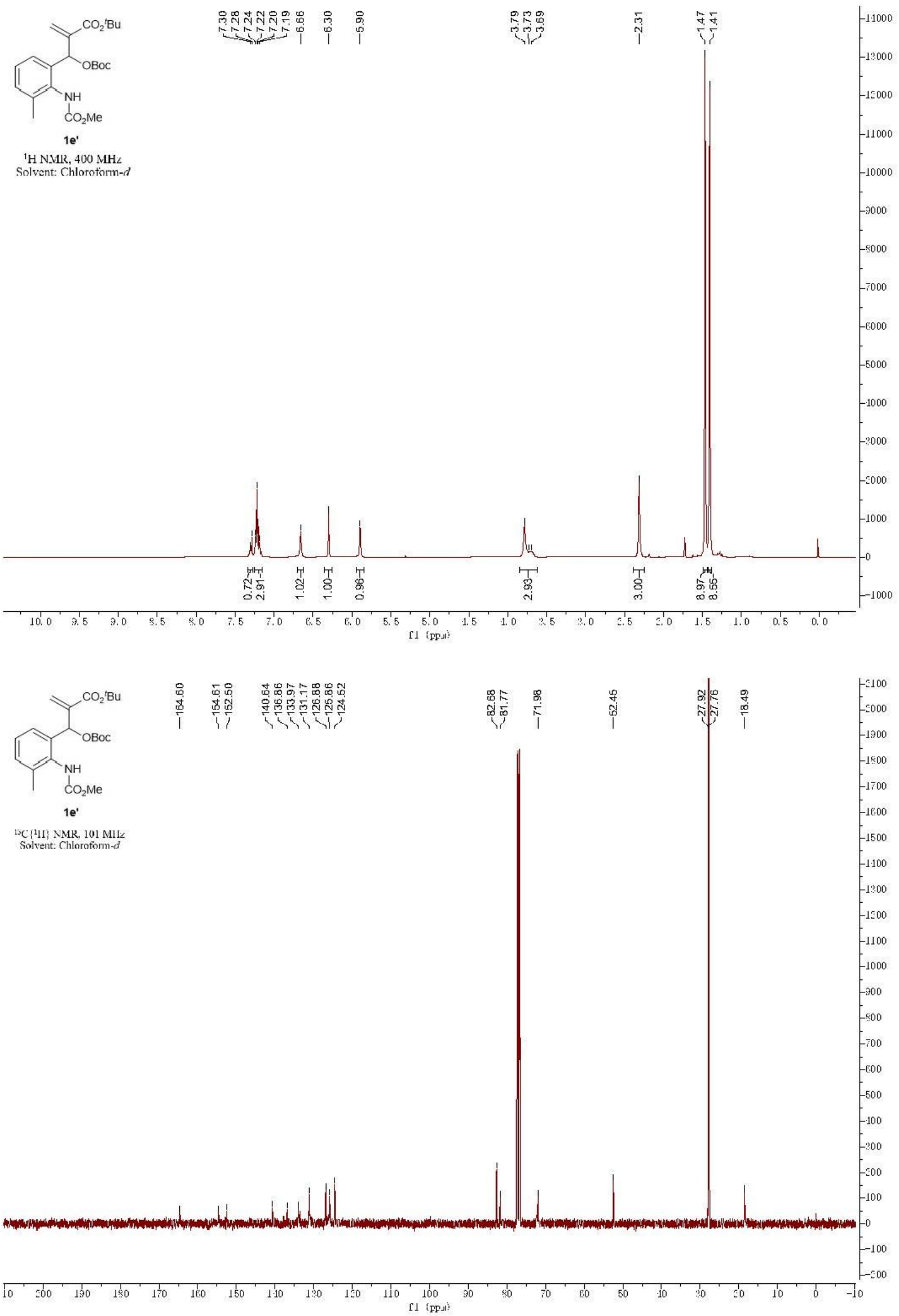

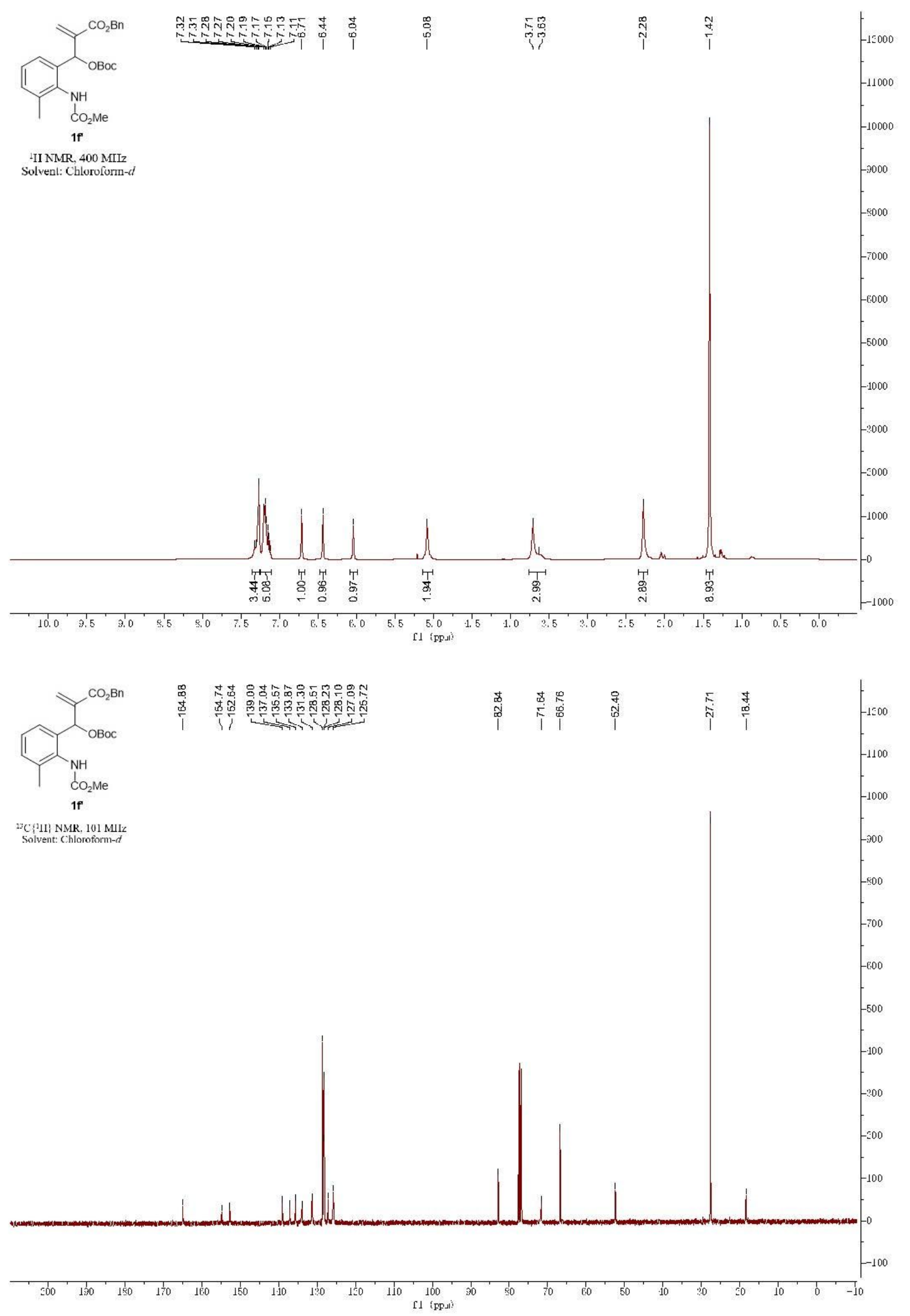
4.2. $\mathrm{Cs}_{2} \mathrm{CO}_{3}$ catalyzed intramolecular cyclization products 2

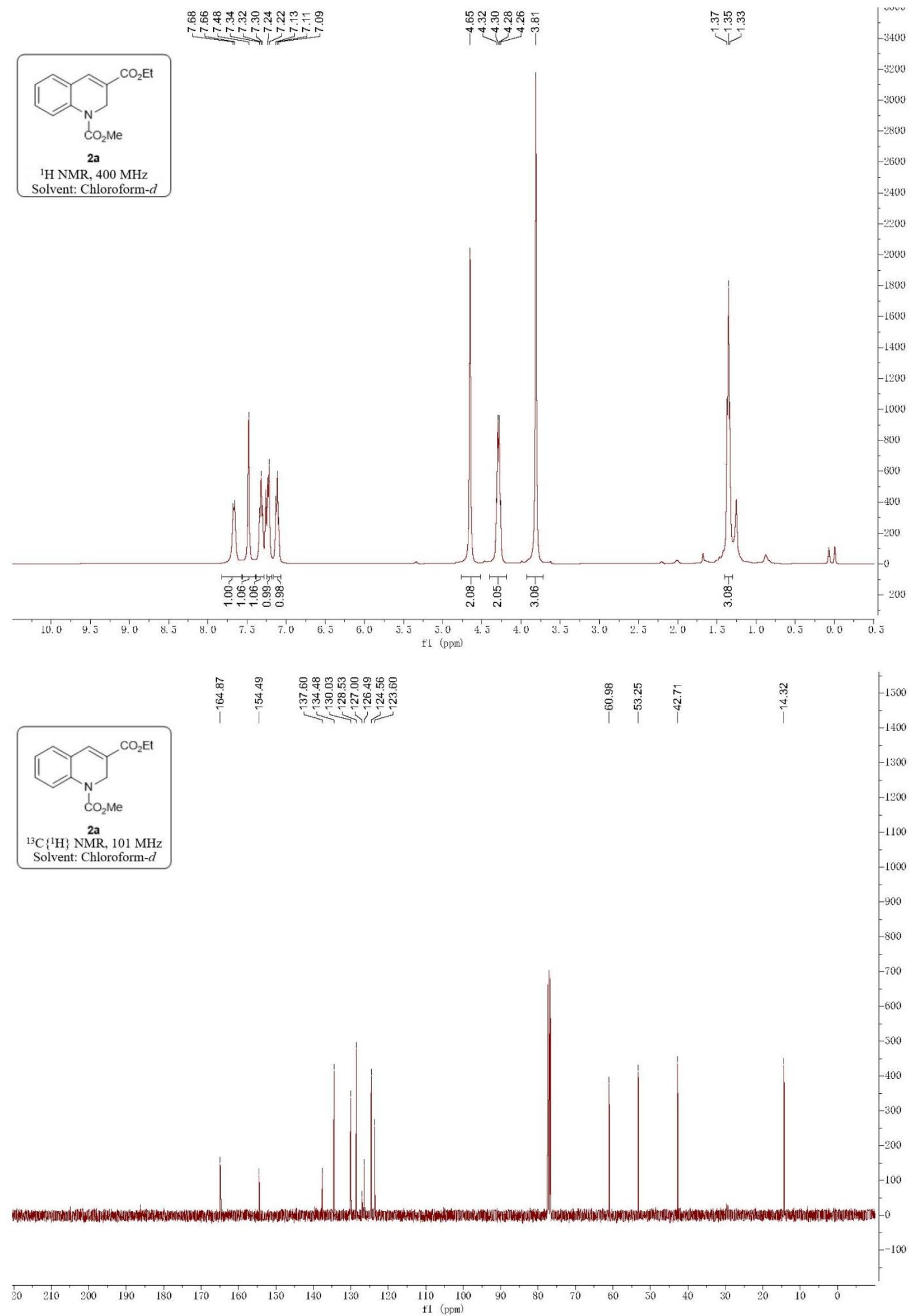




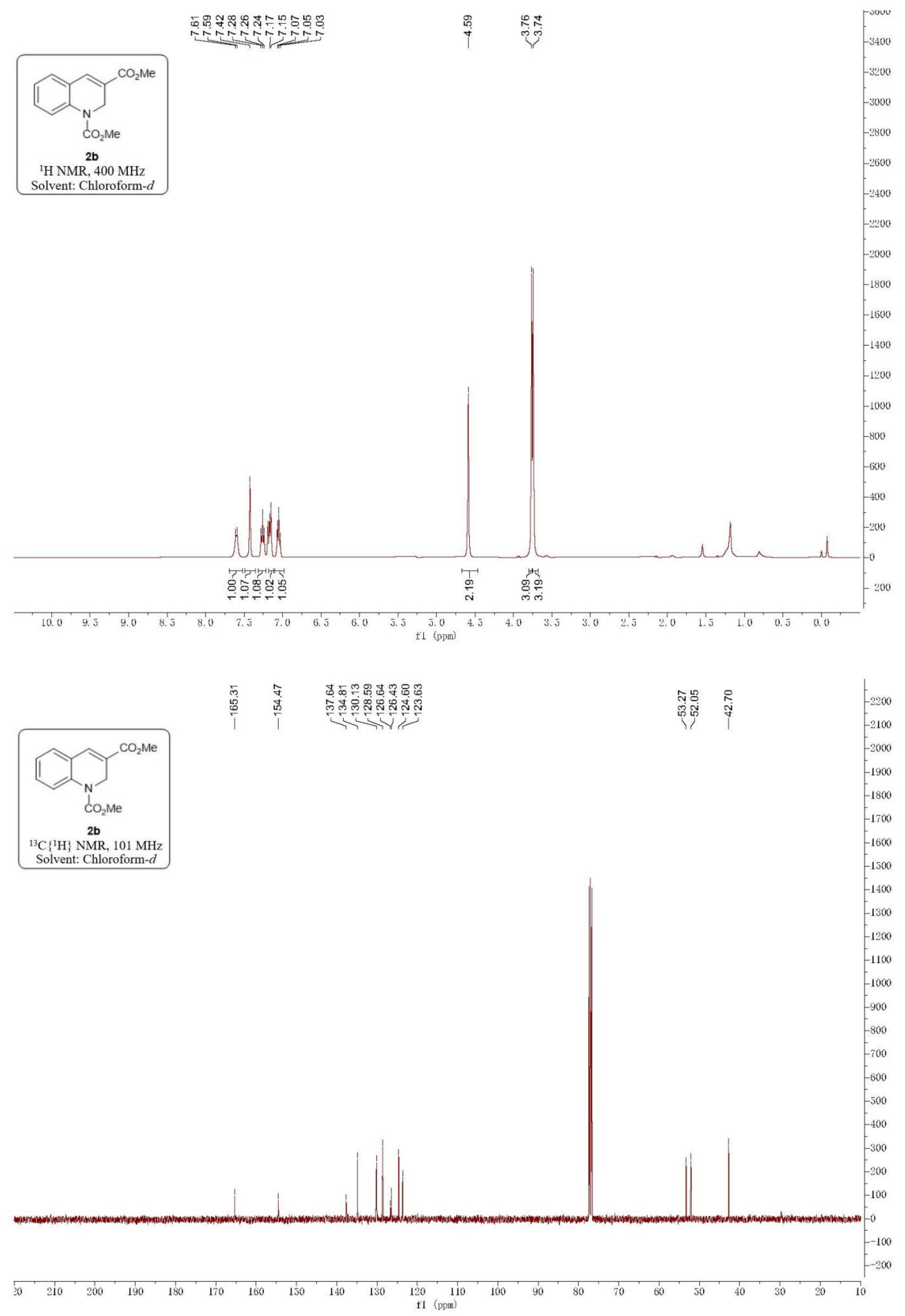



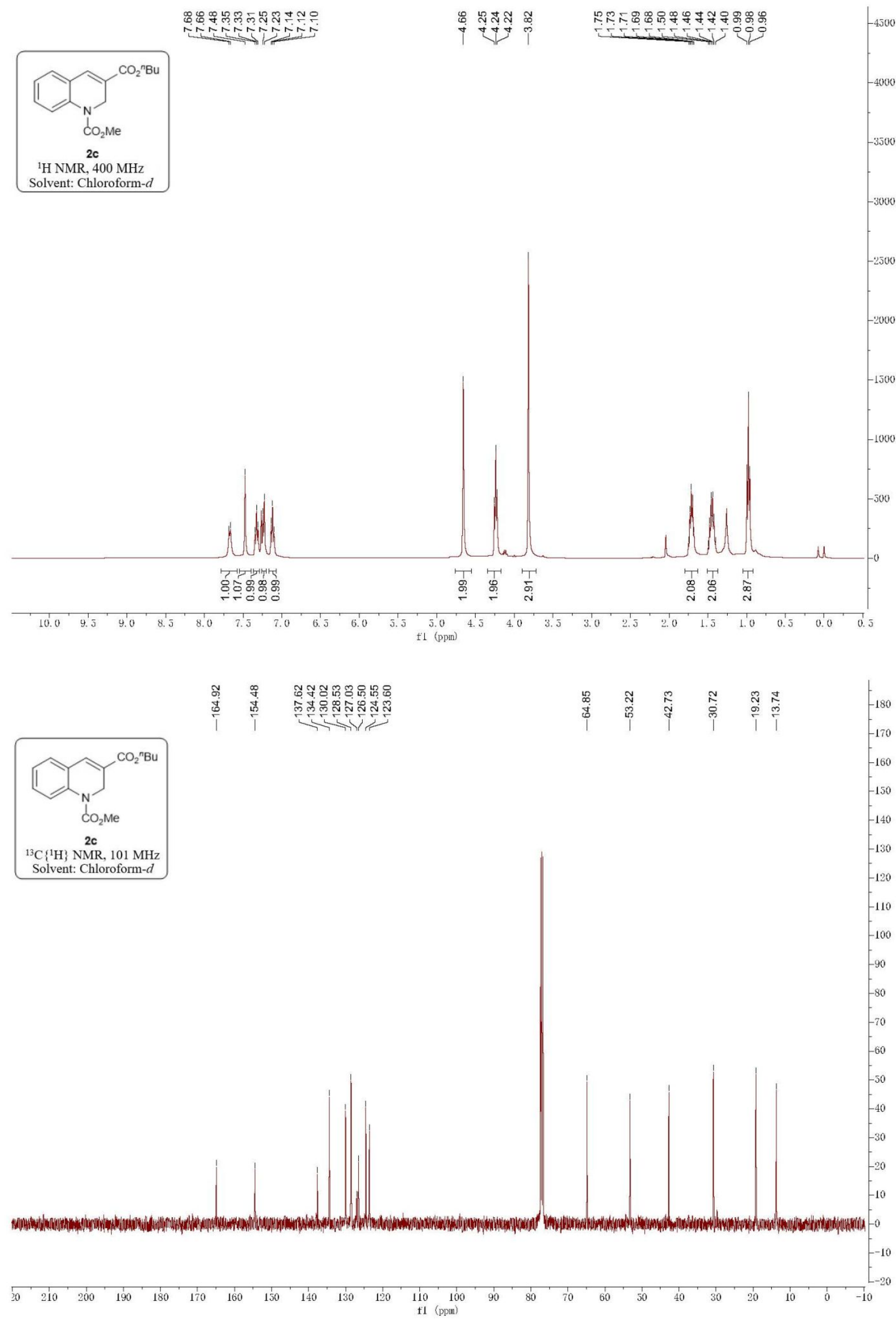


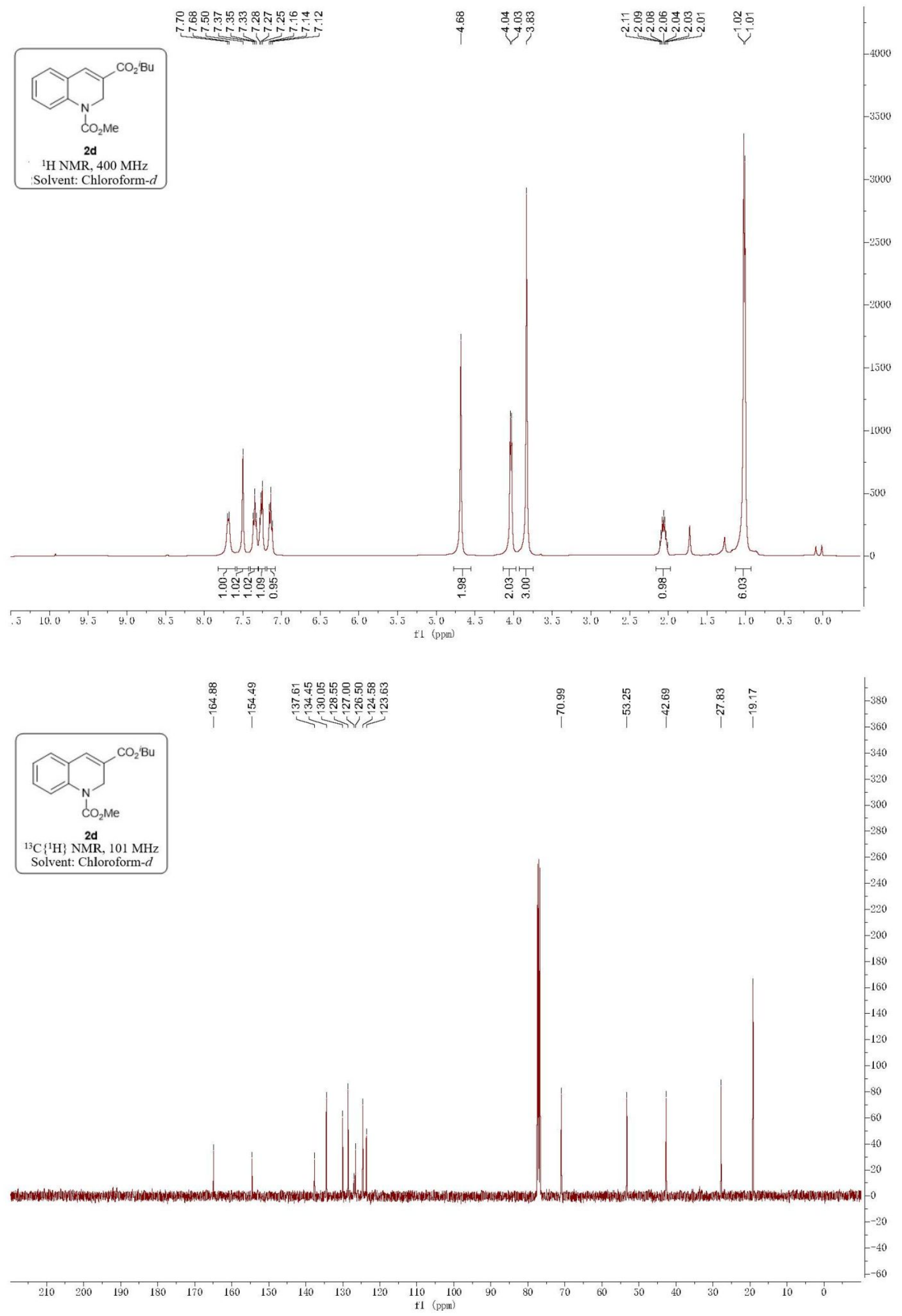



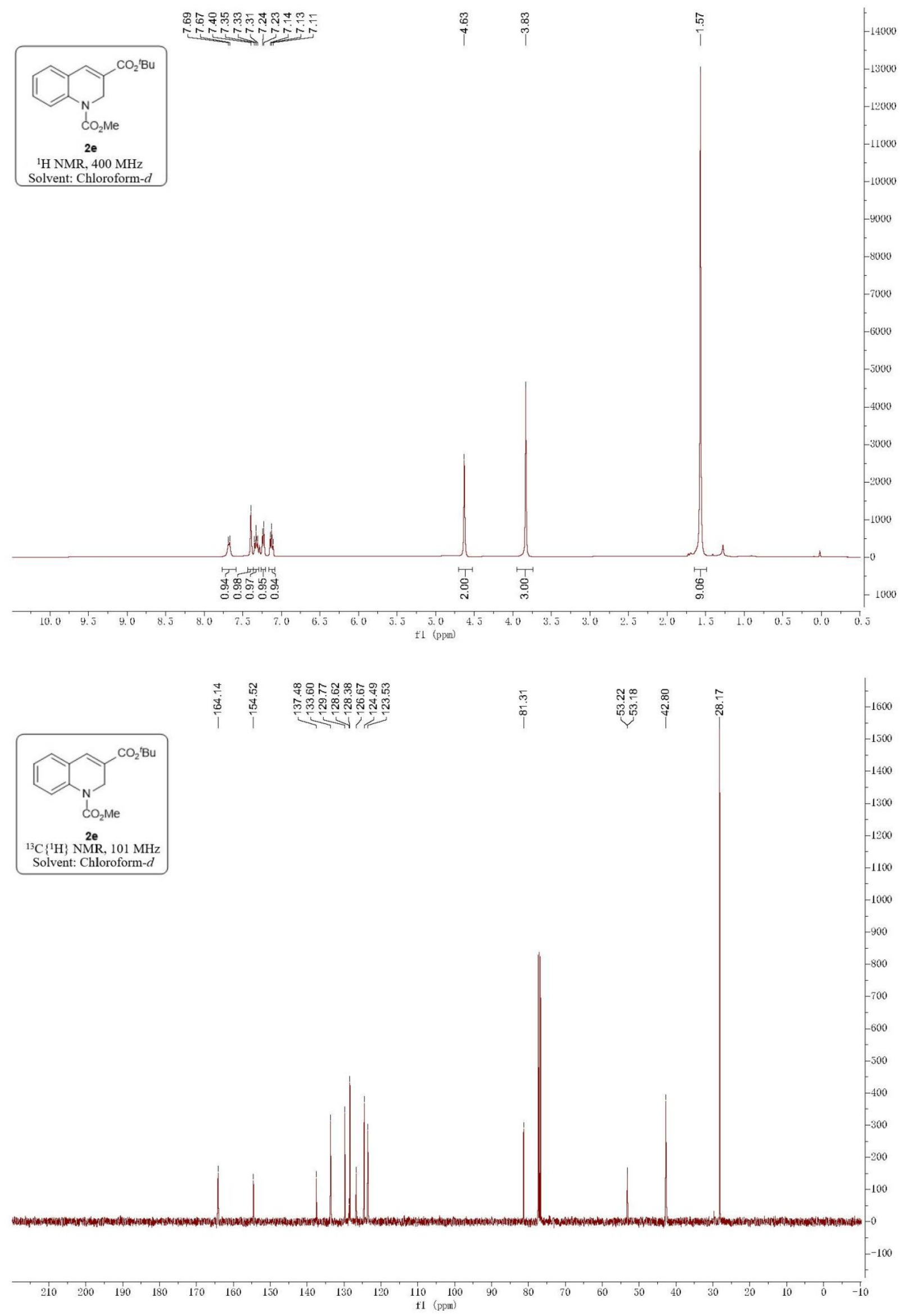

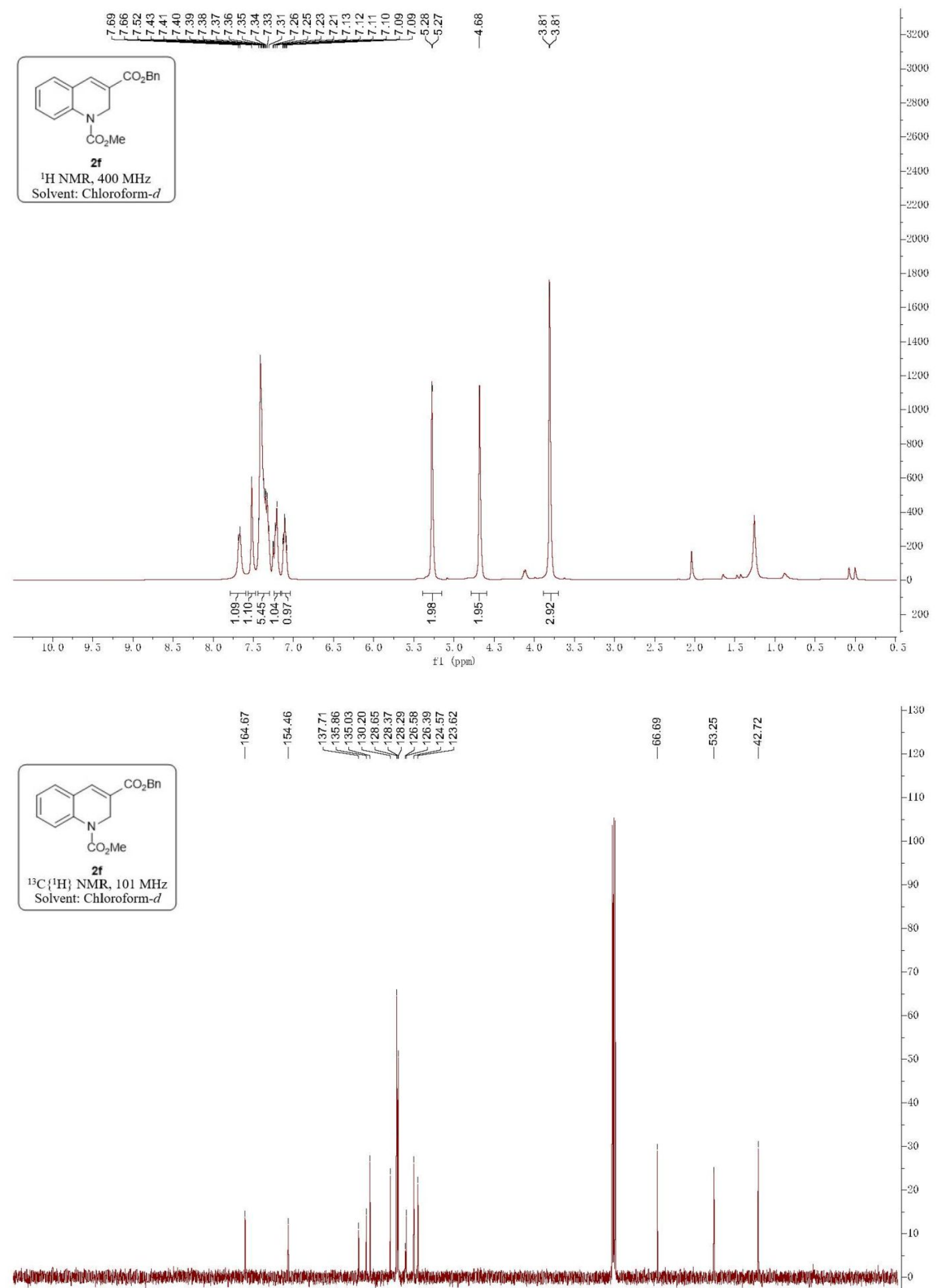

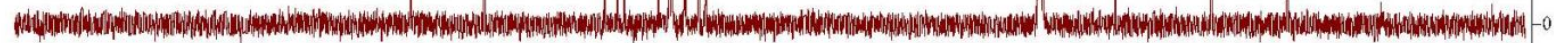

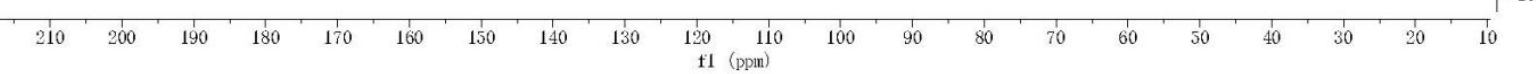



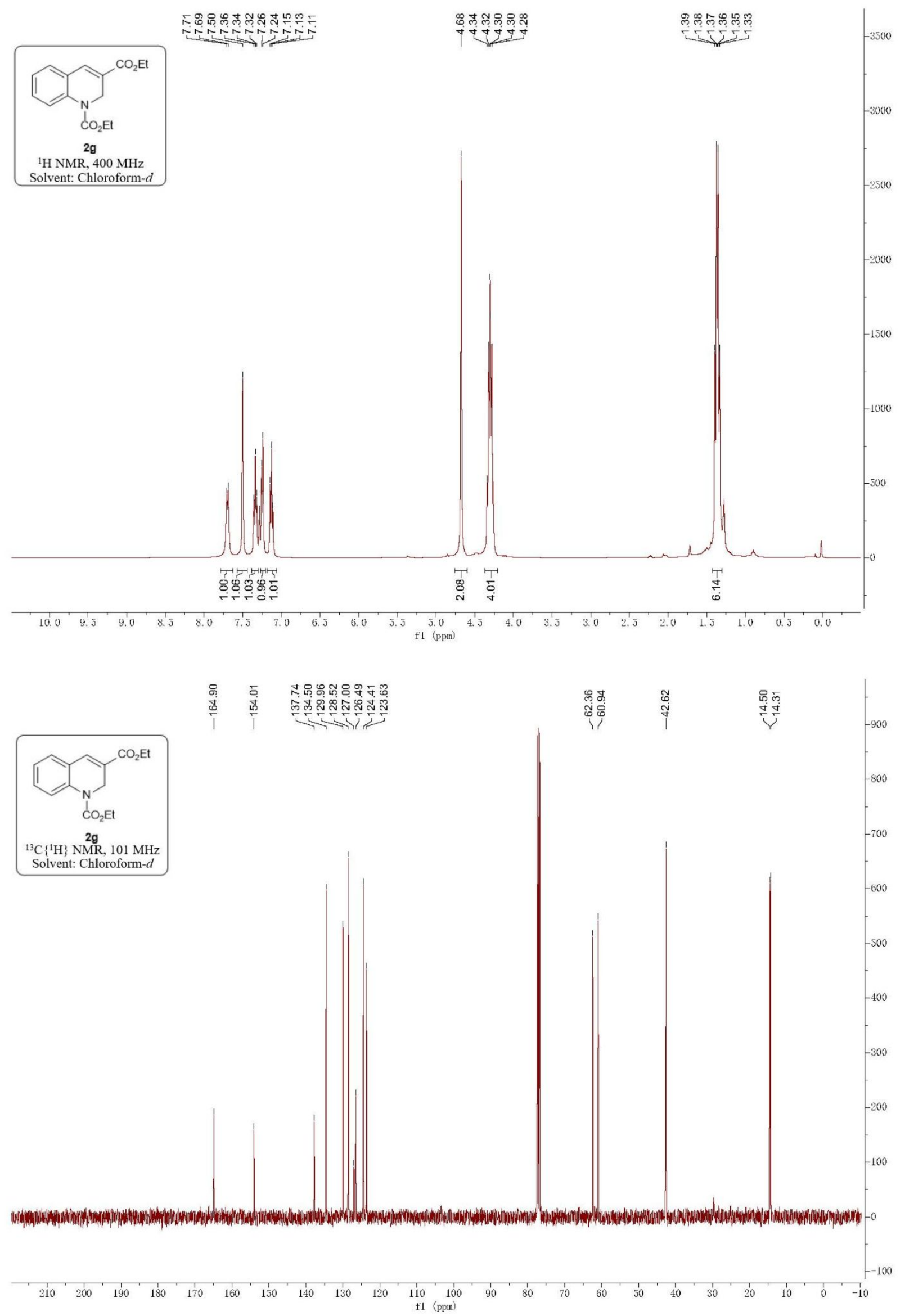

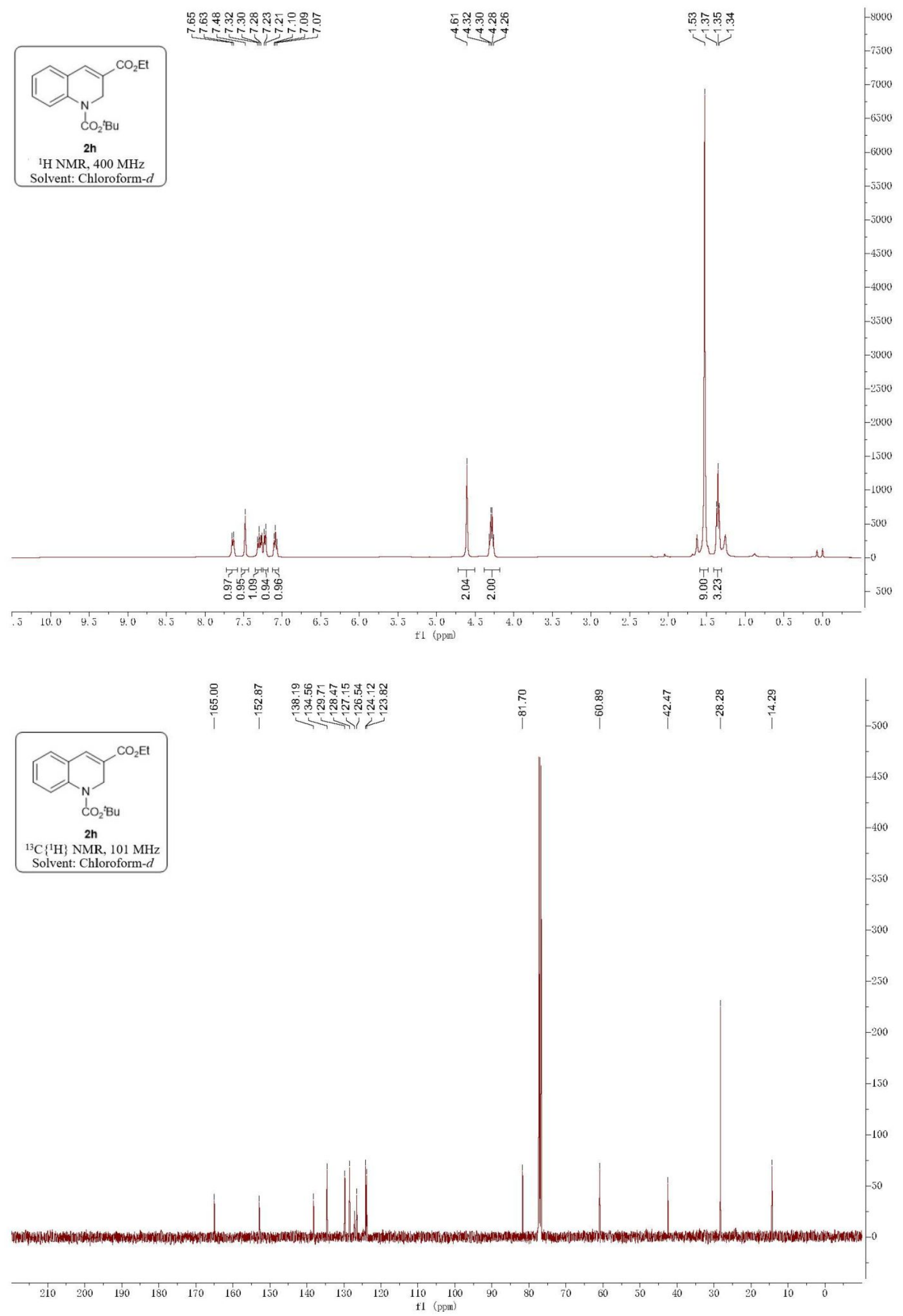

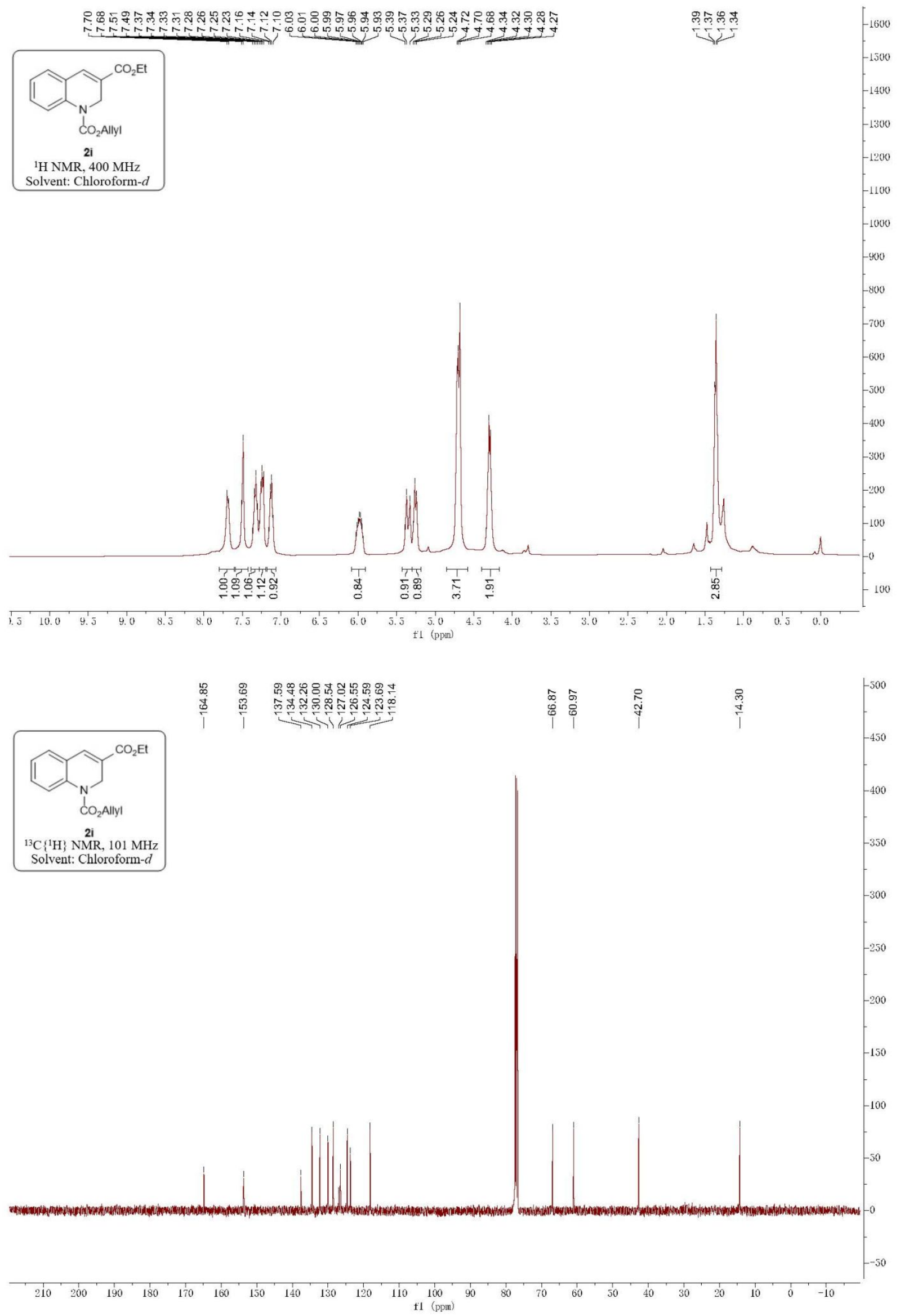

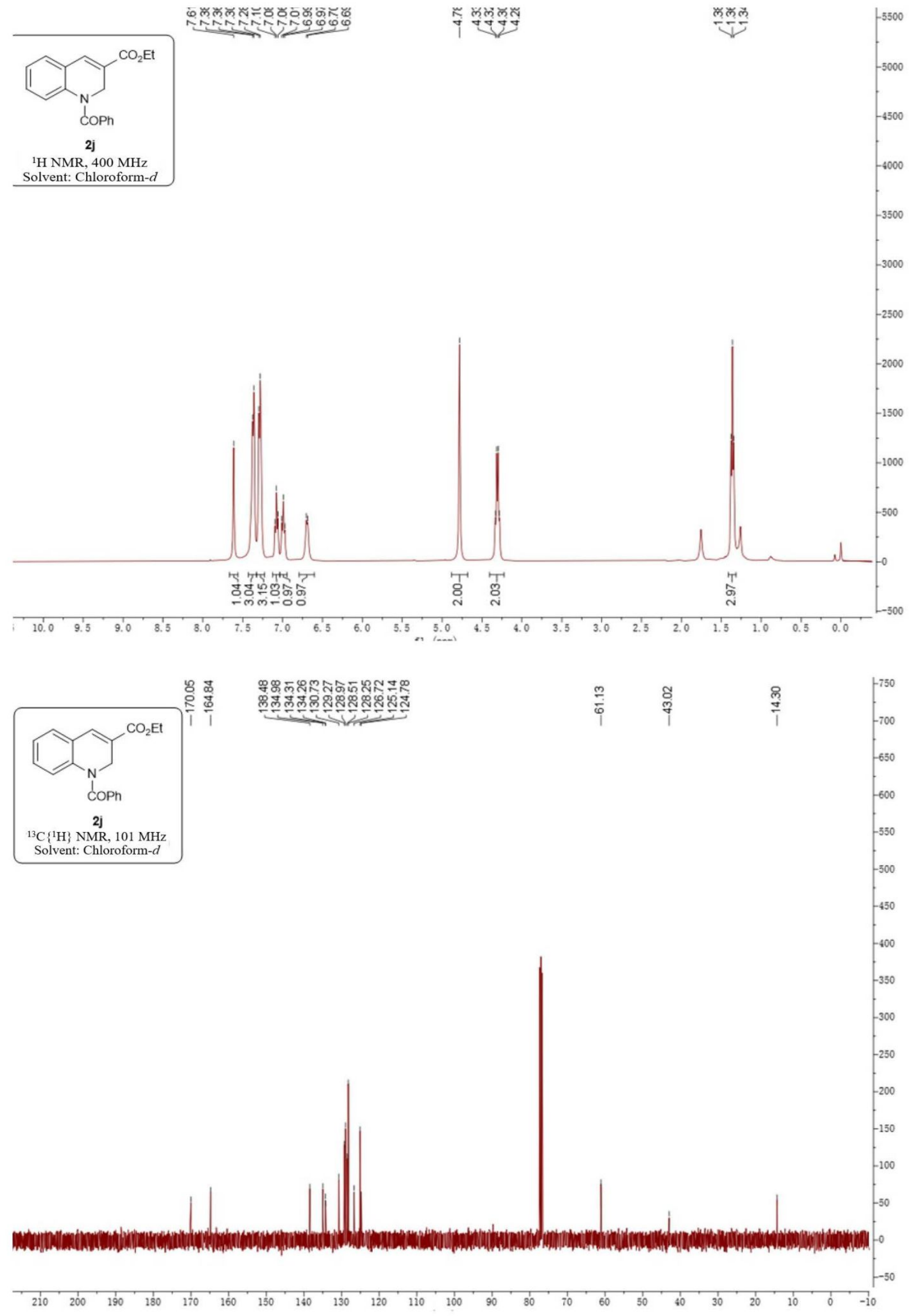

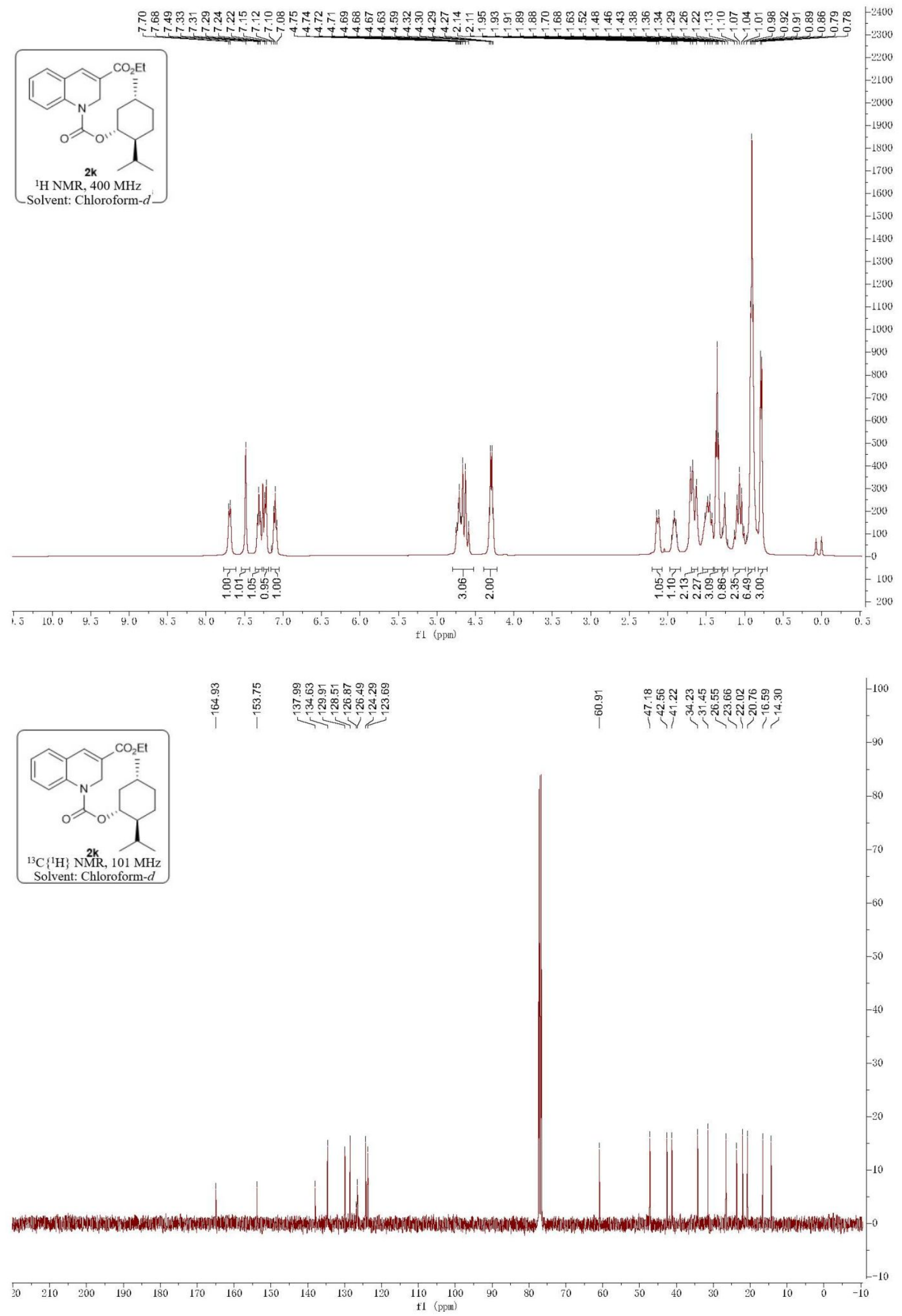

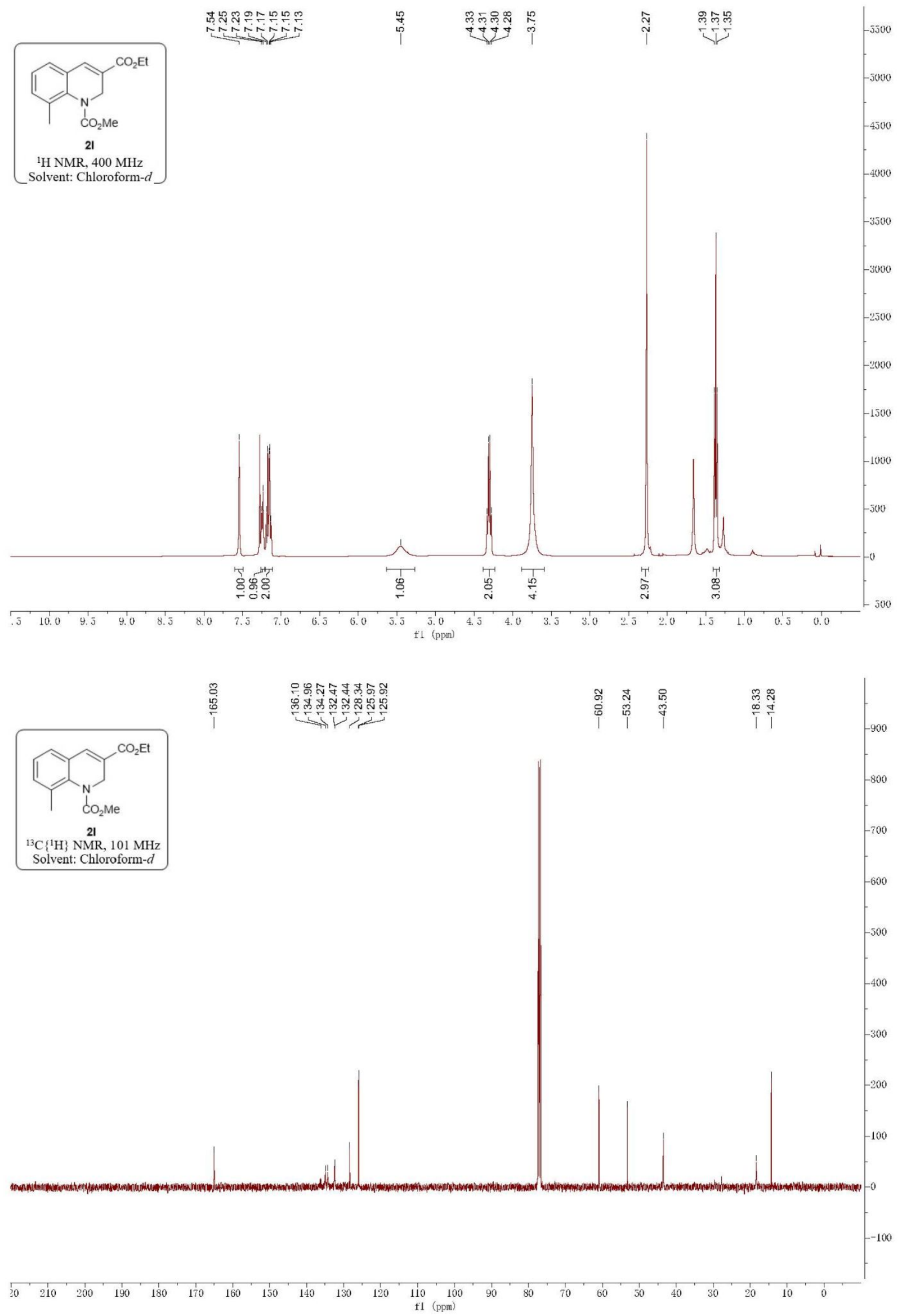

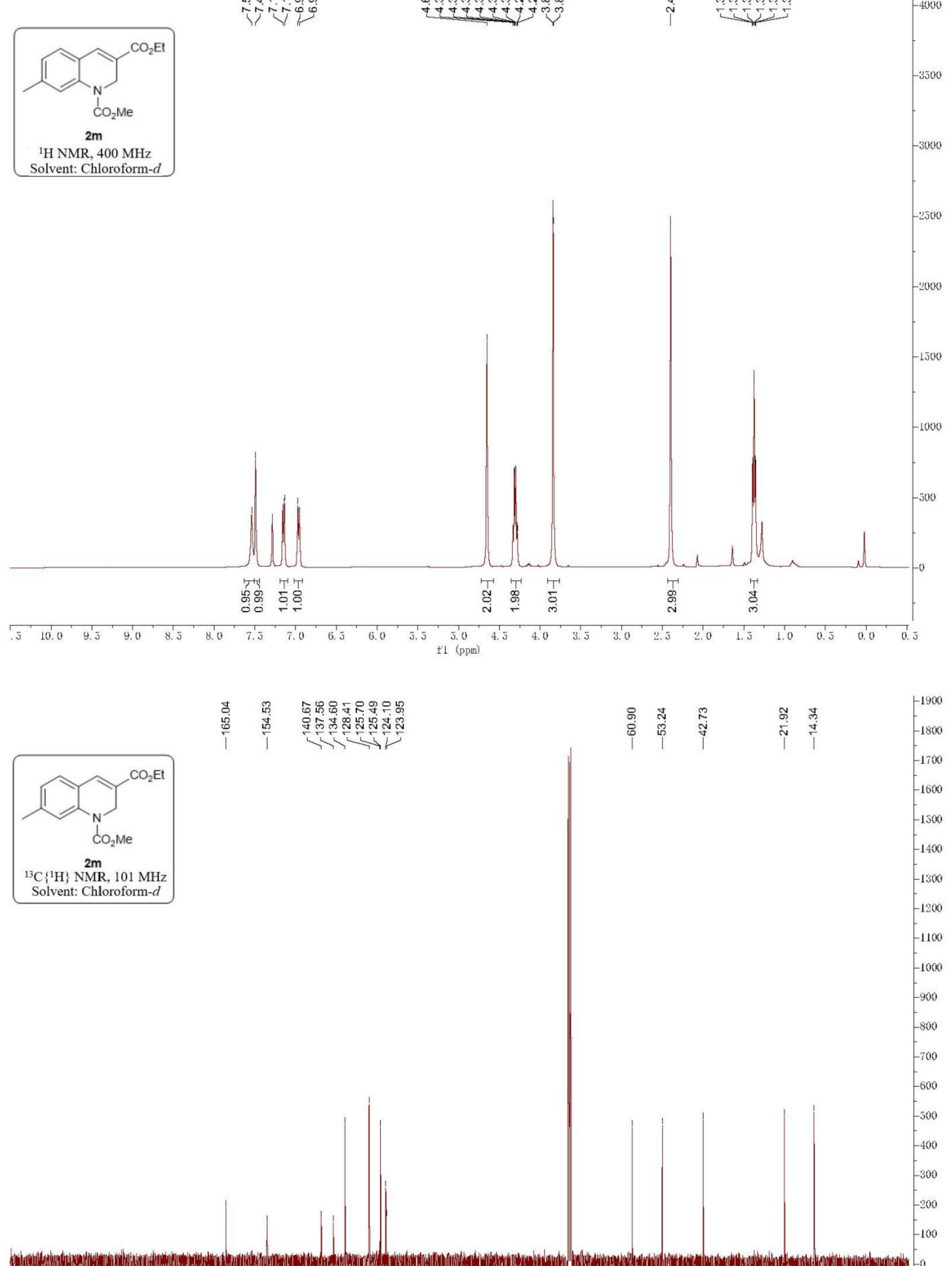

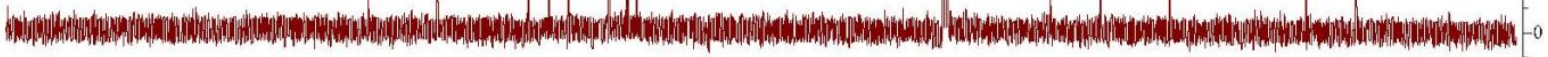

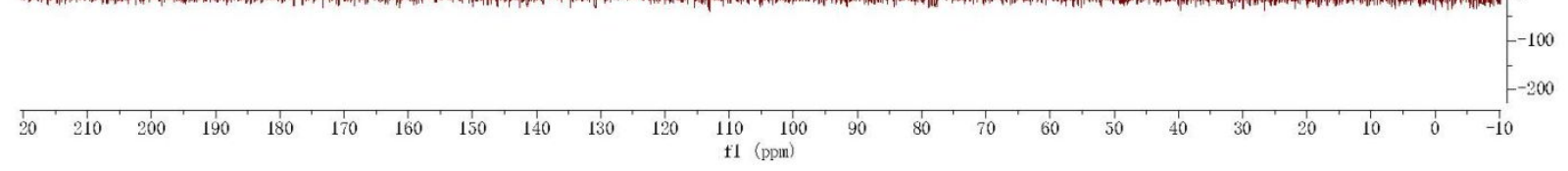



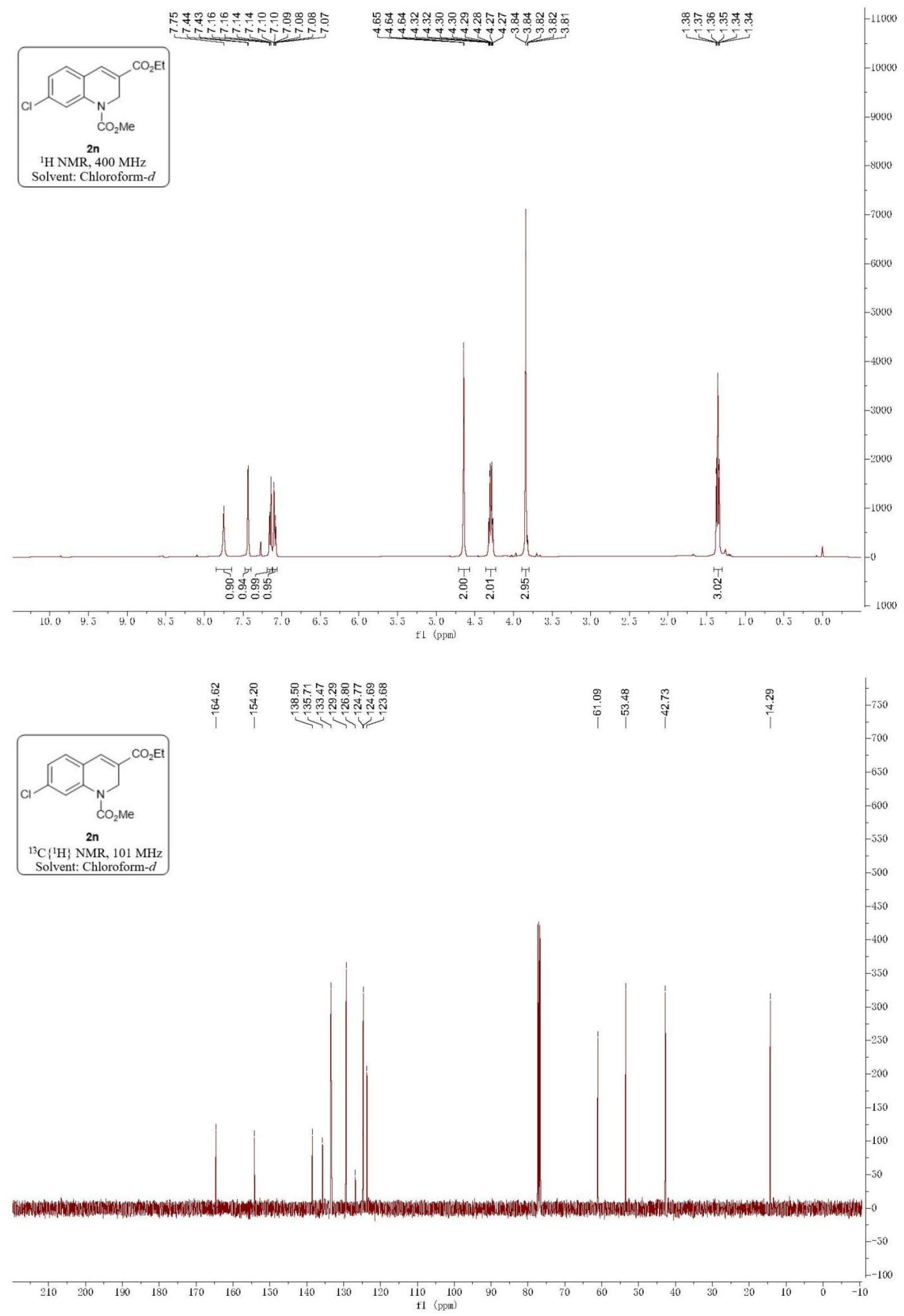

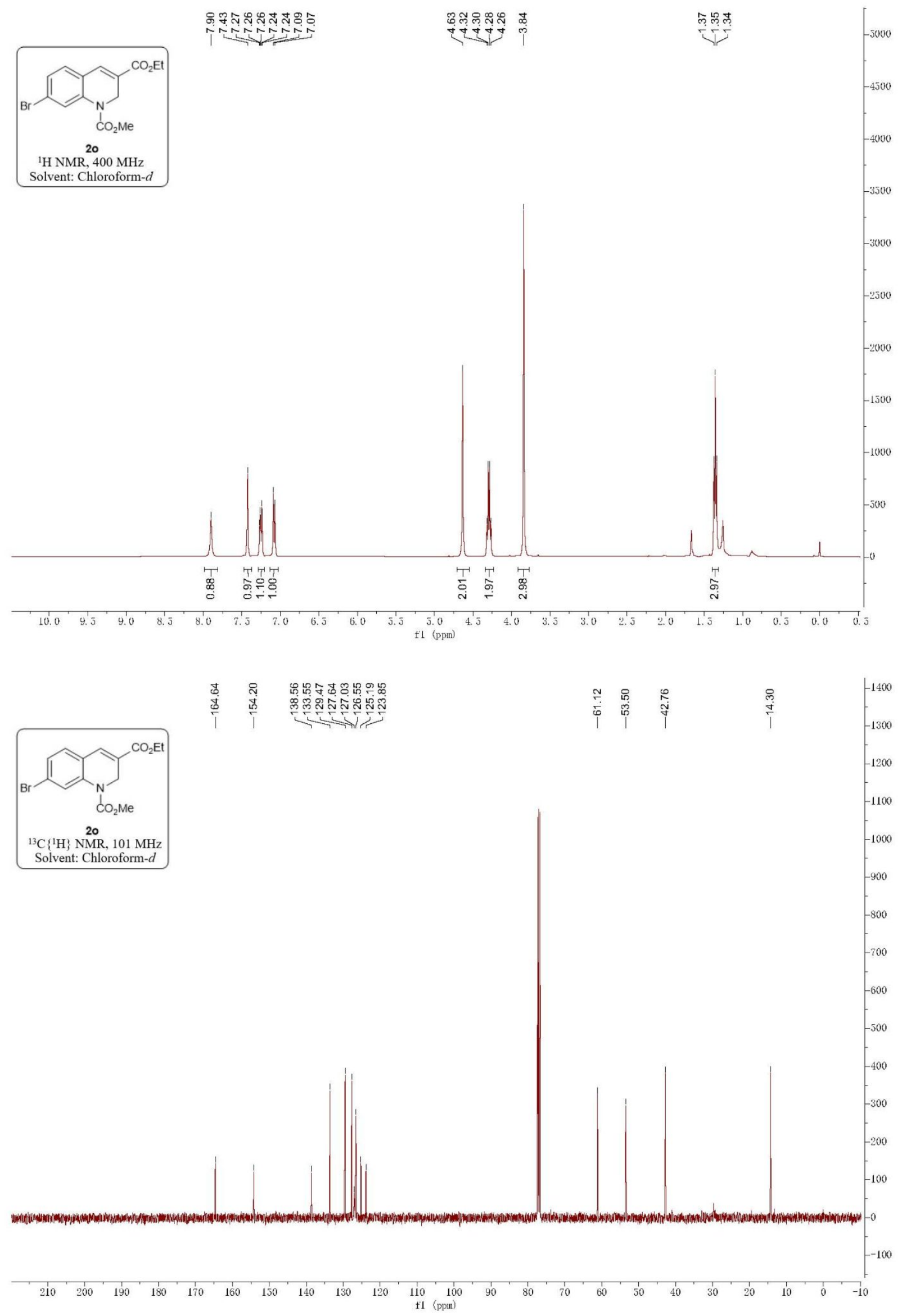

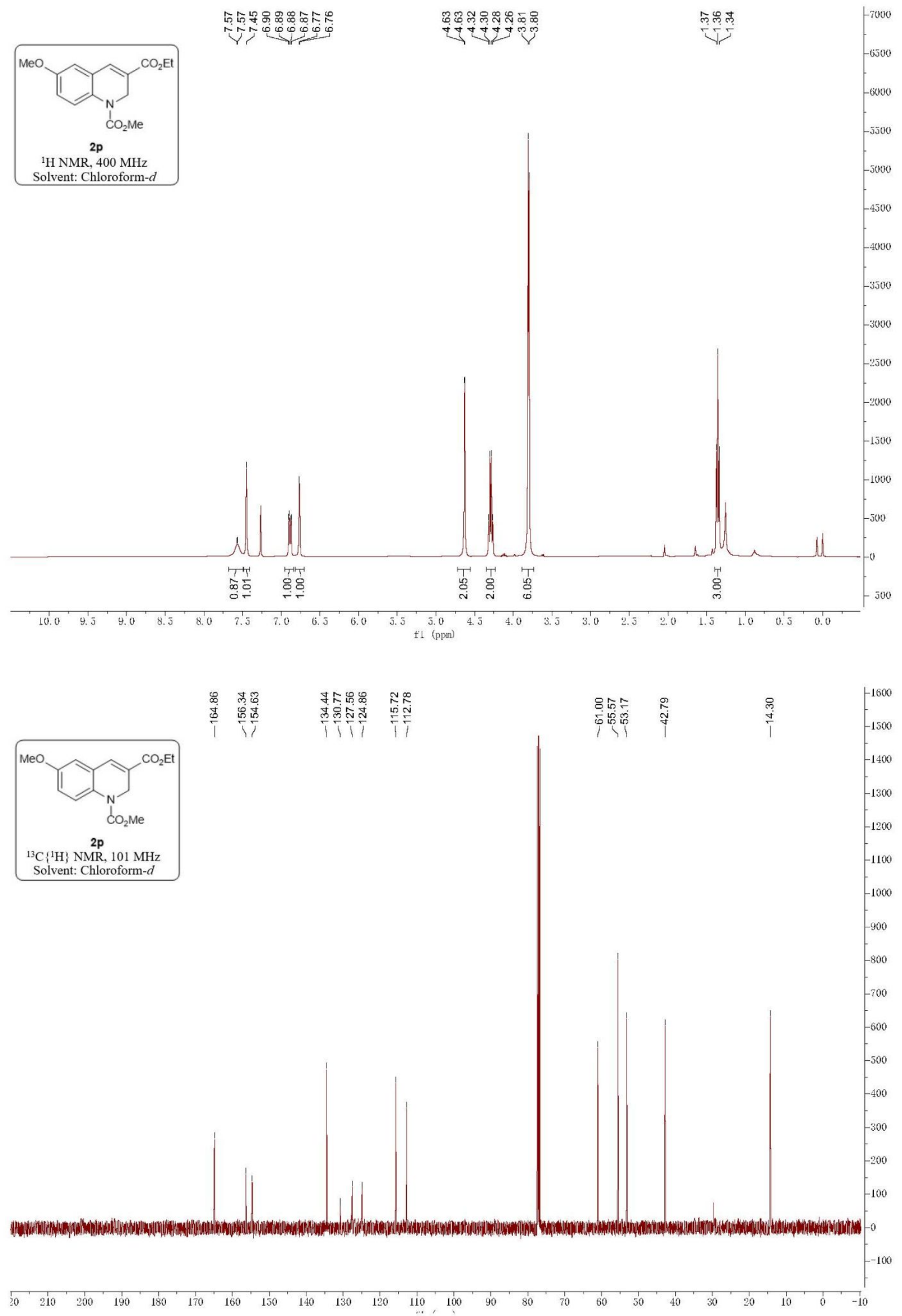


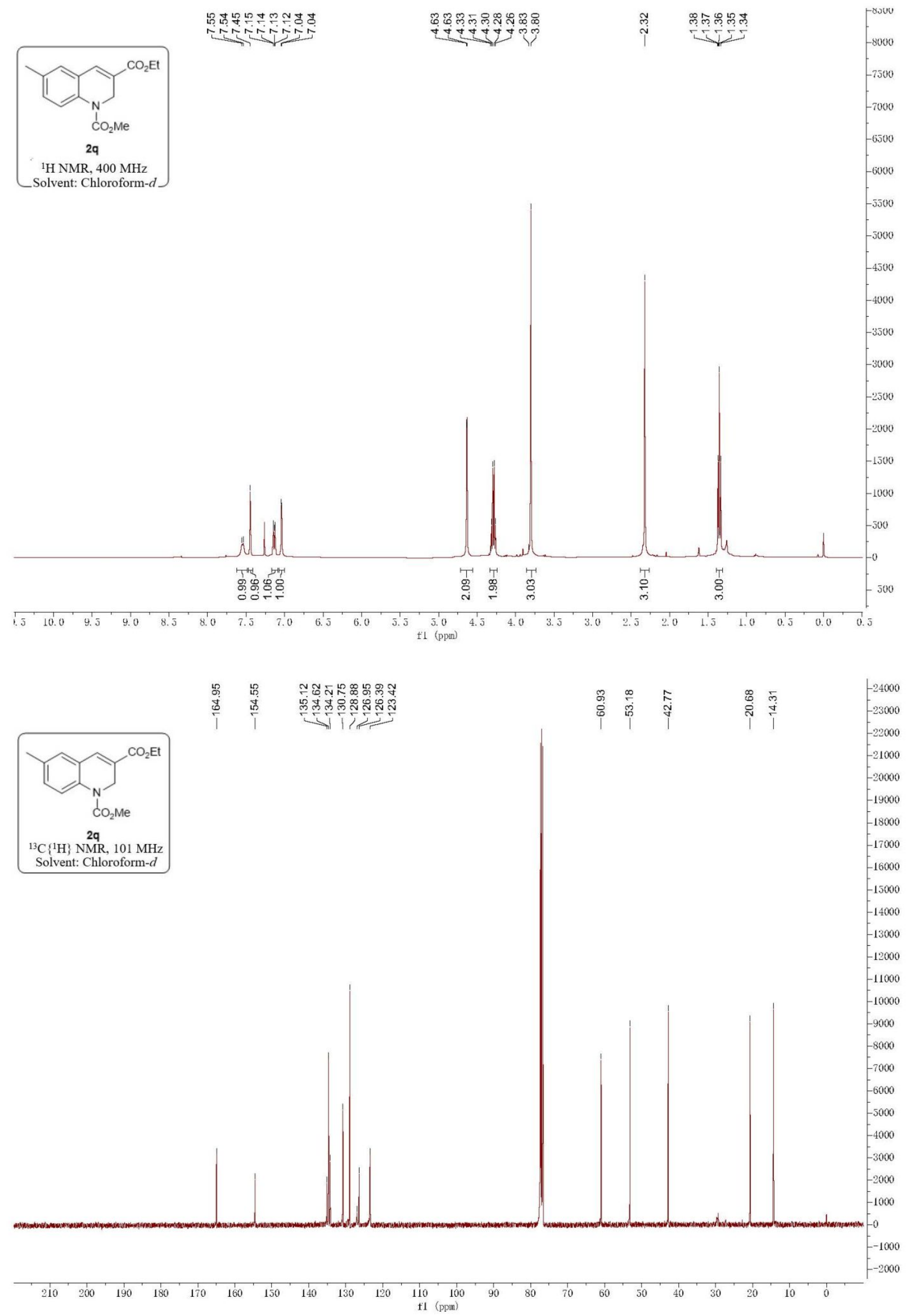




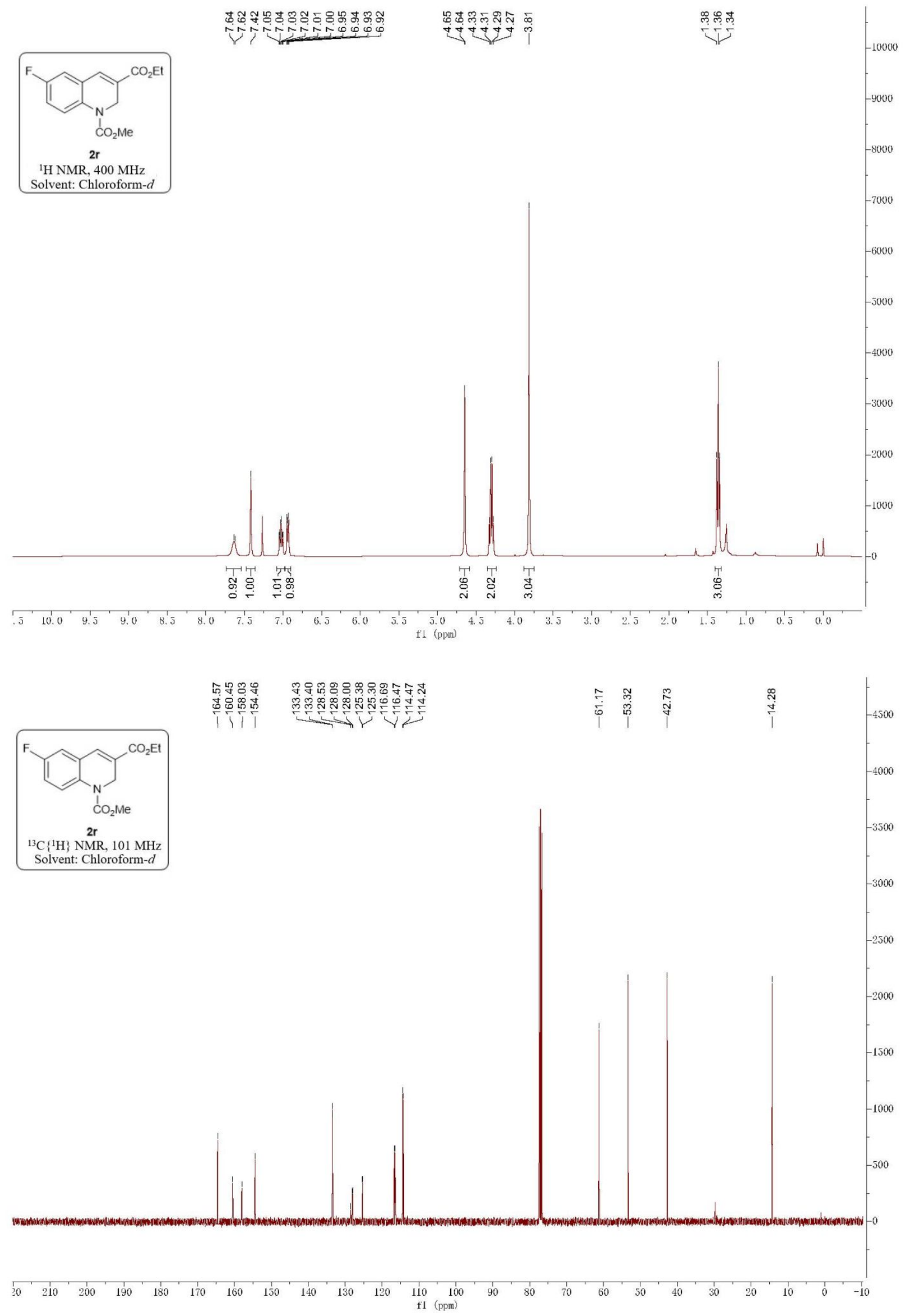




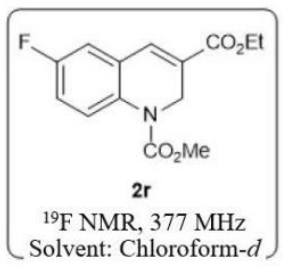

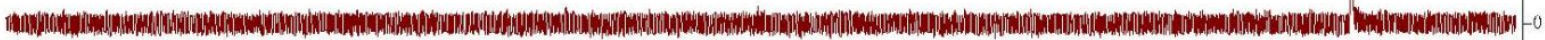

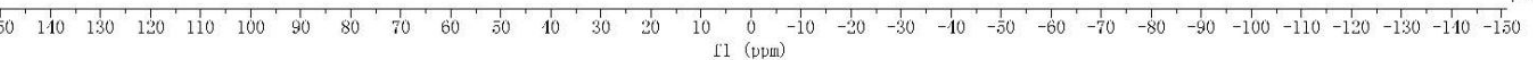

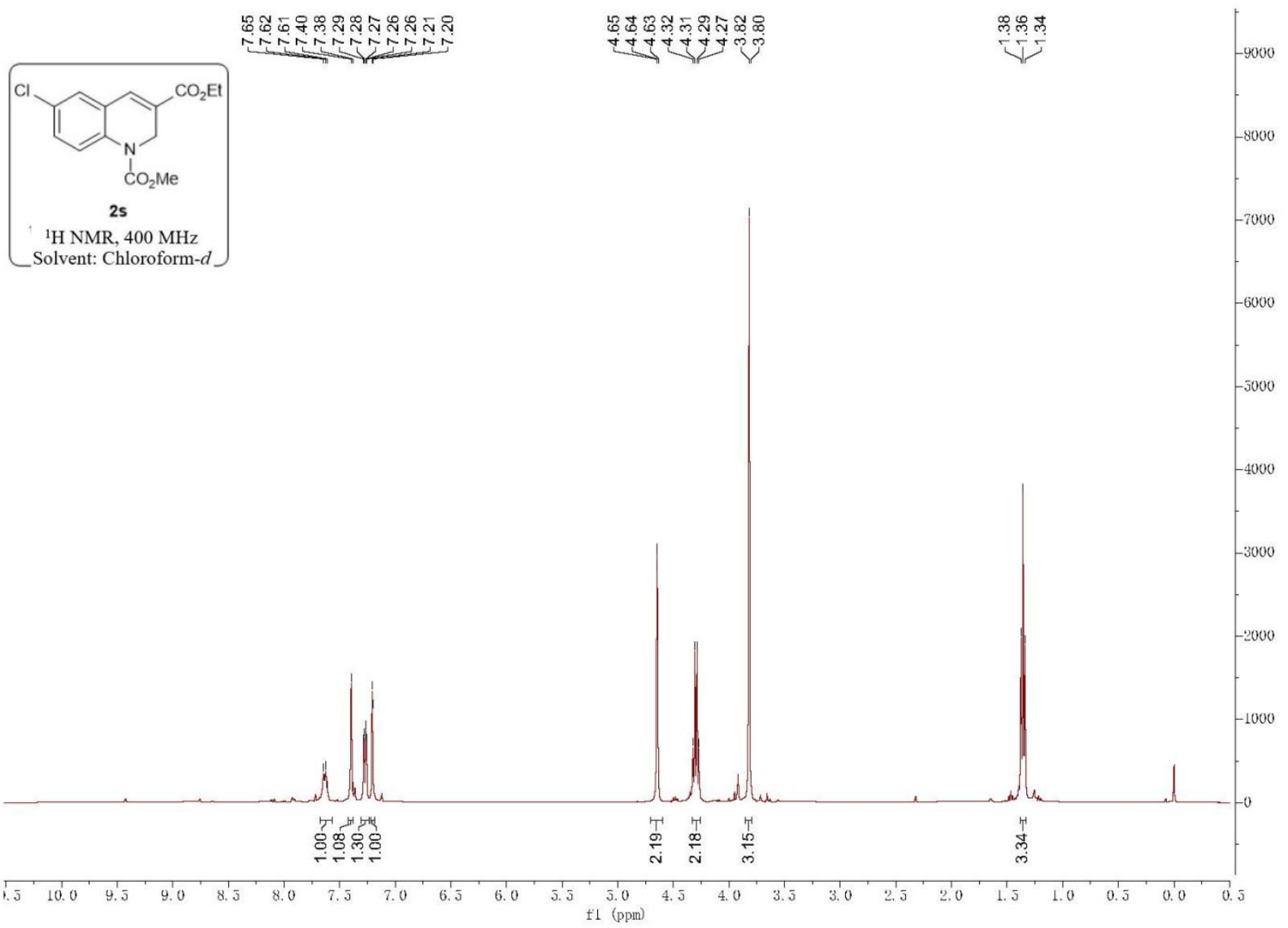




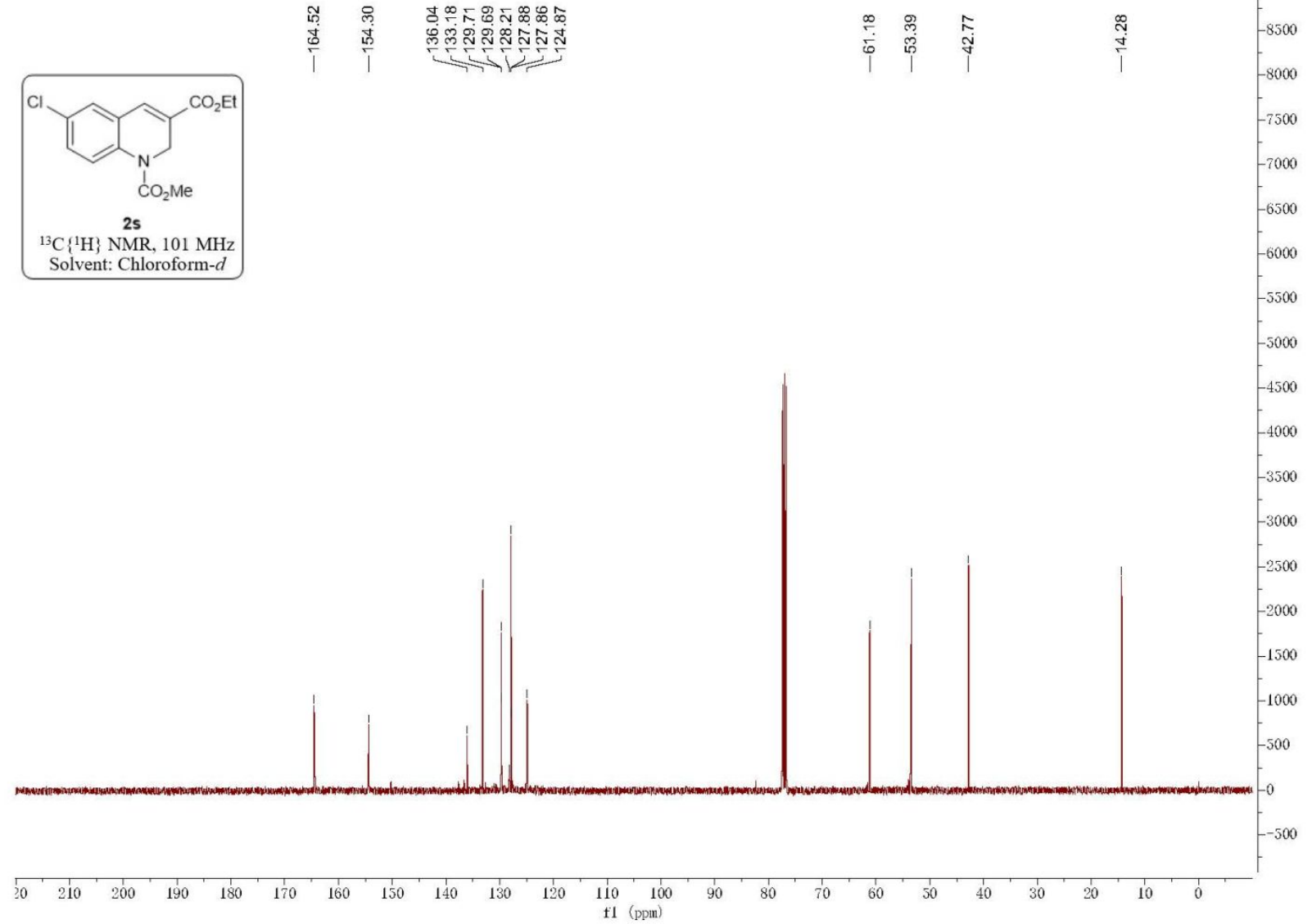

立

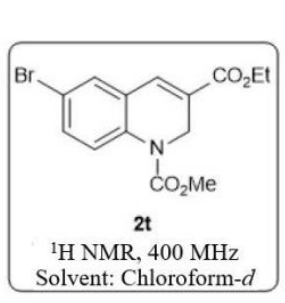

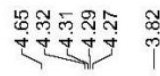

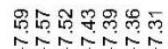

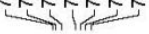

岇

-2800
$-u n u s$

Solvent: Chloroform- $d$

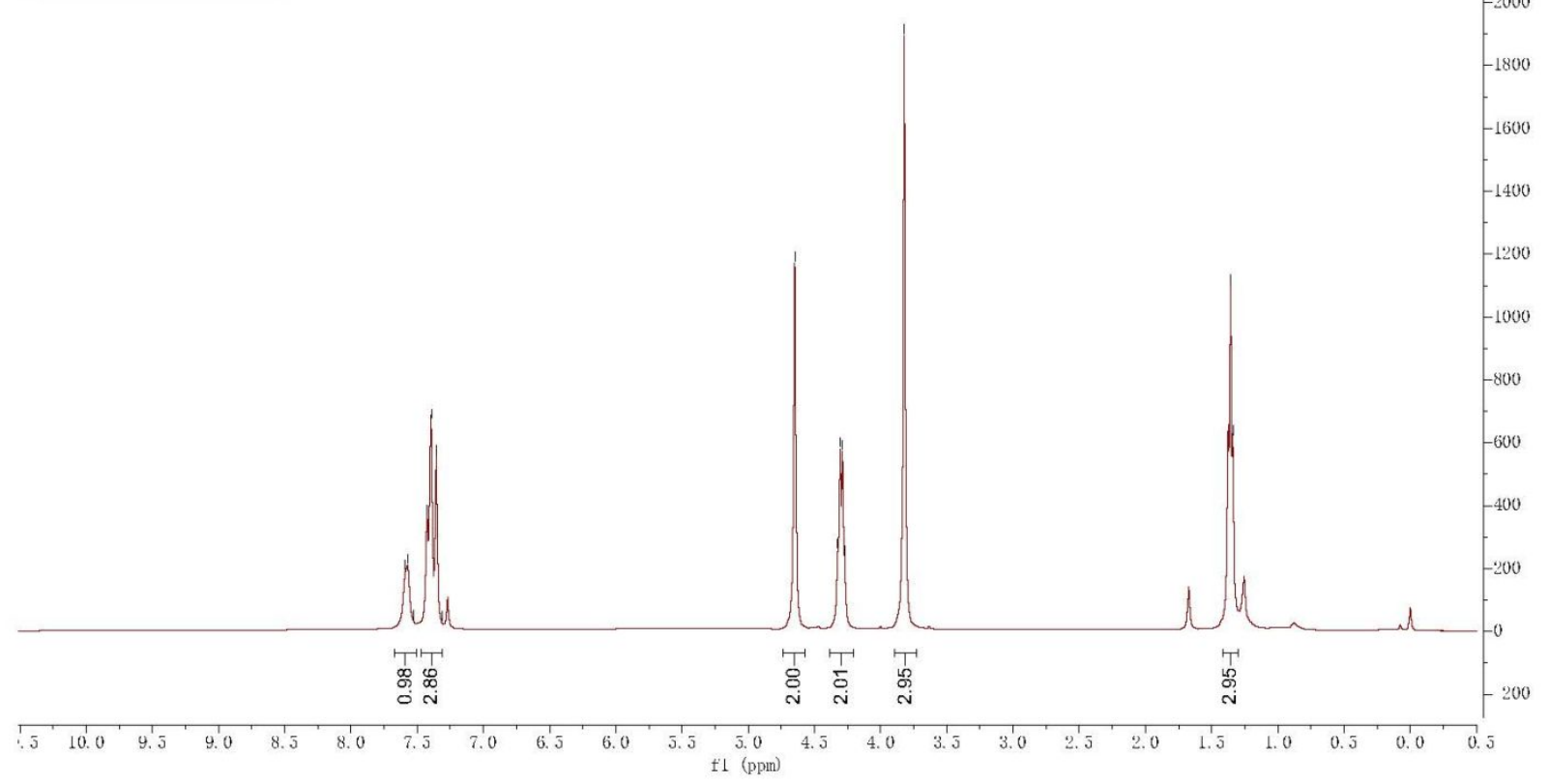



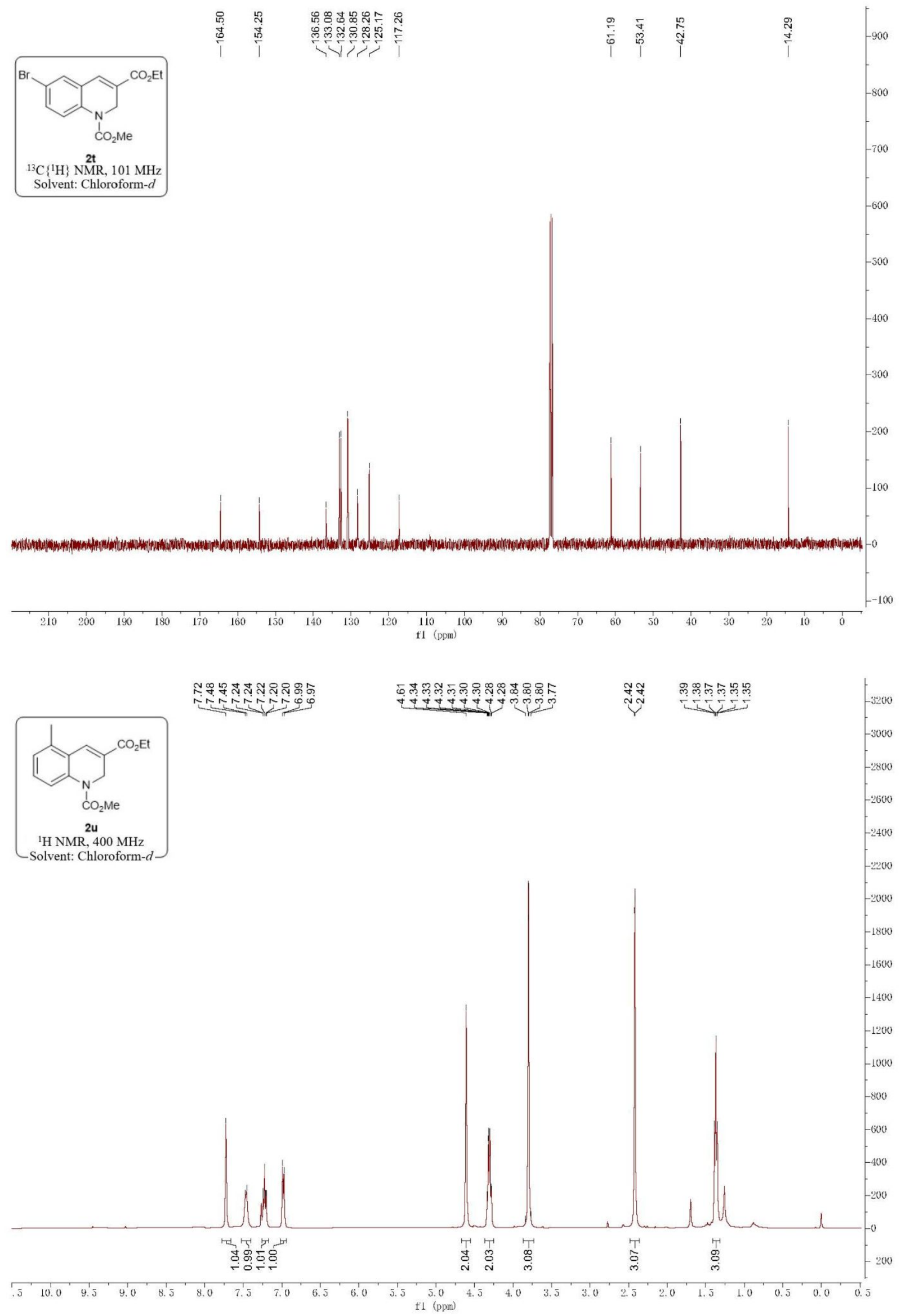


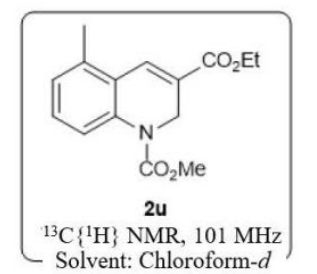

$$
\text { की }
$$

ำ
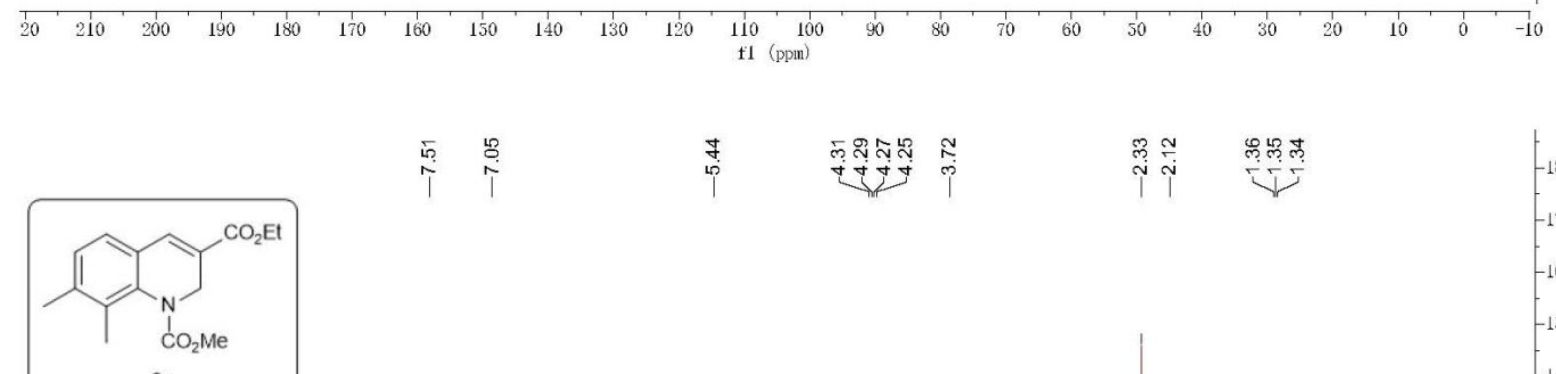

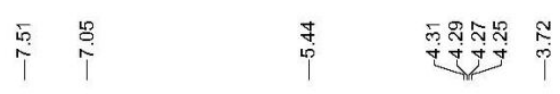

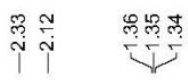

${ }^{1} \mathrm{H}$ NMR, $400 \mathrm{MHz}$

Solvent: Chloroform- $d$

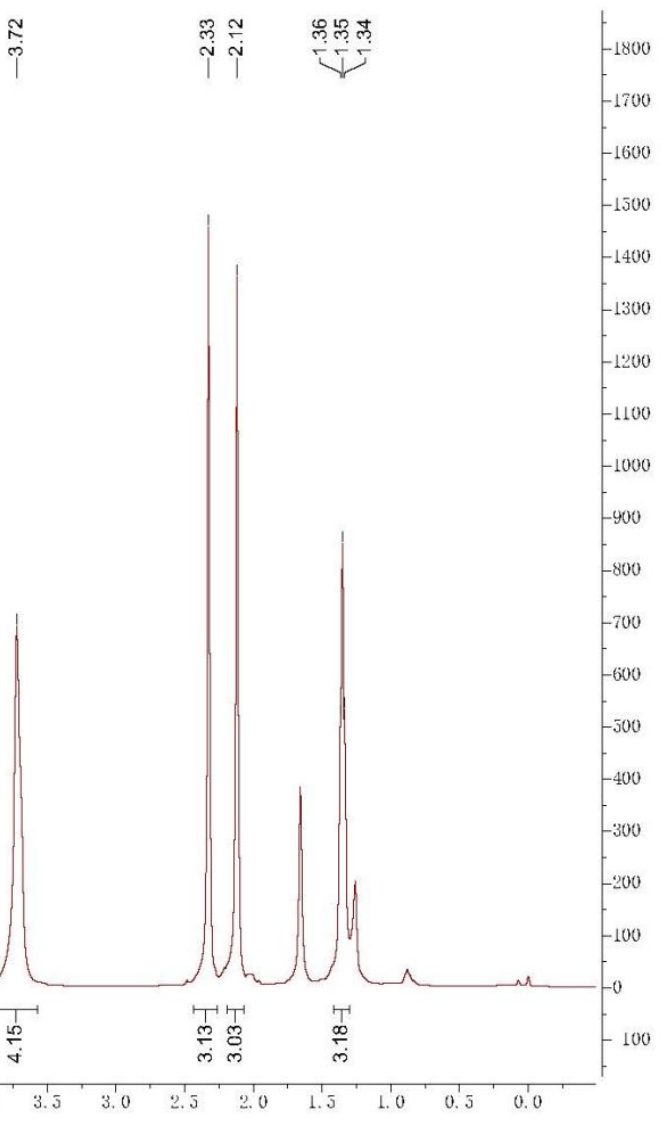




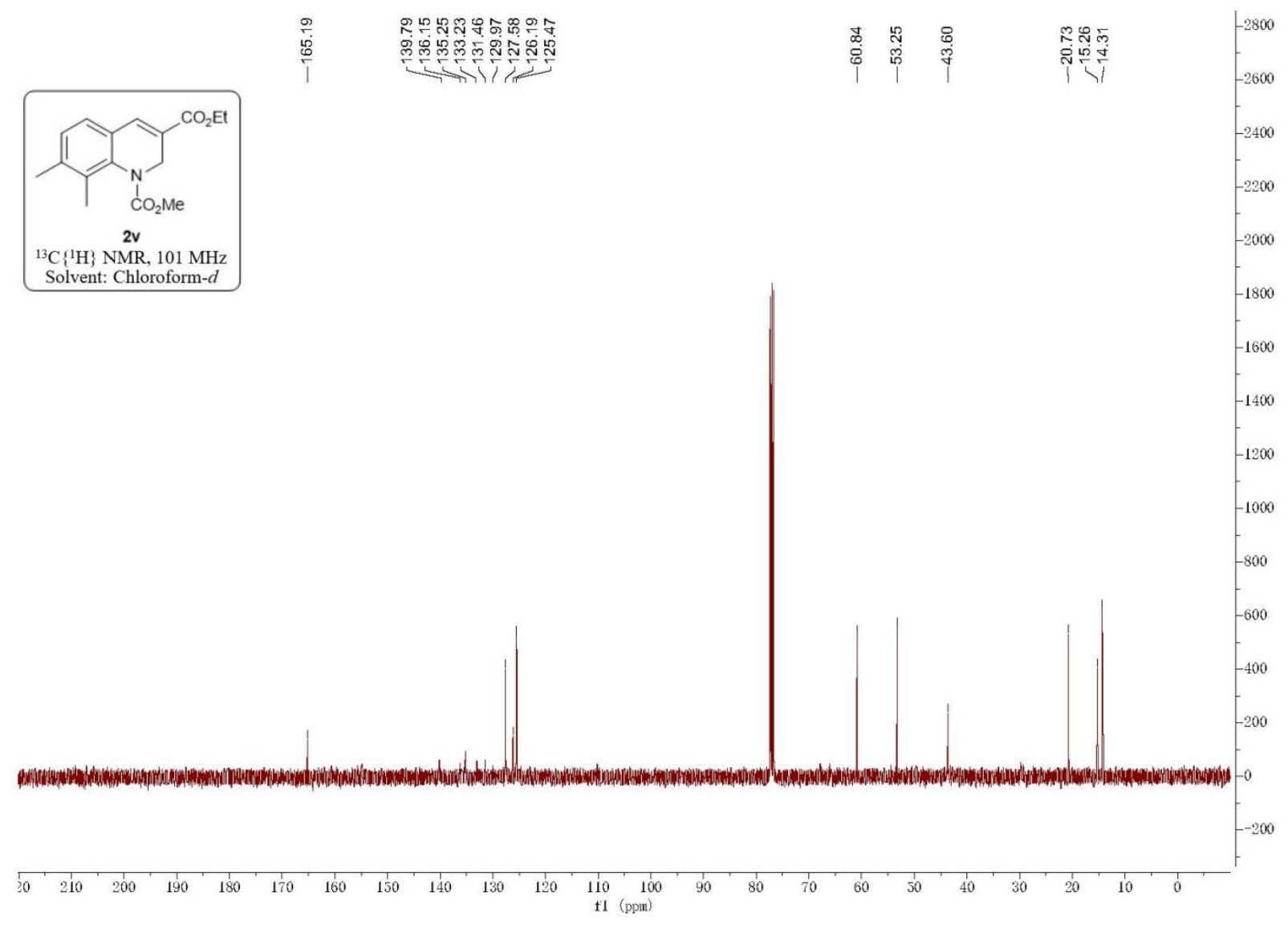


4.3. quinuclidine catalyzed intramolecular cyclization products 3
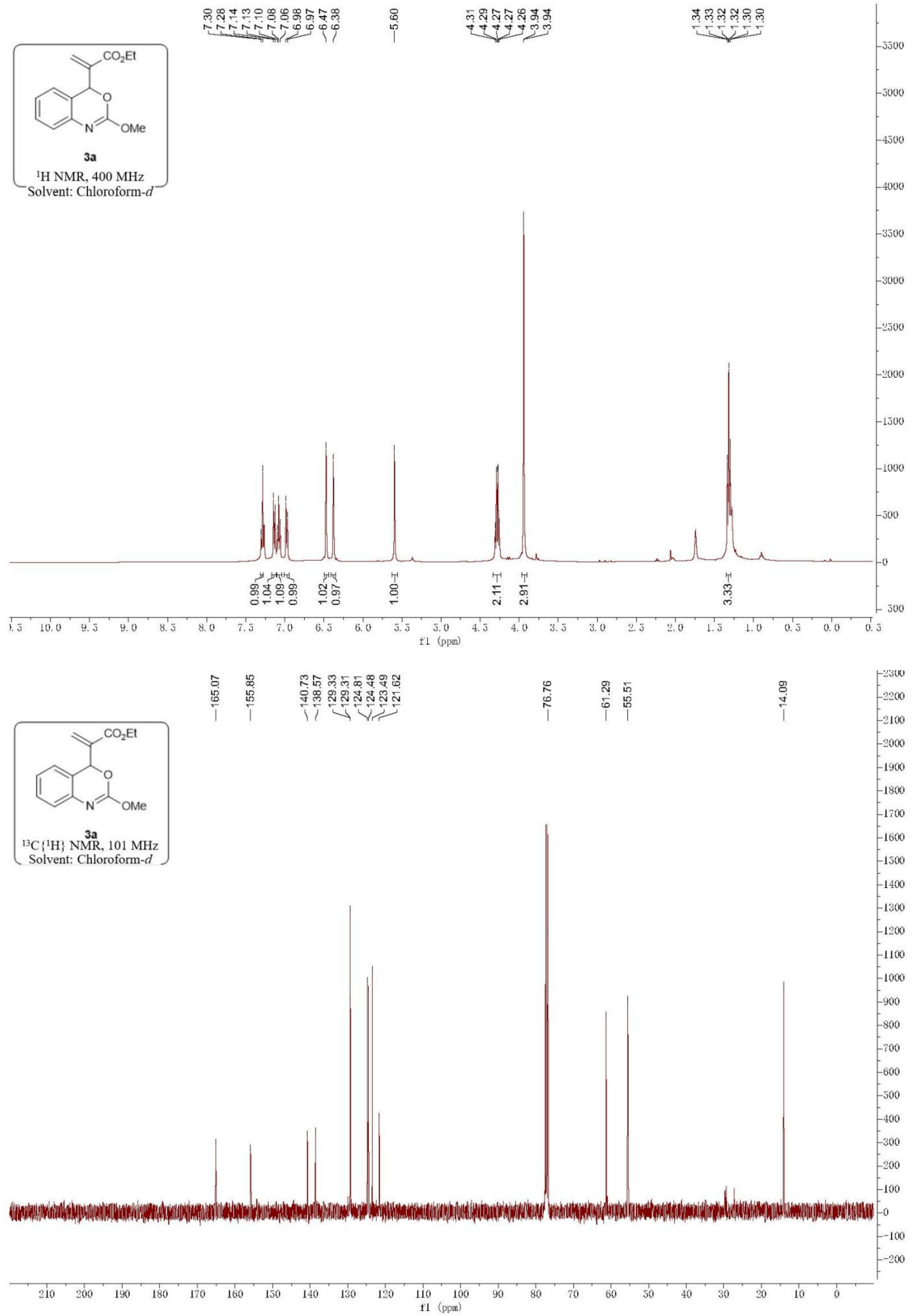

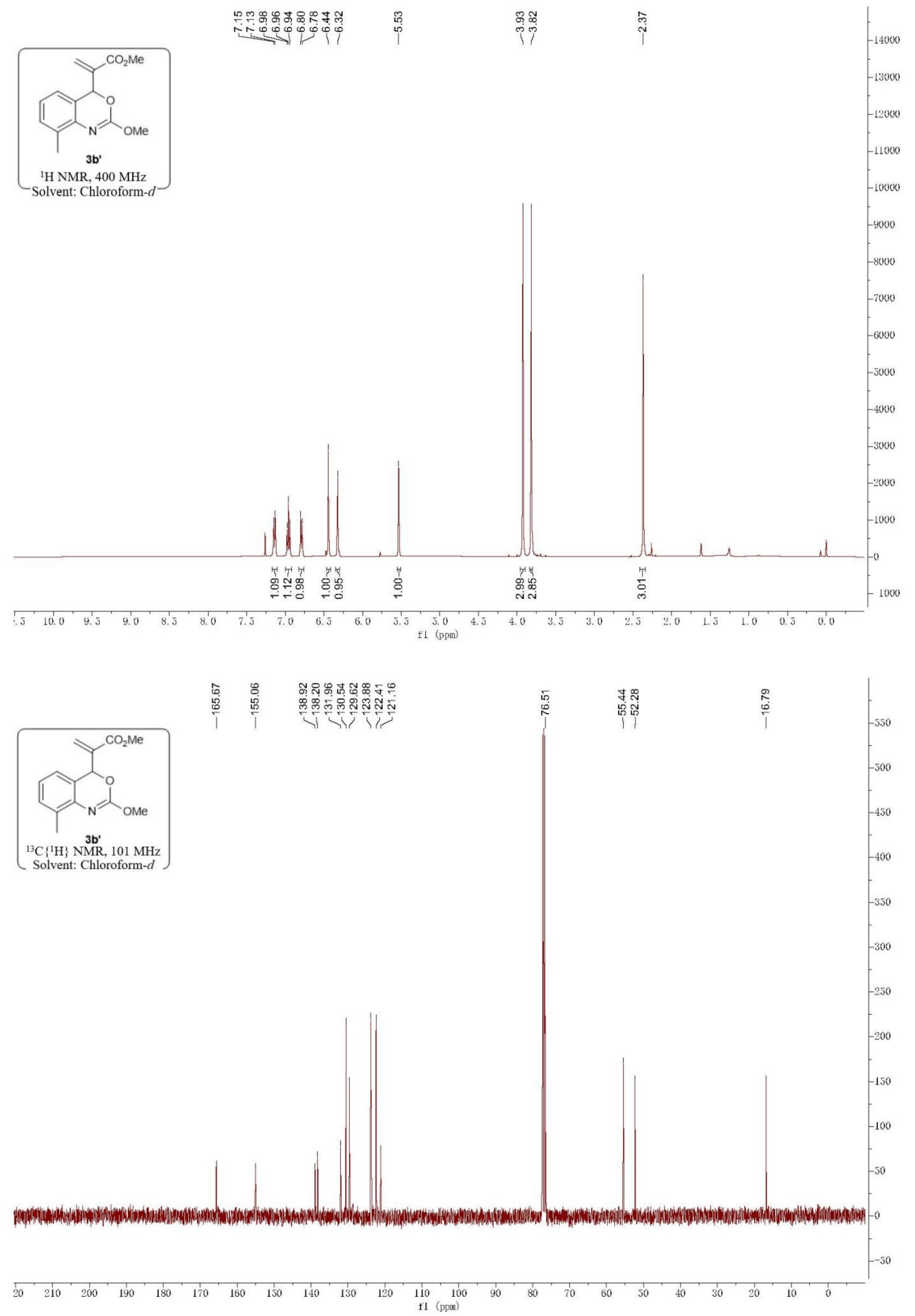

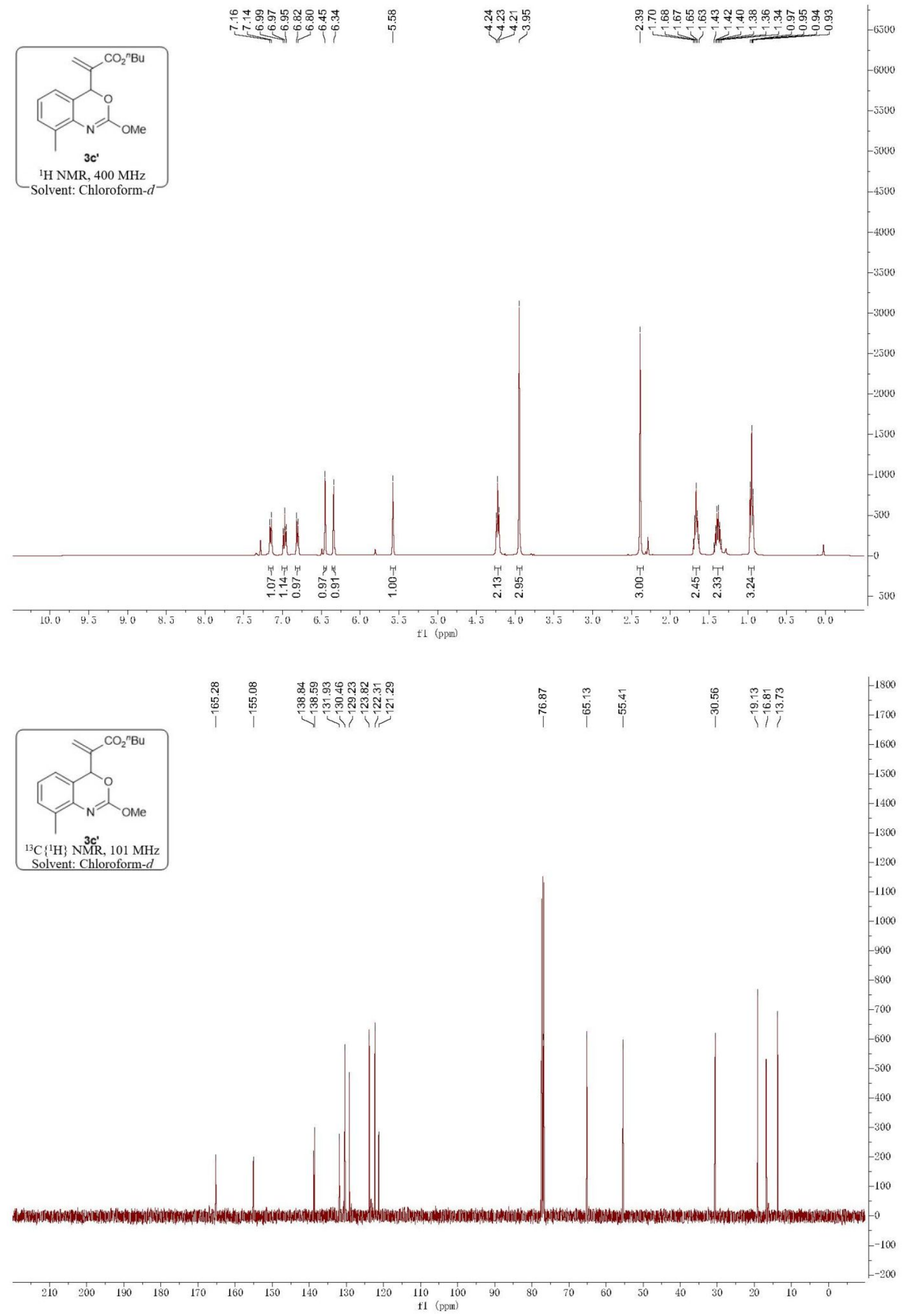

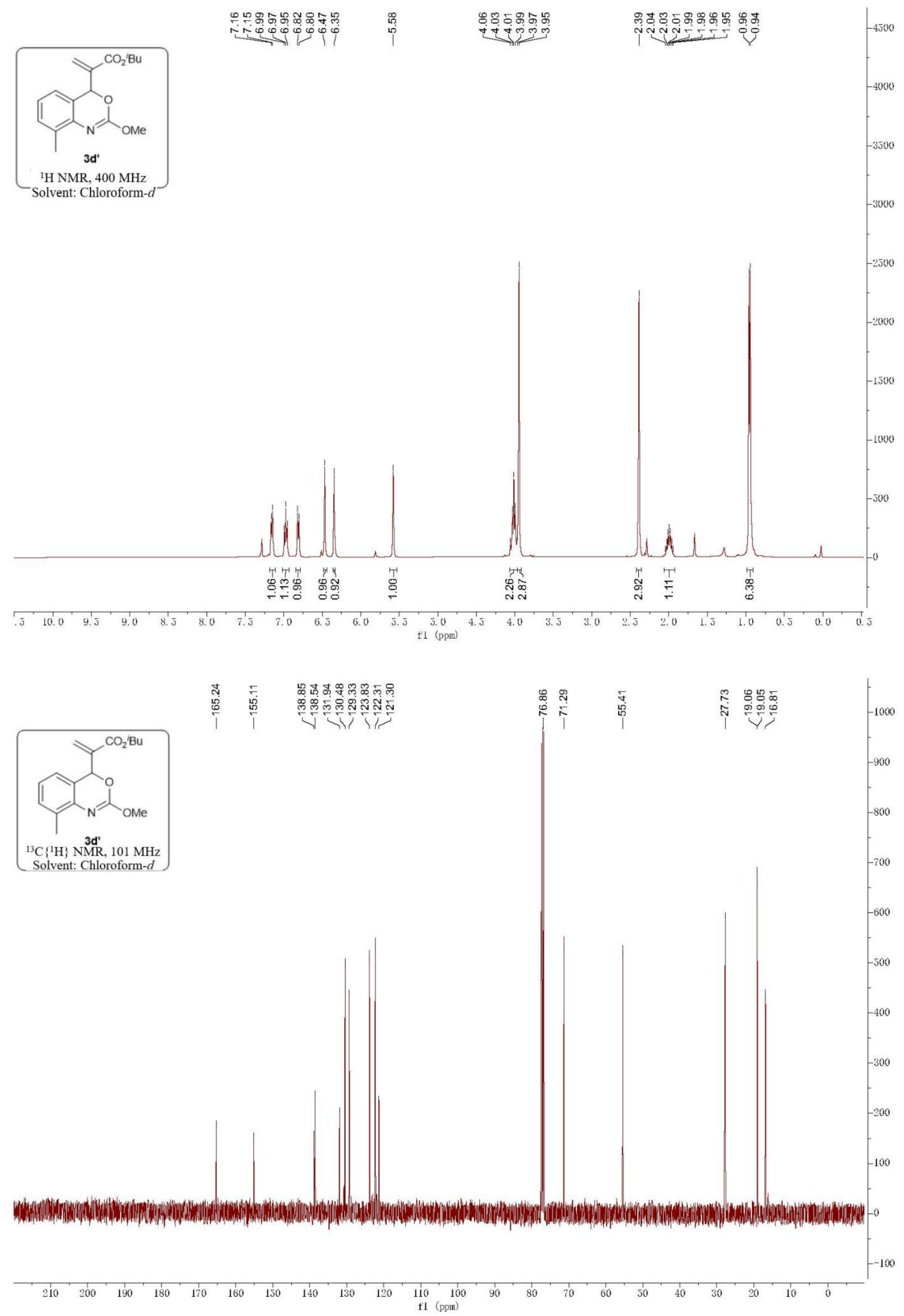

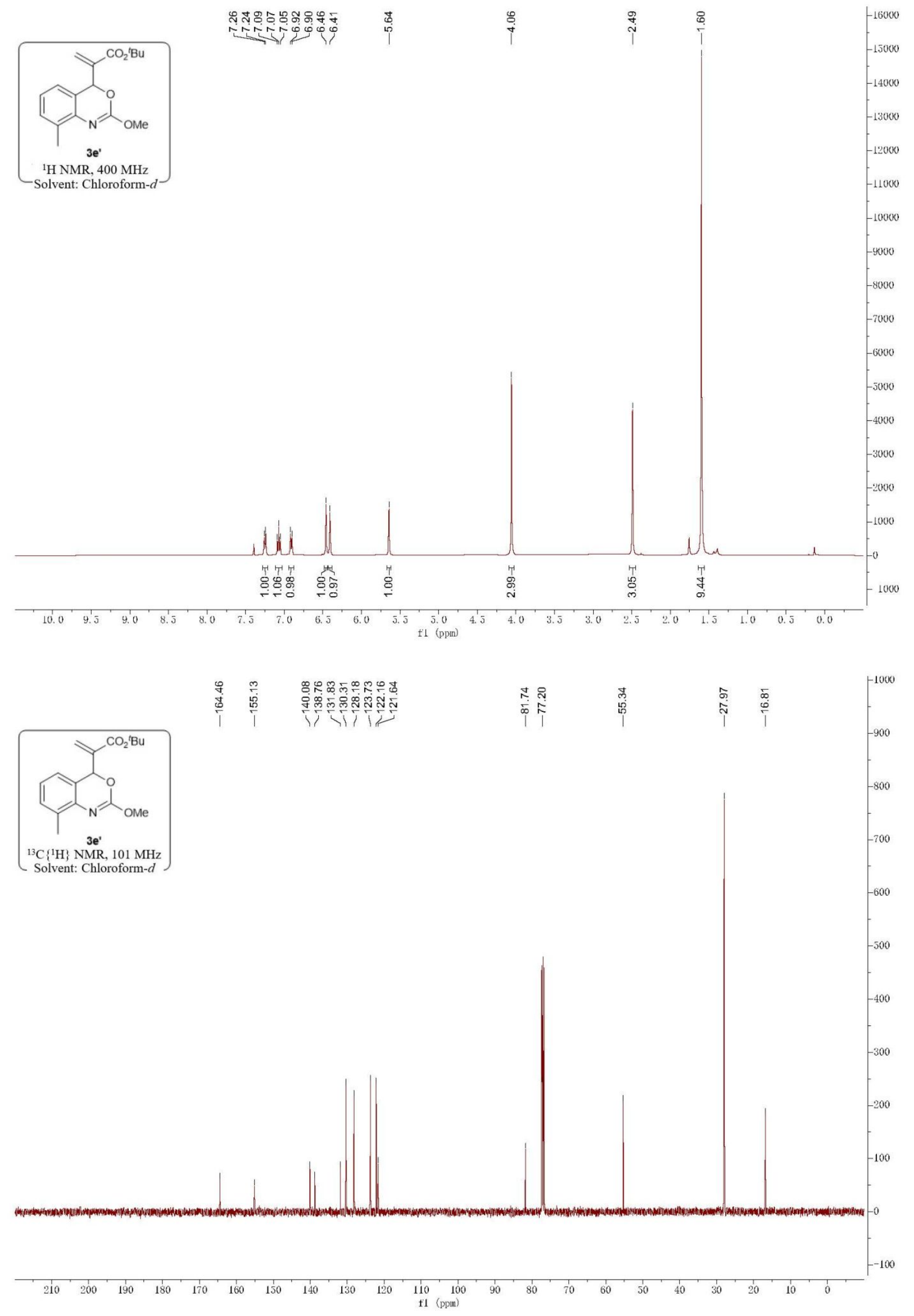

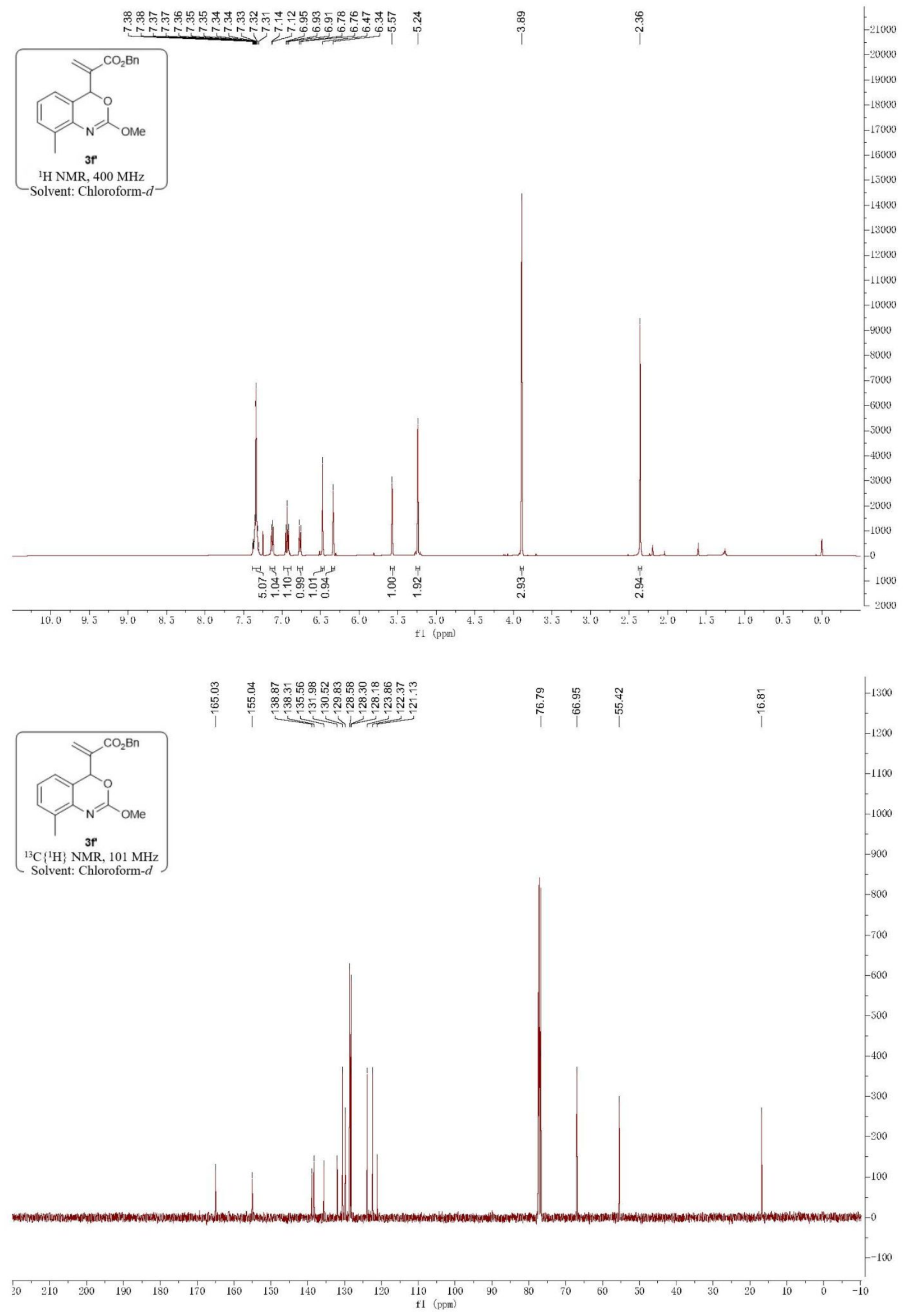


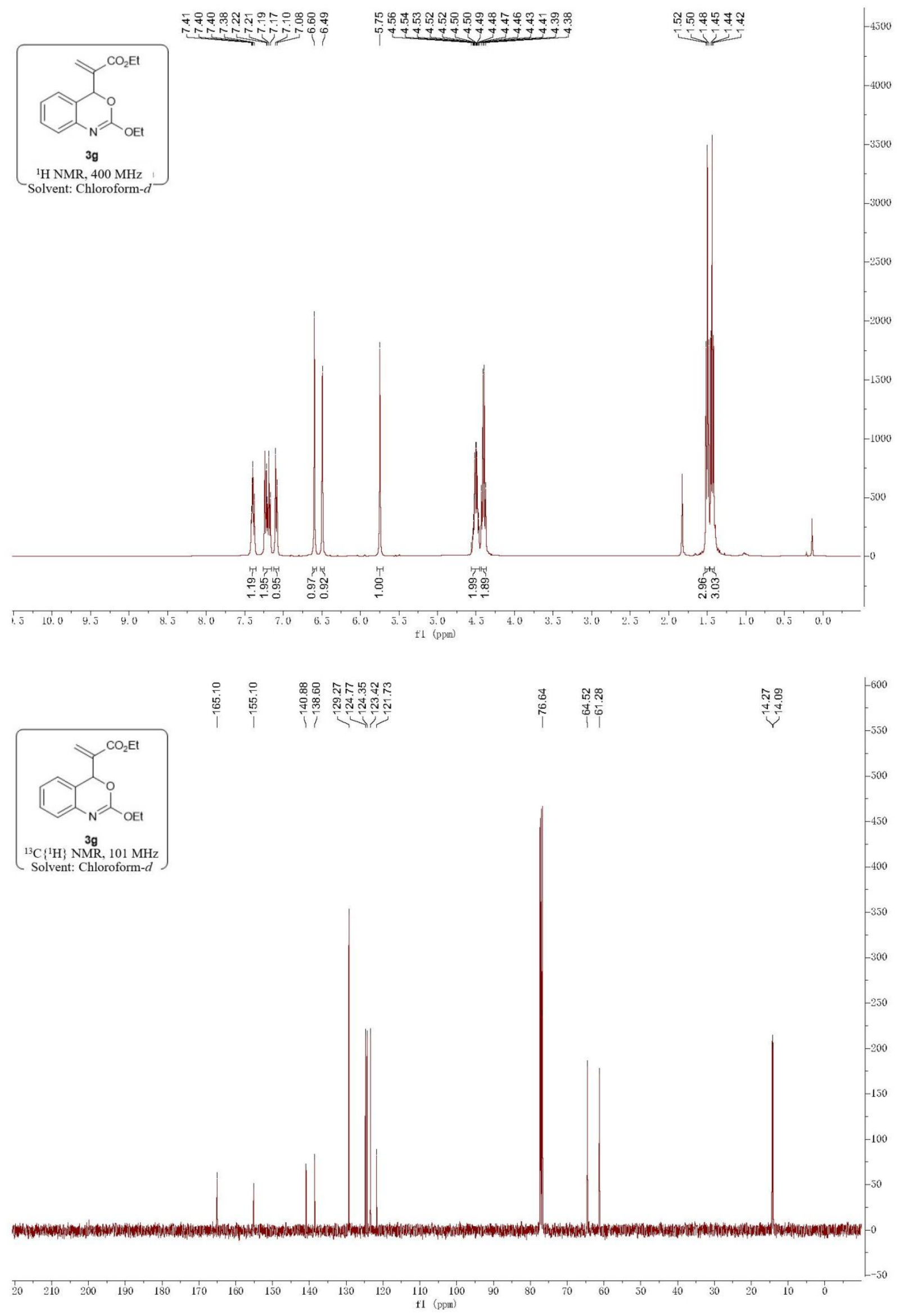




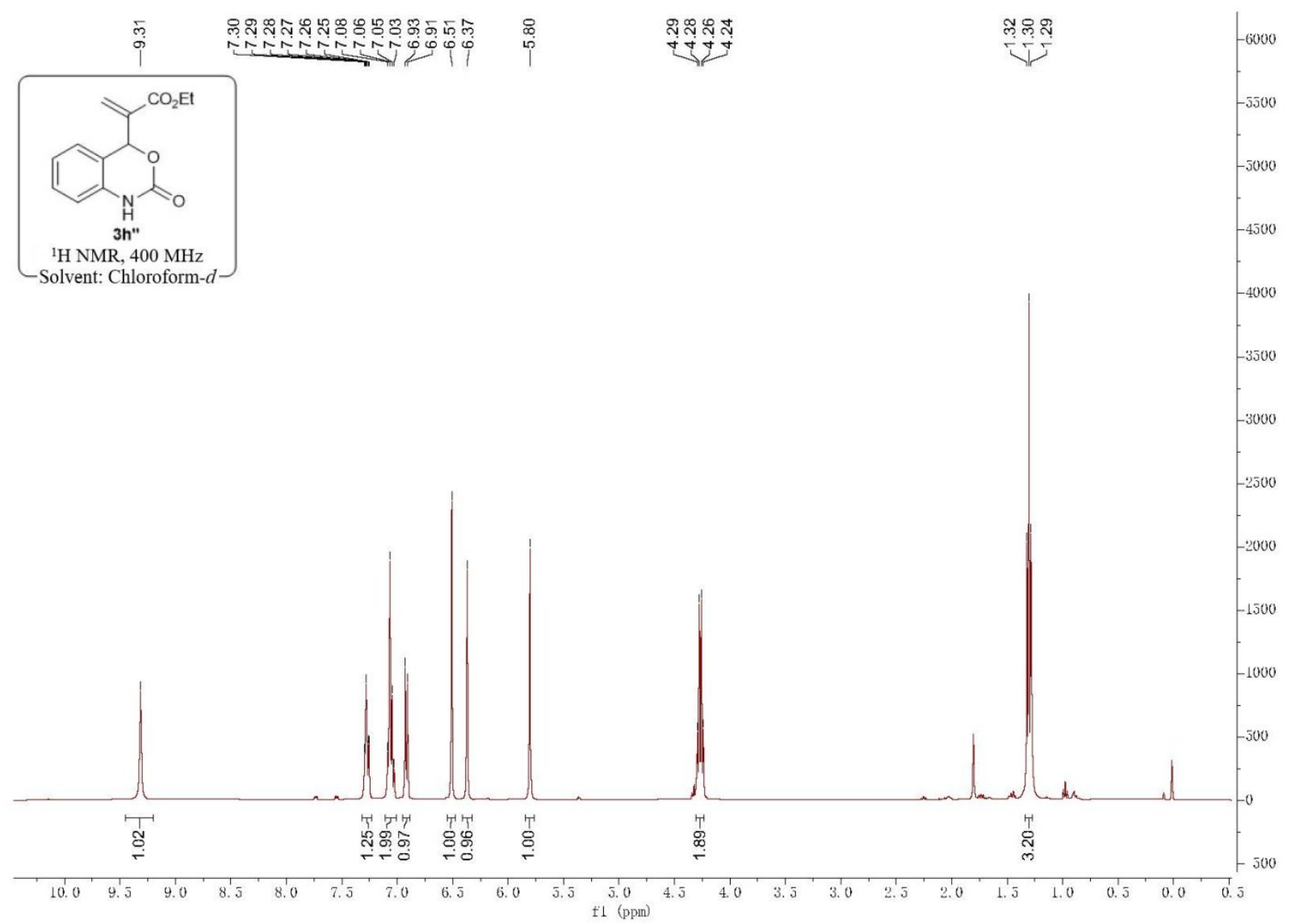

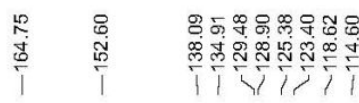

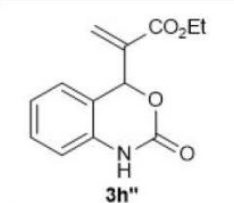

$\begin{array}{ll}\frac{1}{n} & \frac{1}{2}\end{array}$

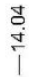

450

( $\left.{ }^{1} \mathrm{H}\right\} \mathrm{NMR}, 101 \mathrm{MHz}$

Solvent: Chloroform- $d$ 

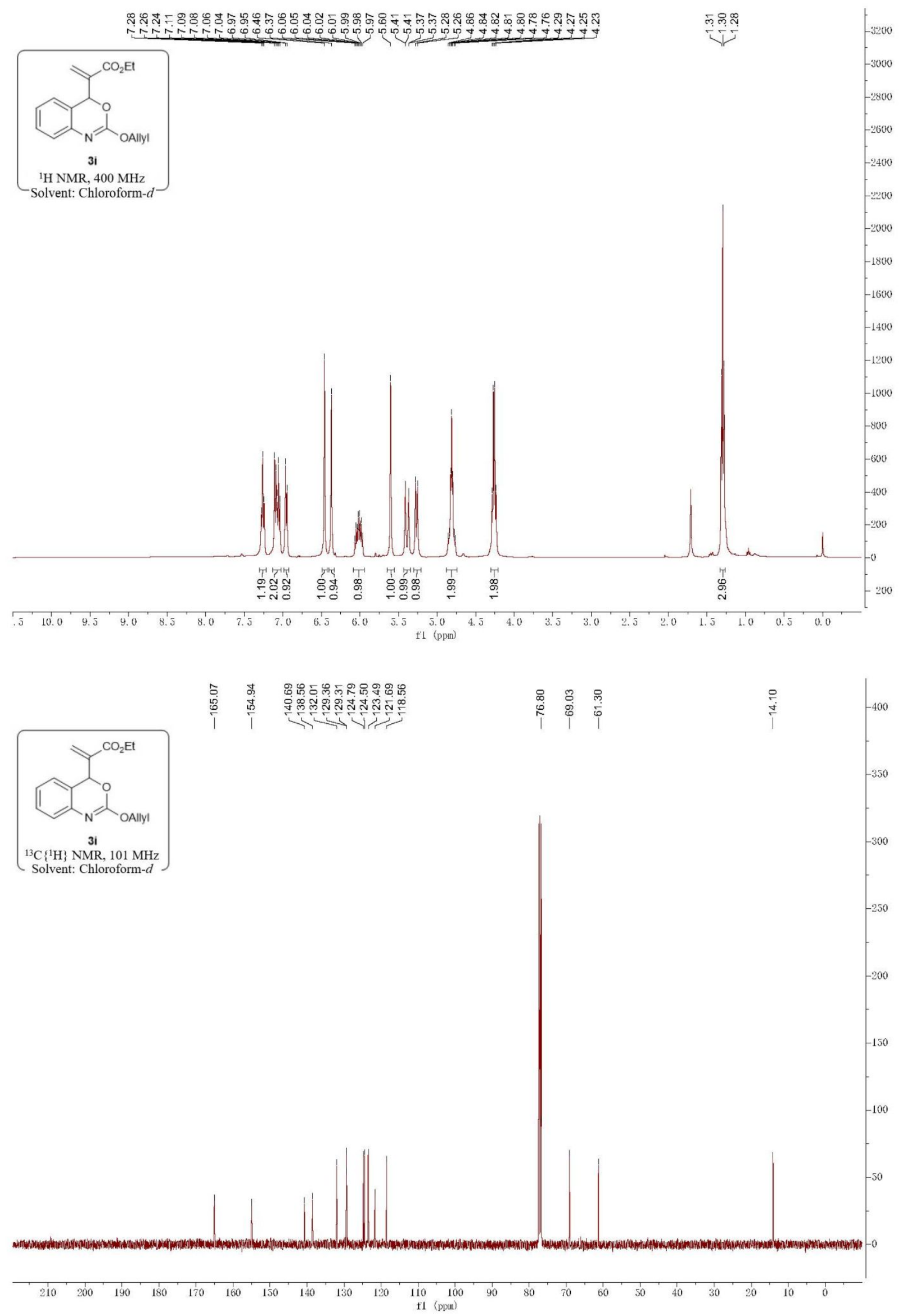

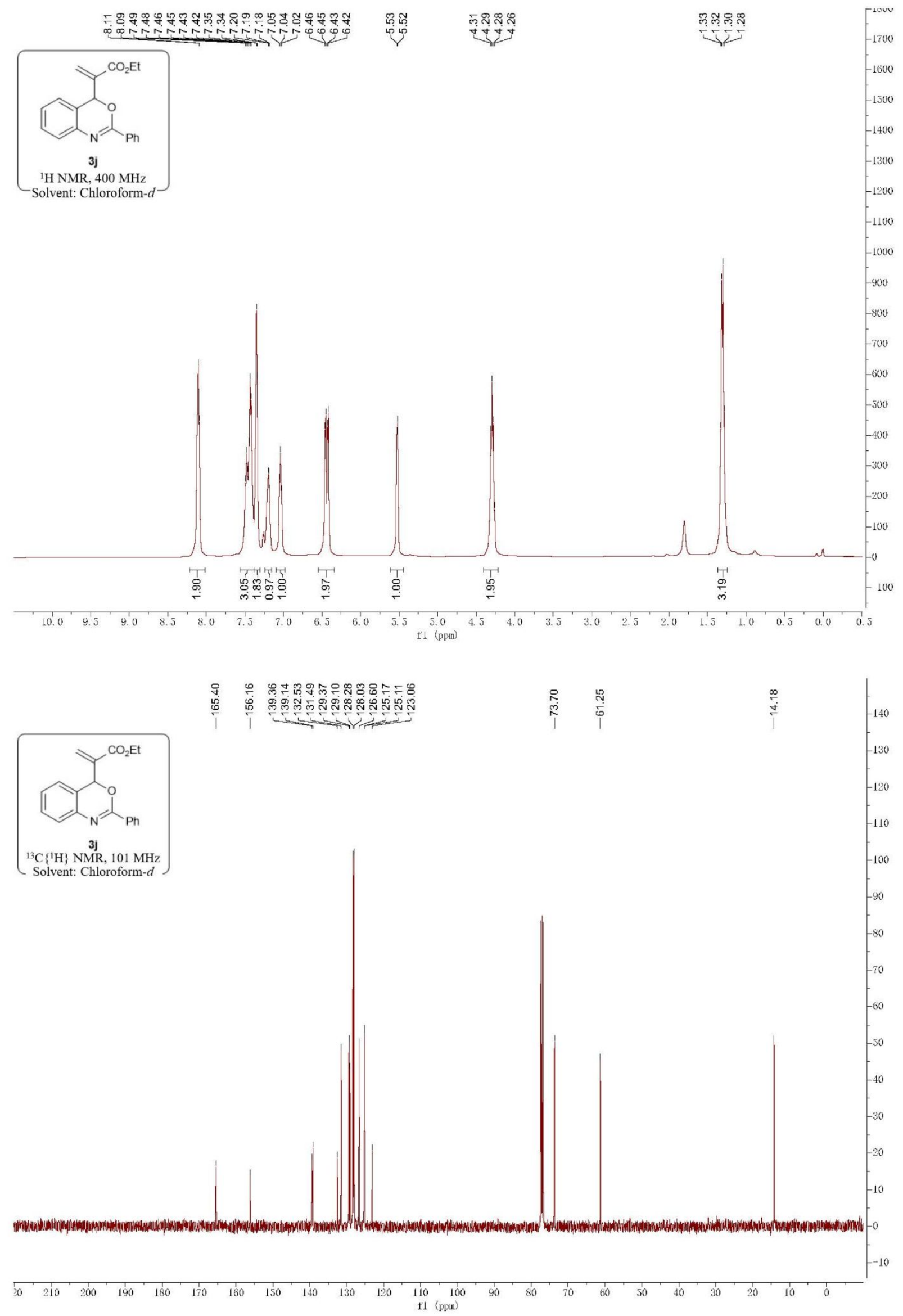

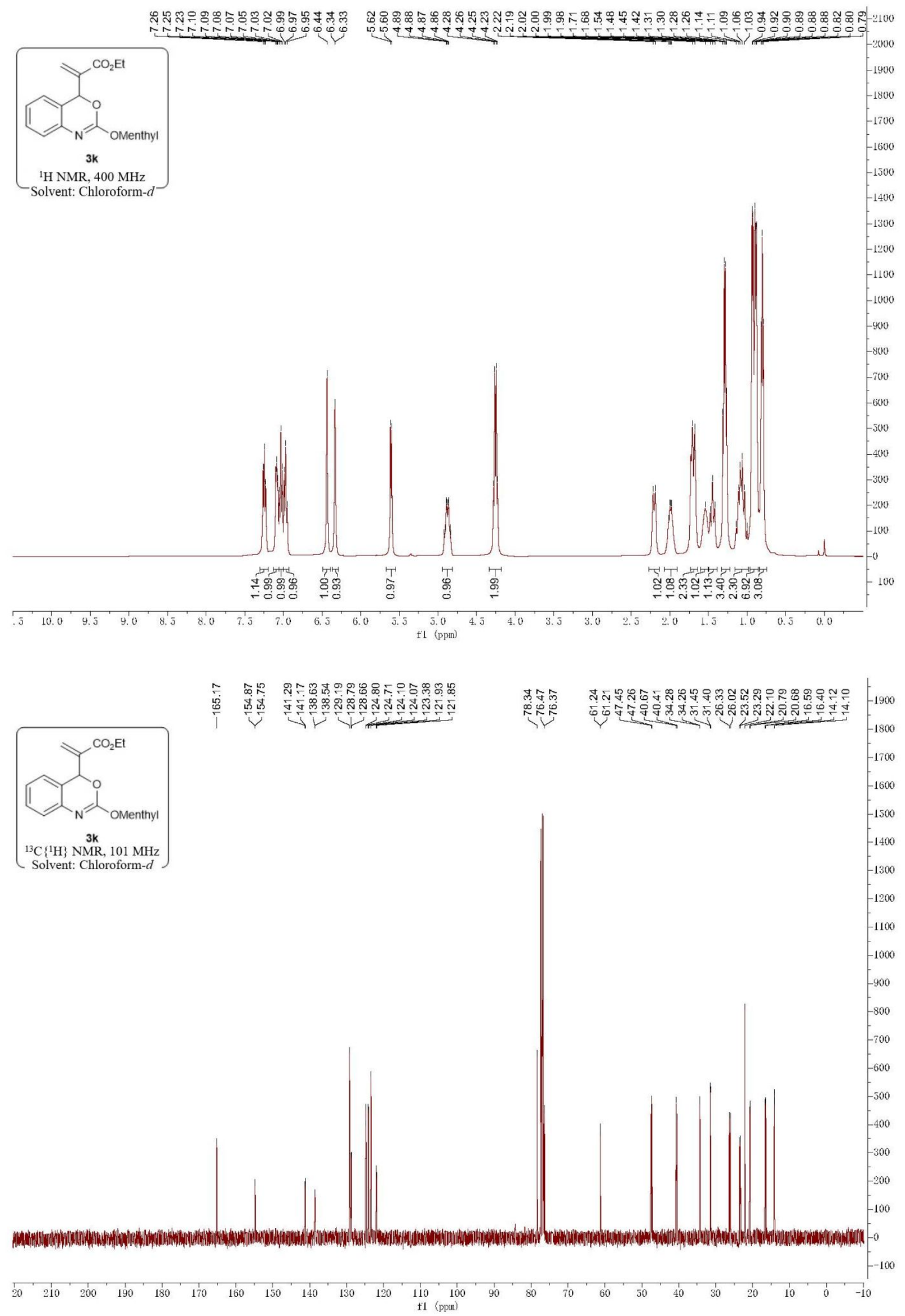


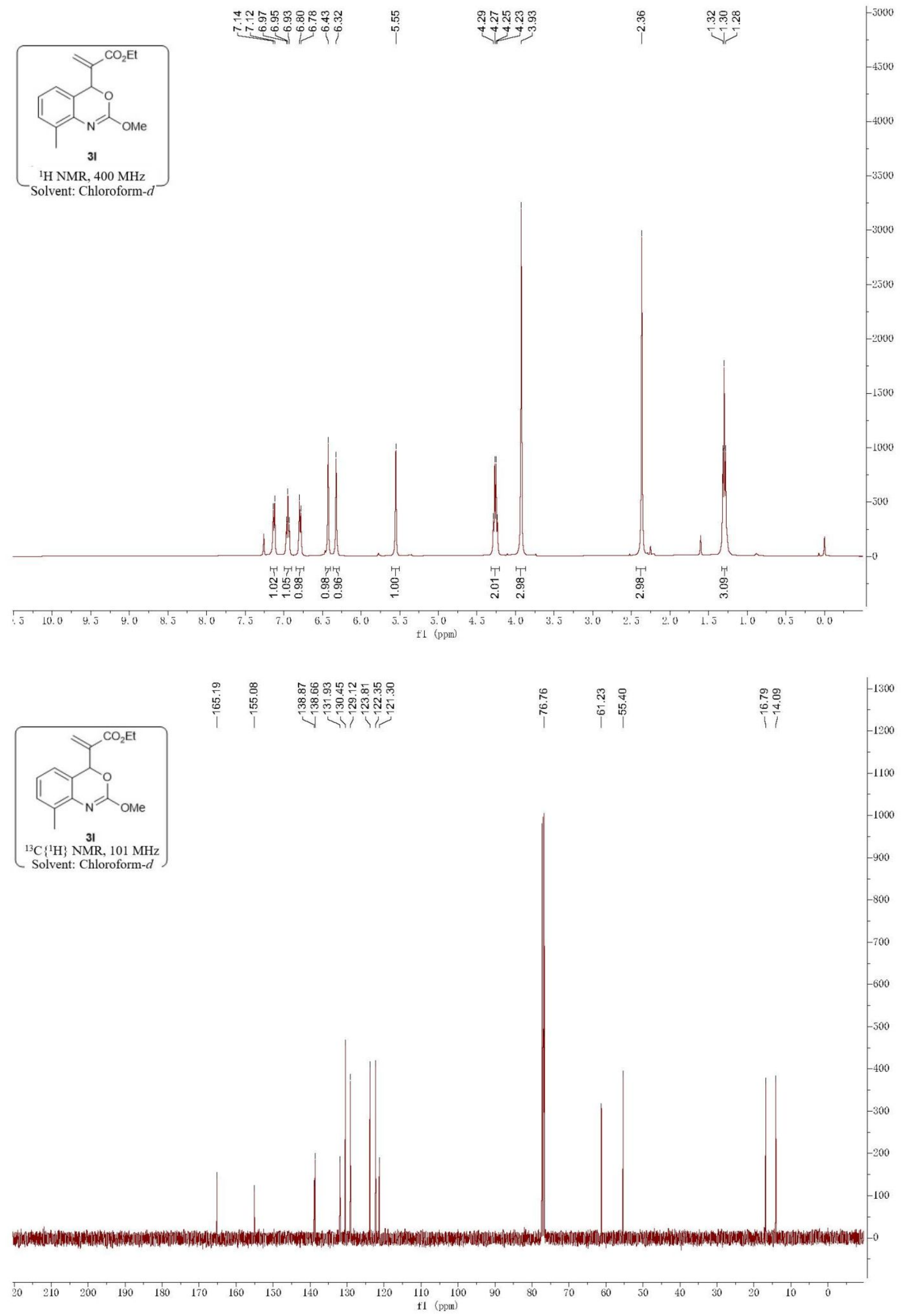



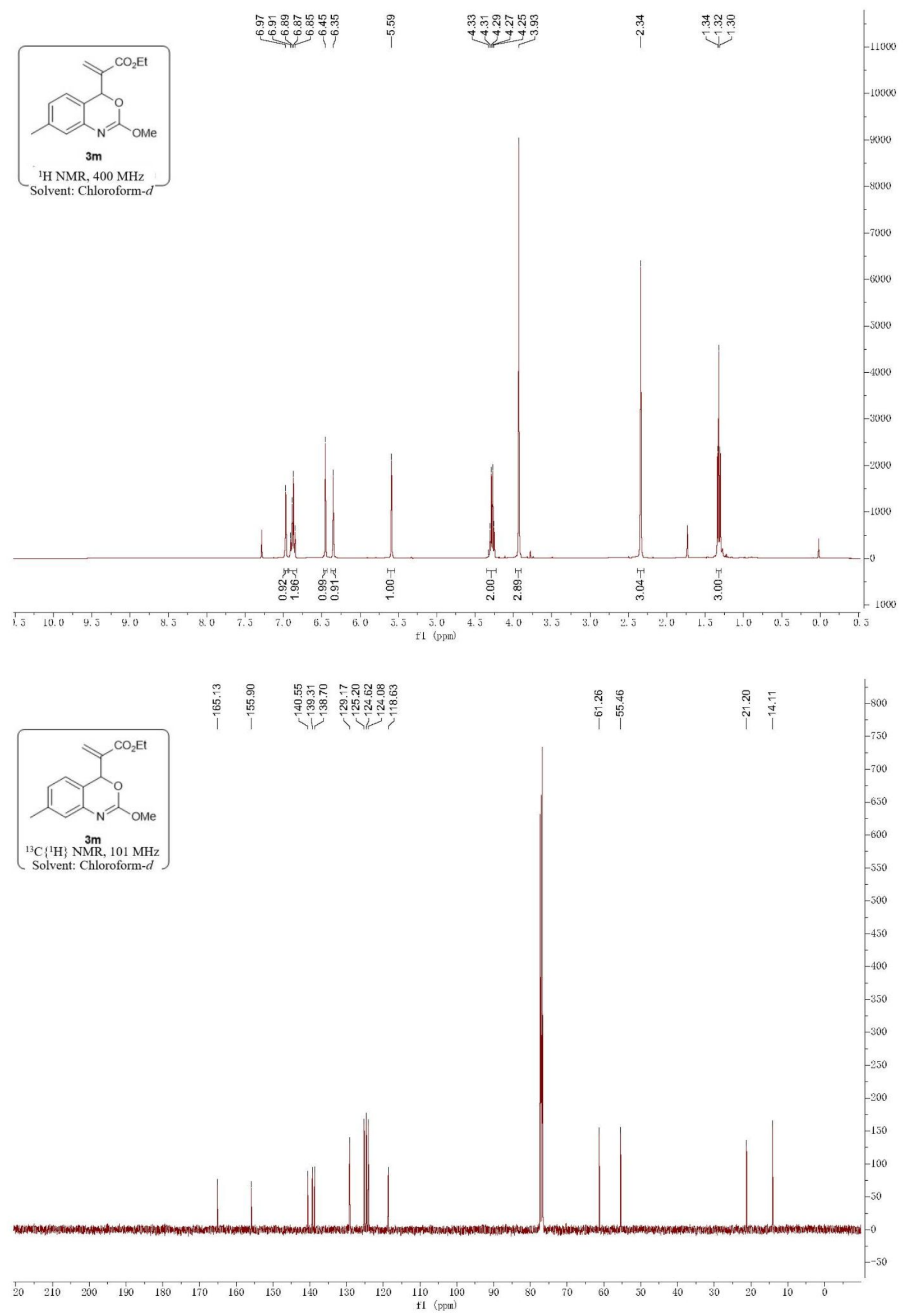

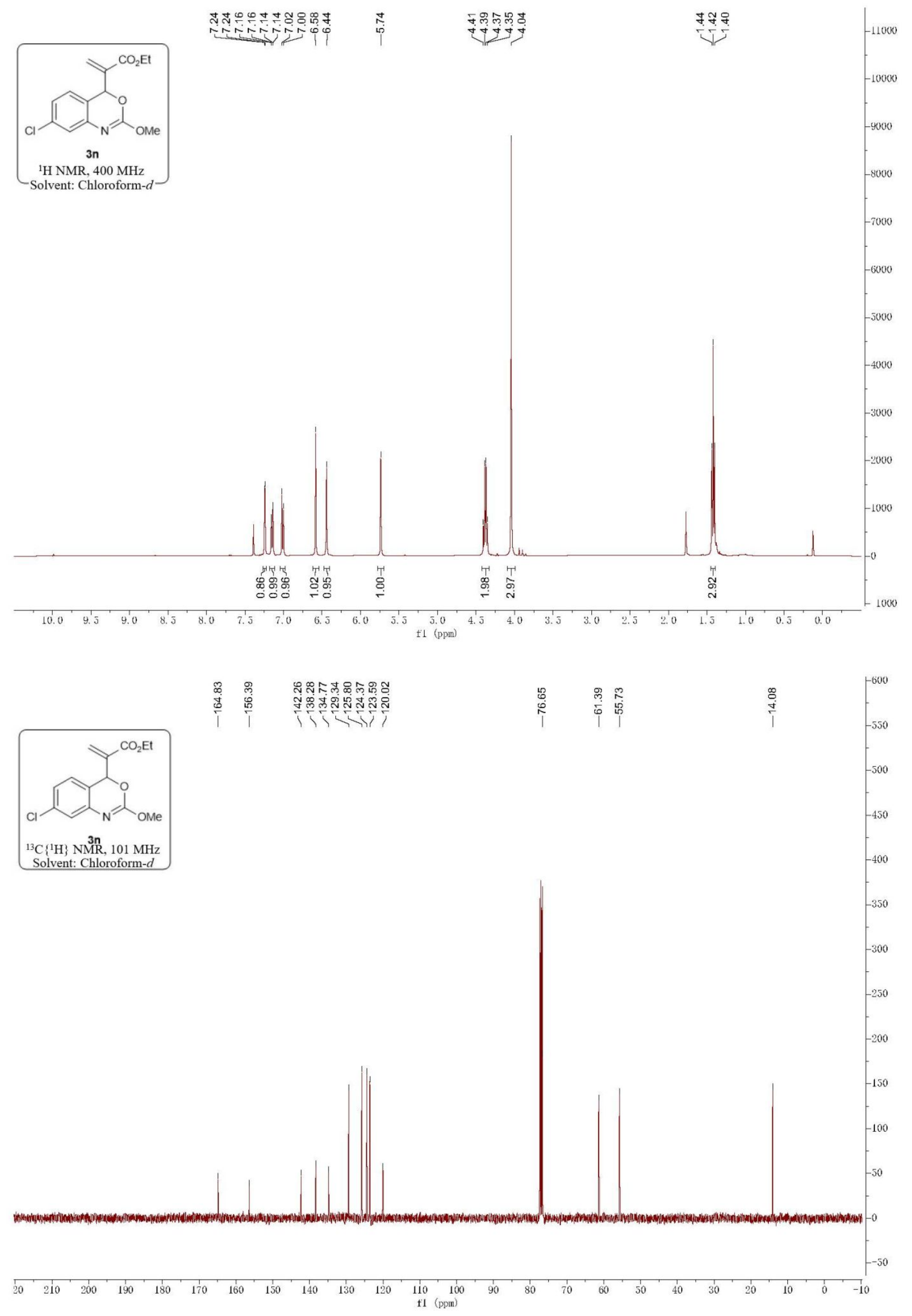

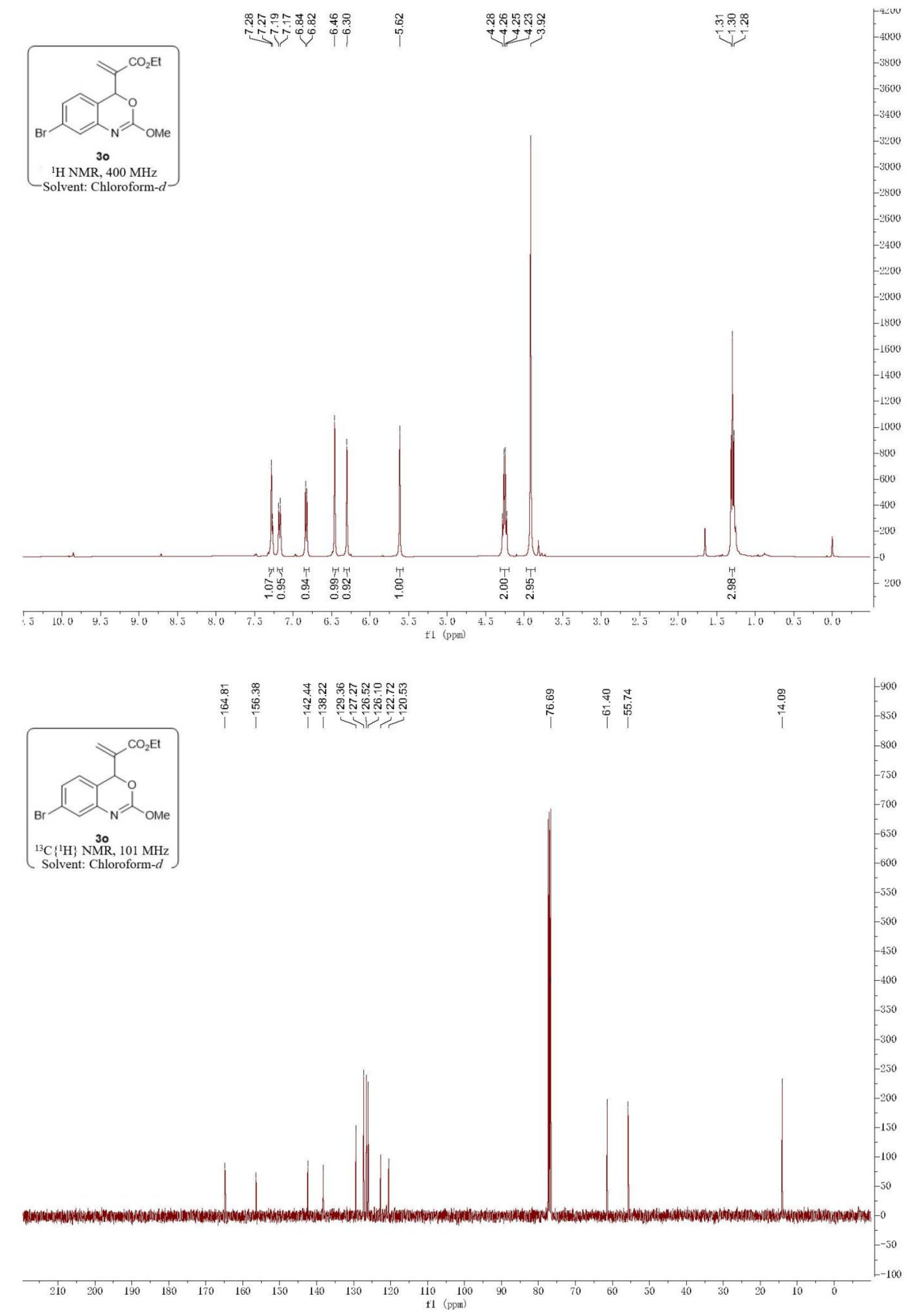


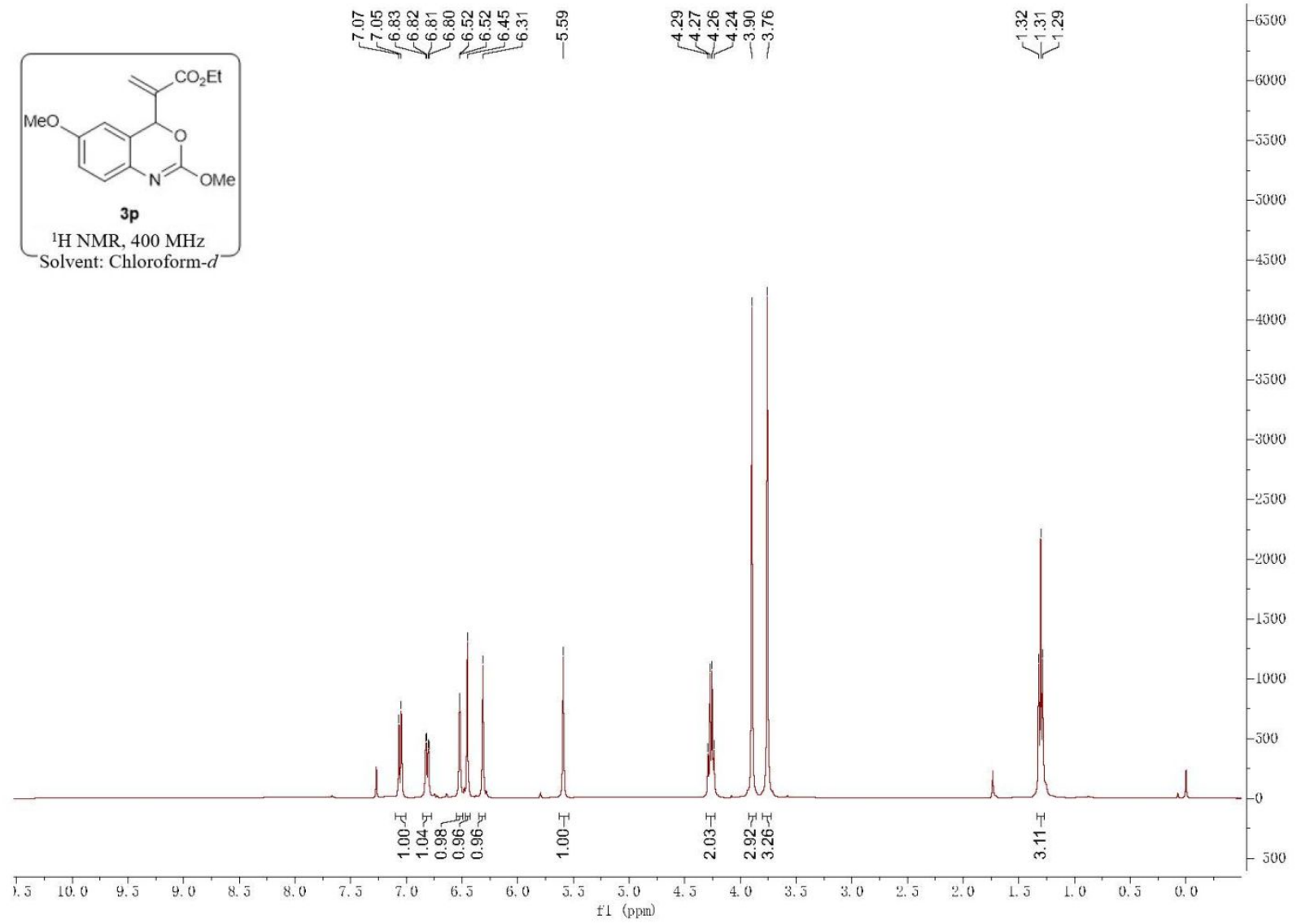

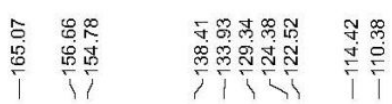

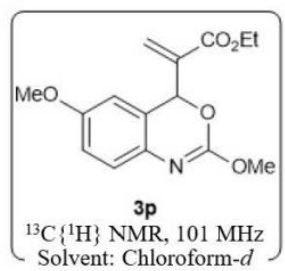

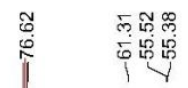



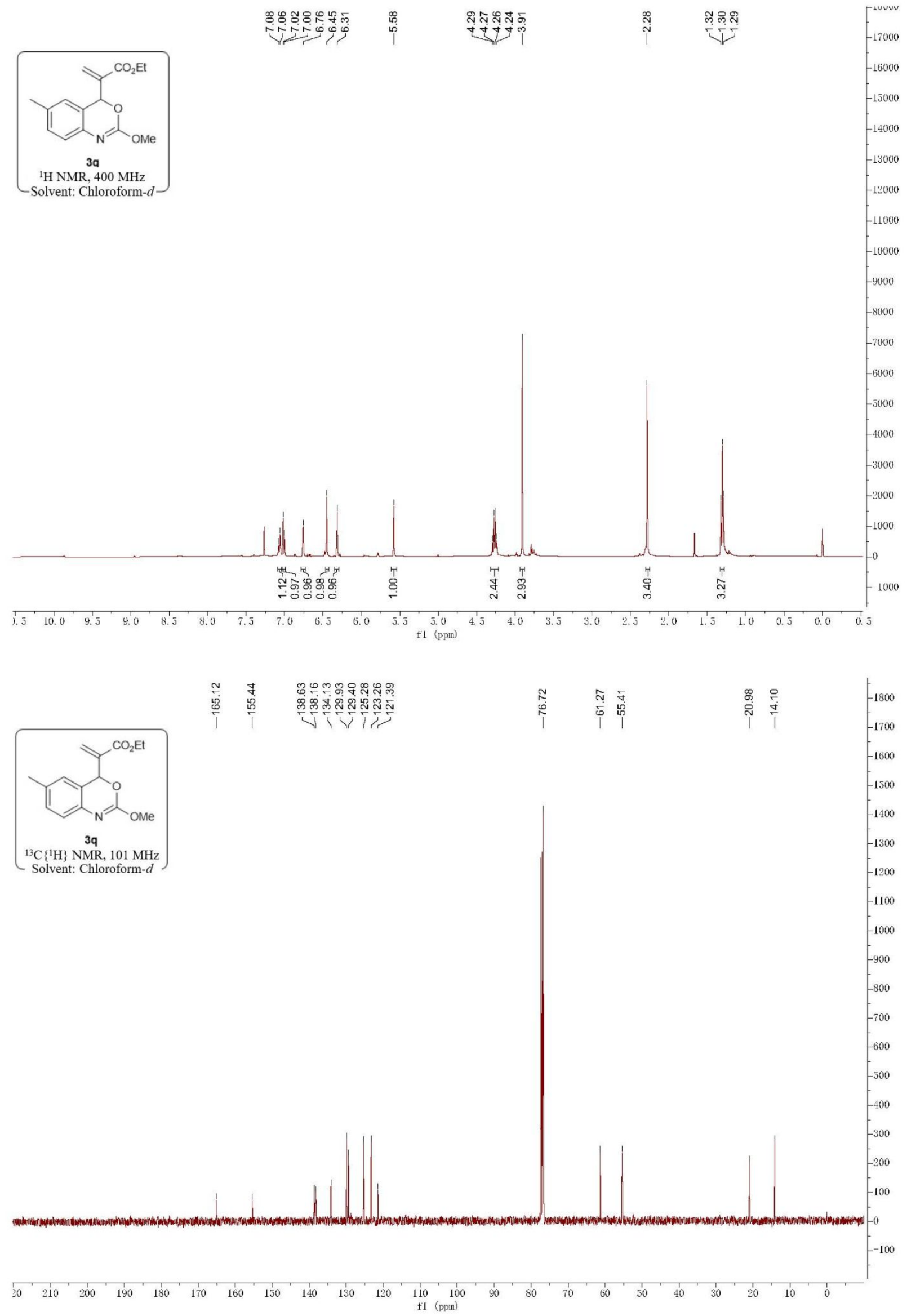

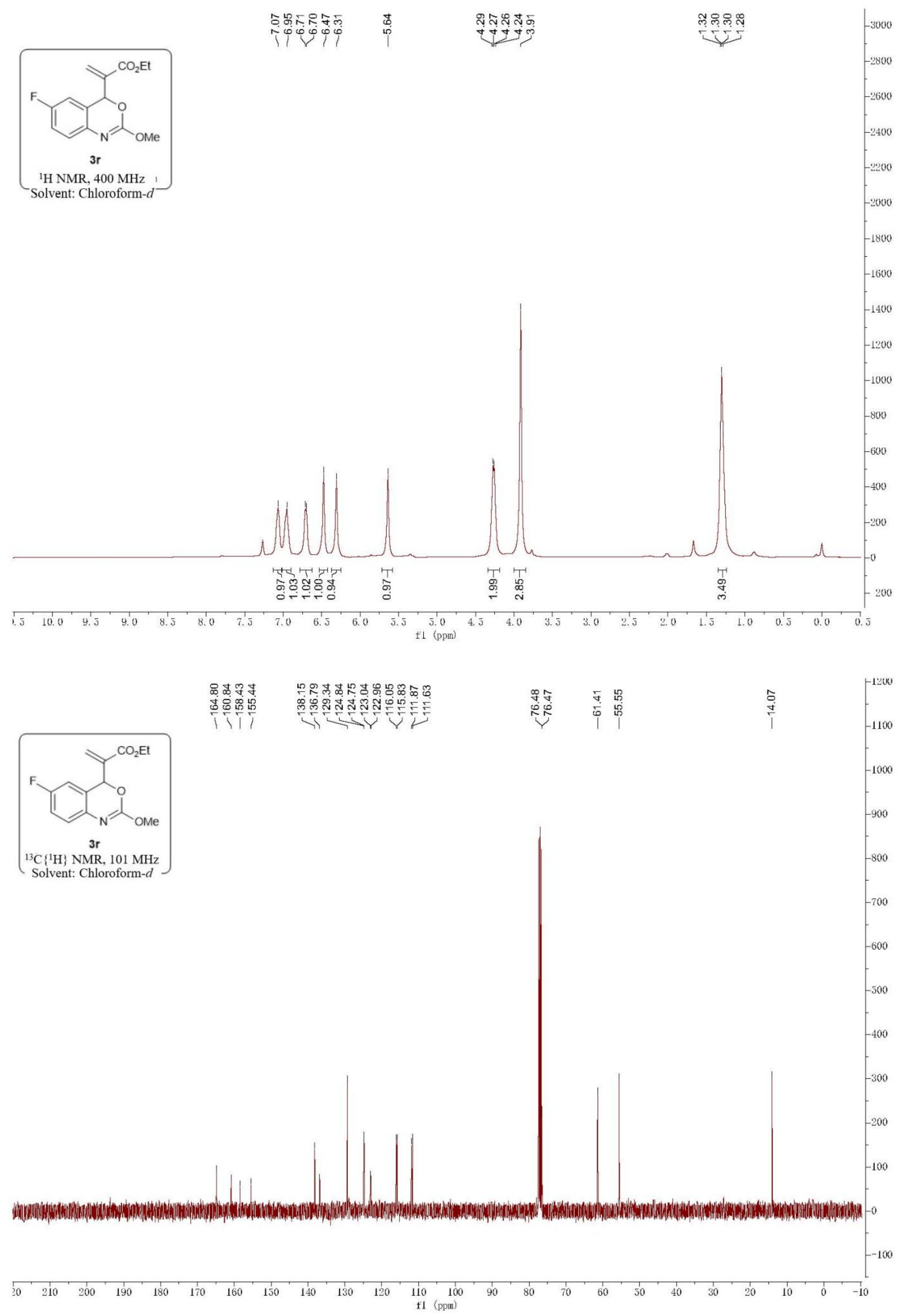

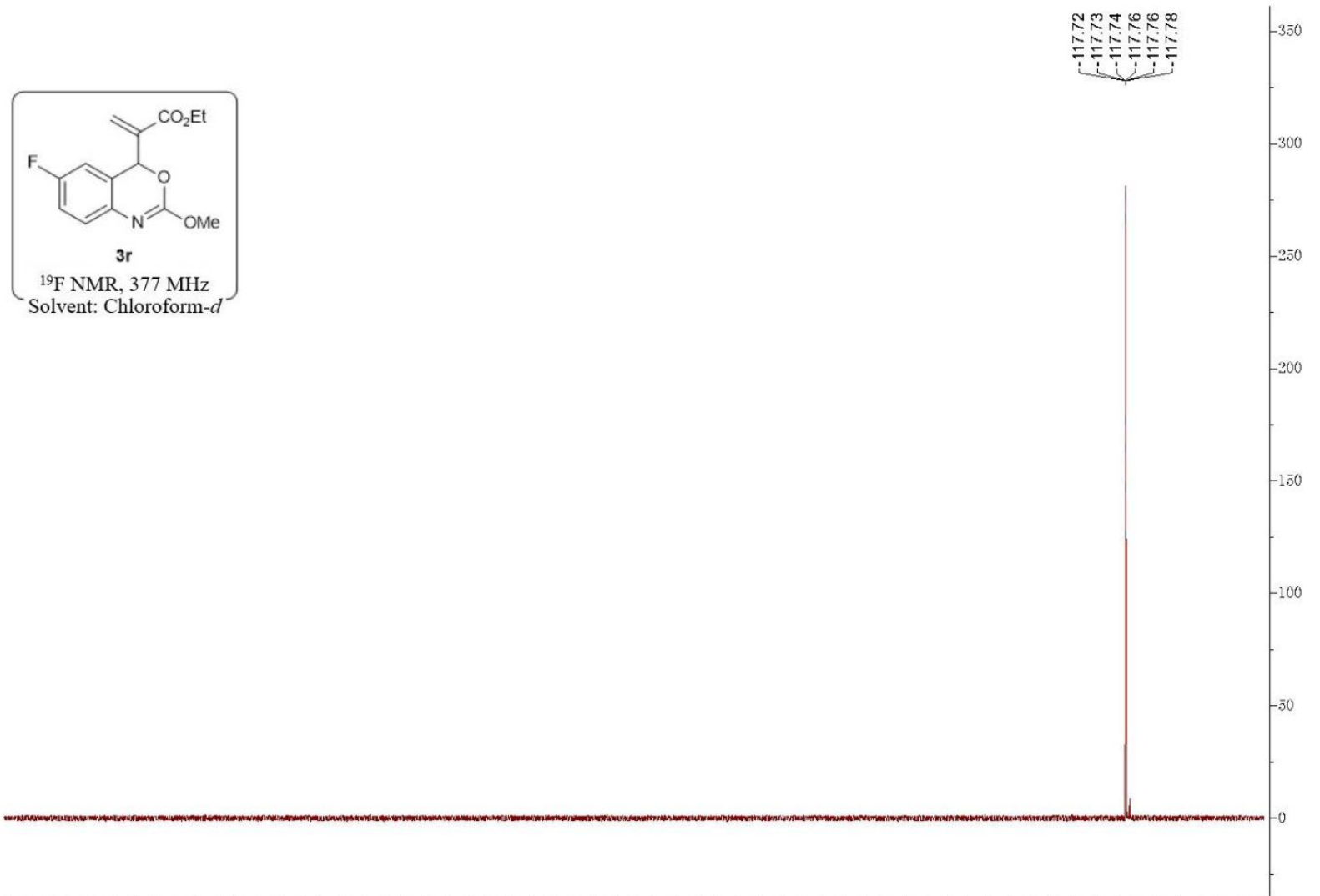

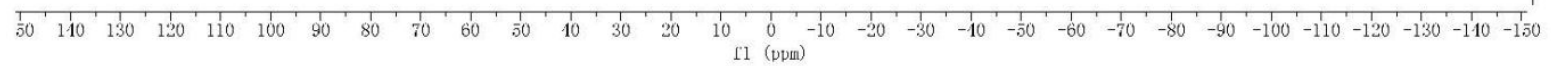

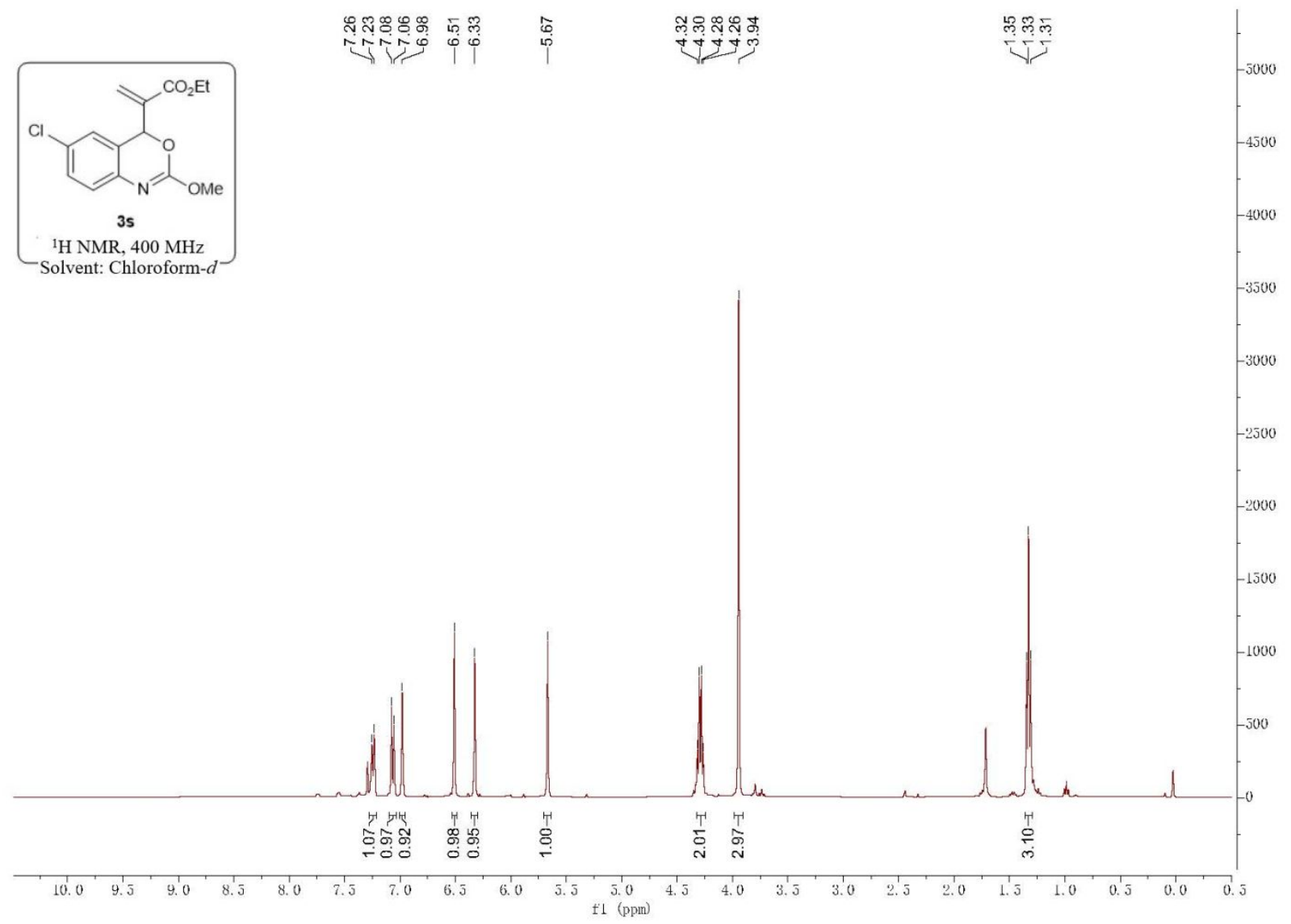




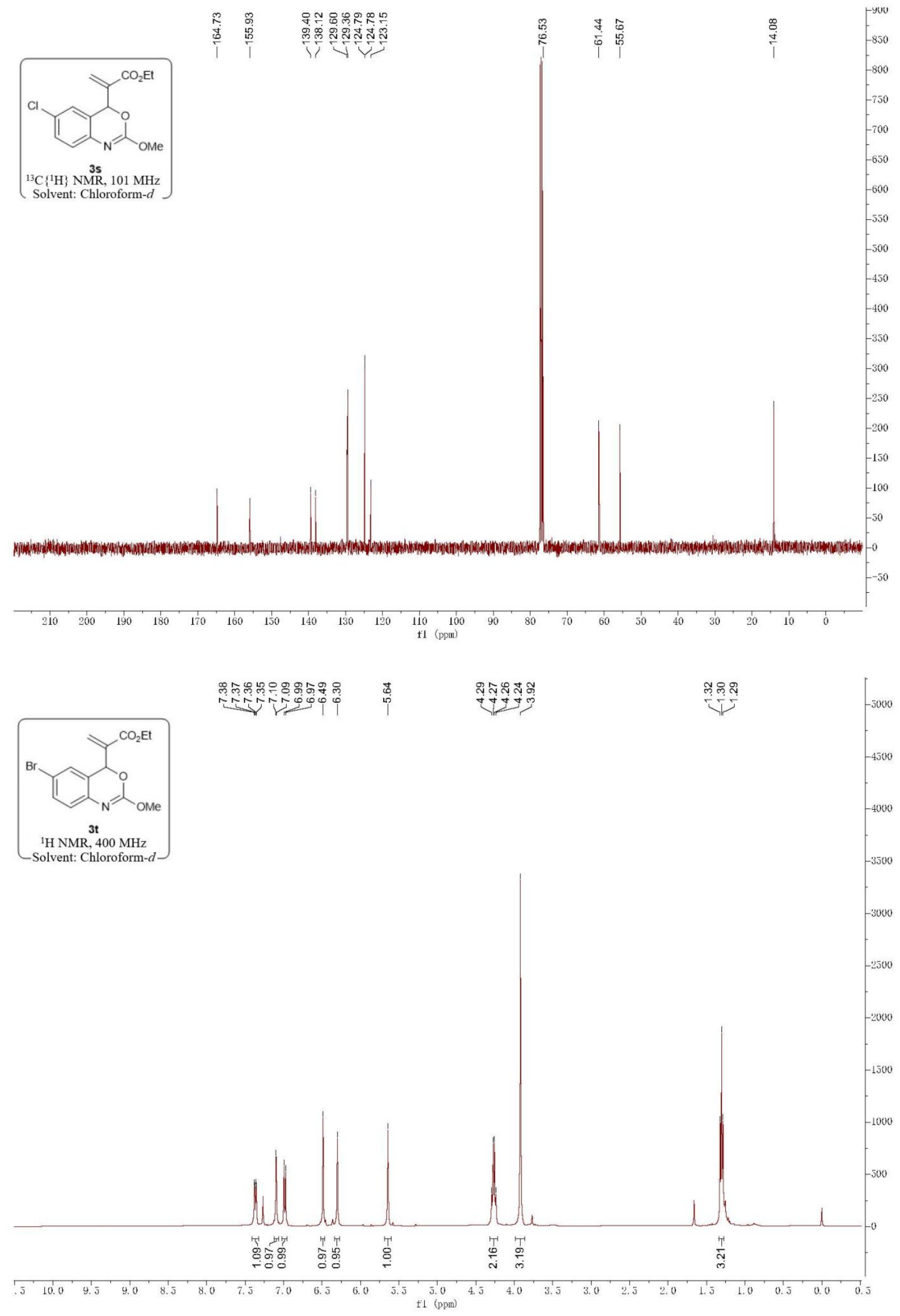




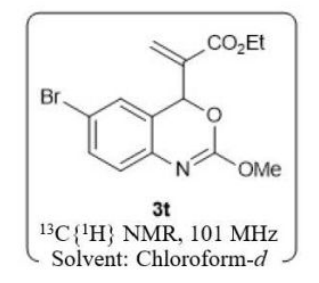

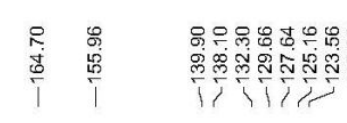

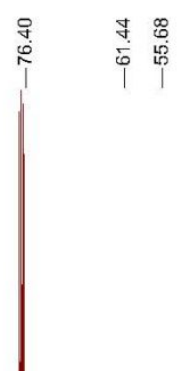

$\underset{\substack{\infty \\ \dot{+}}}{i}$

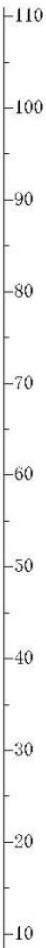

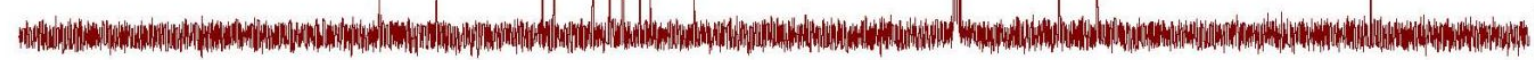

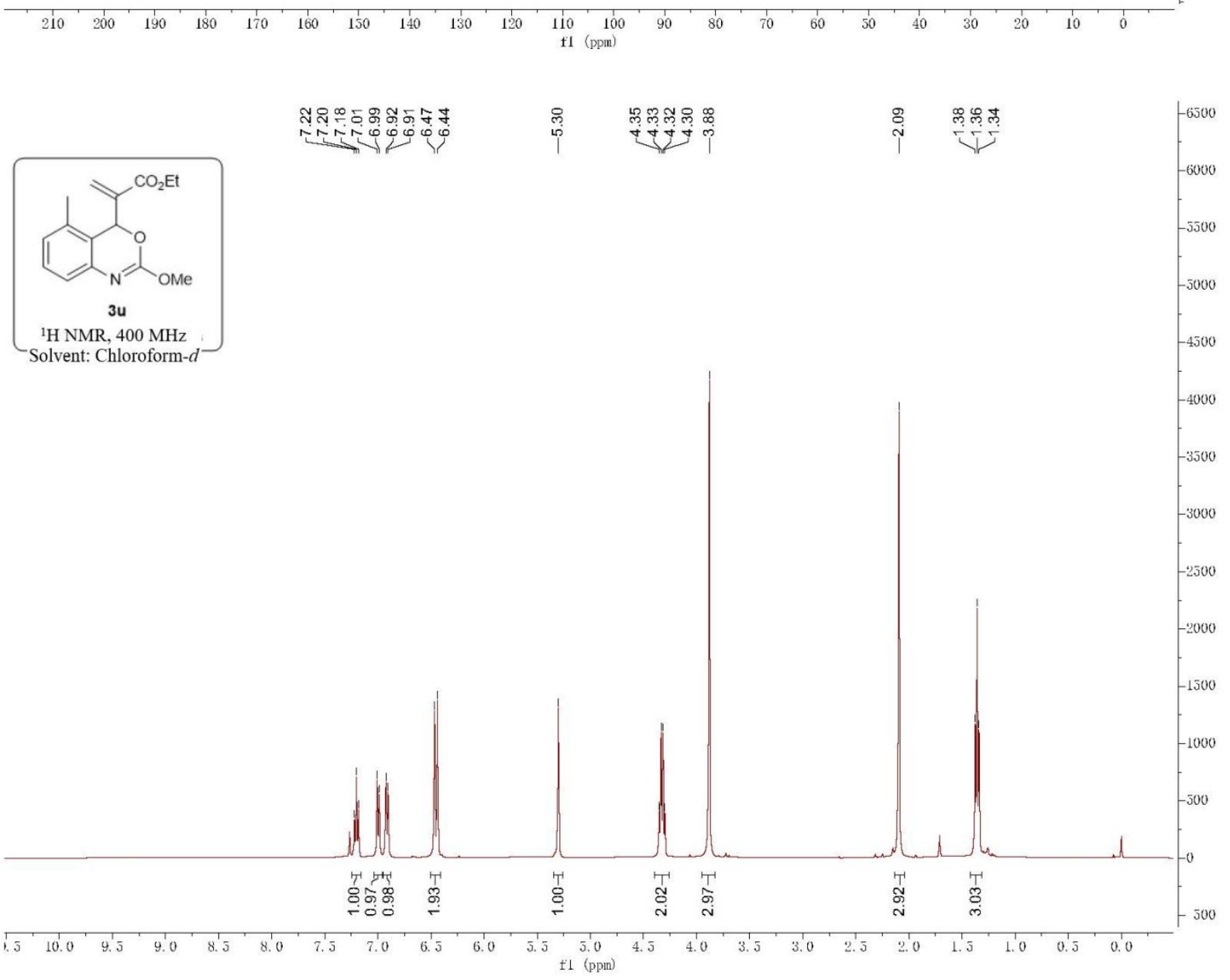




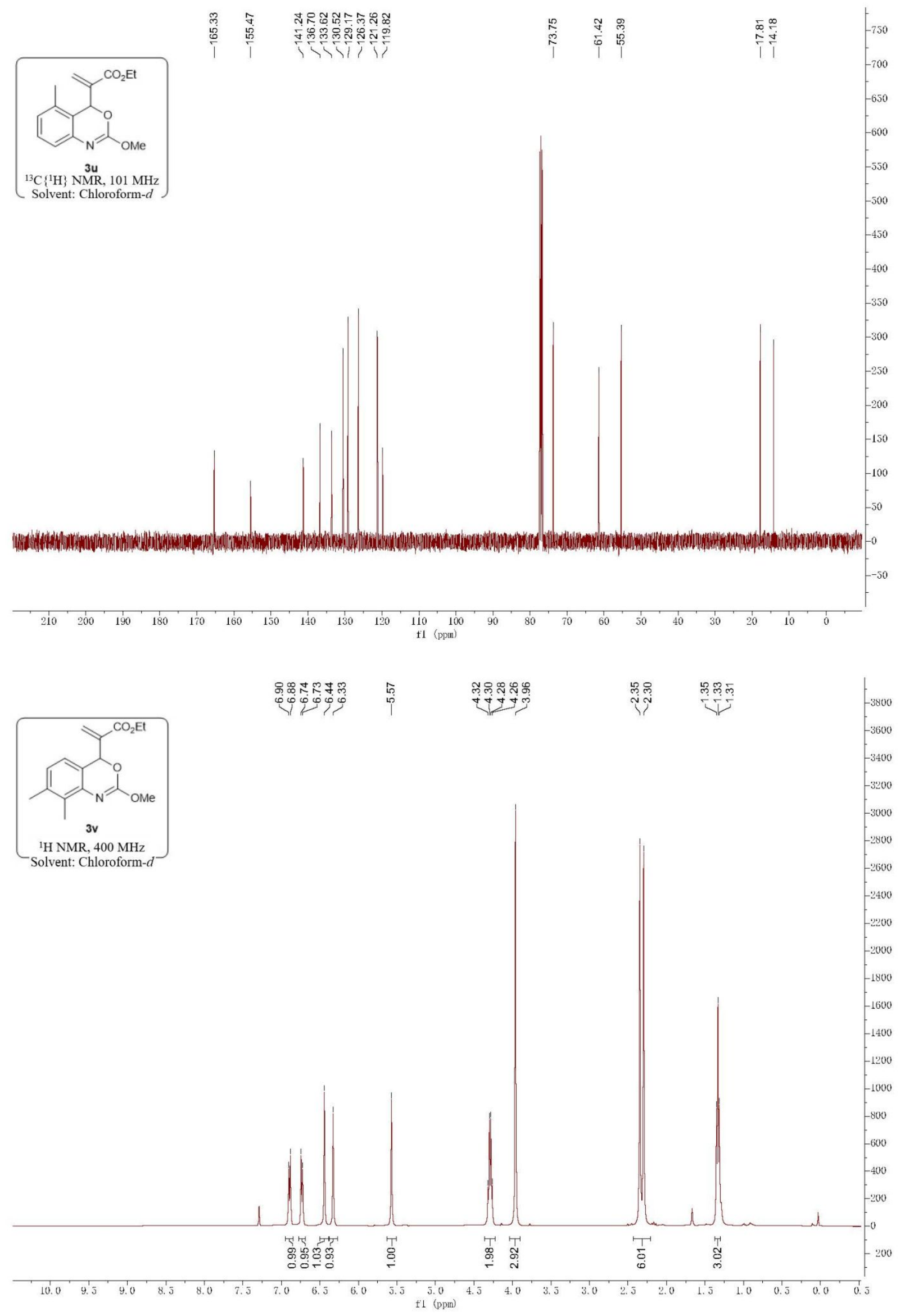




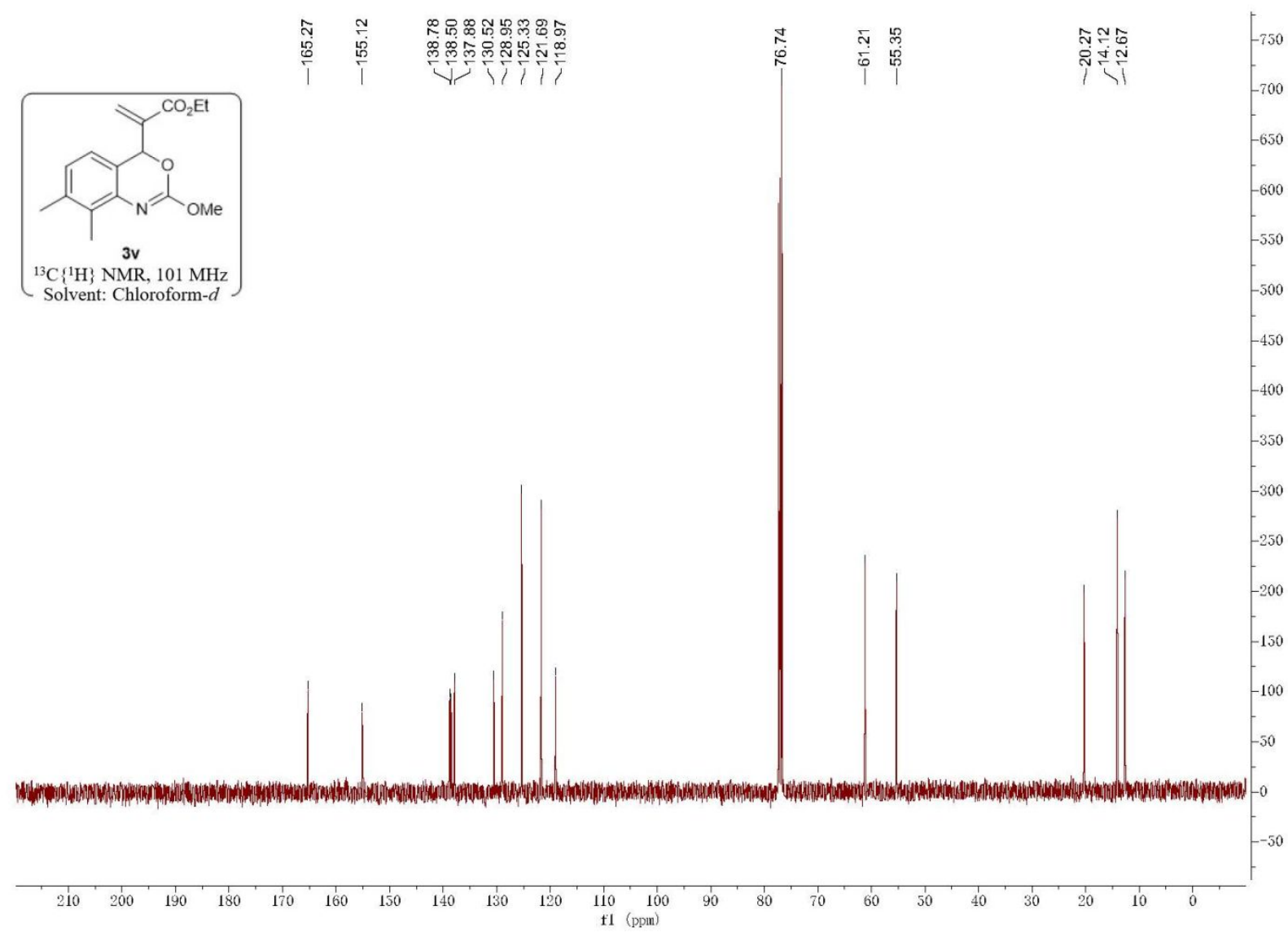


4.4. Products of Derivatization

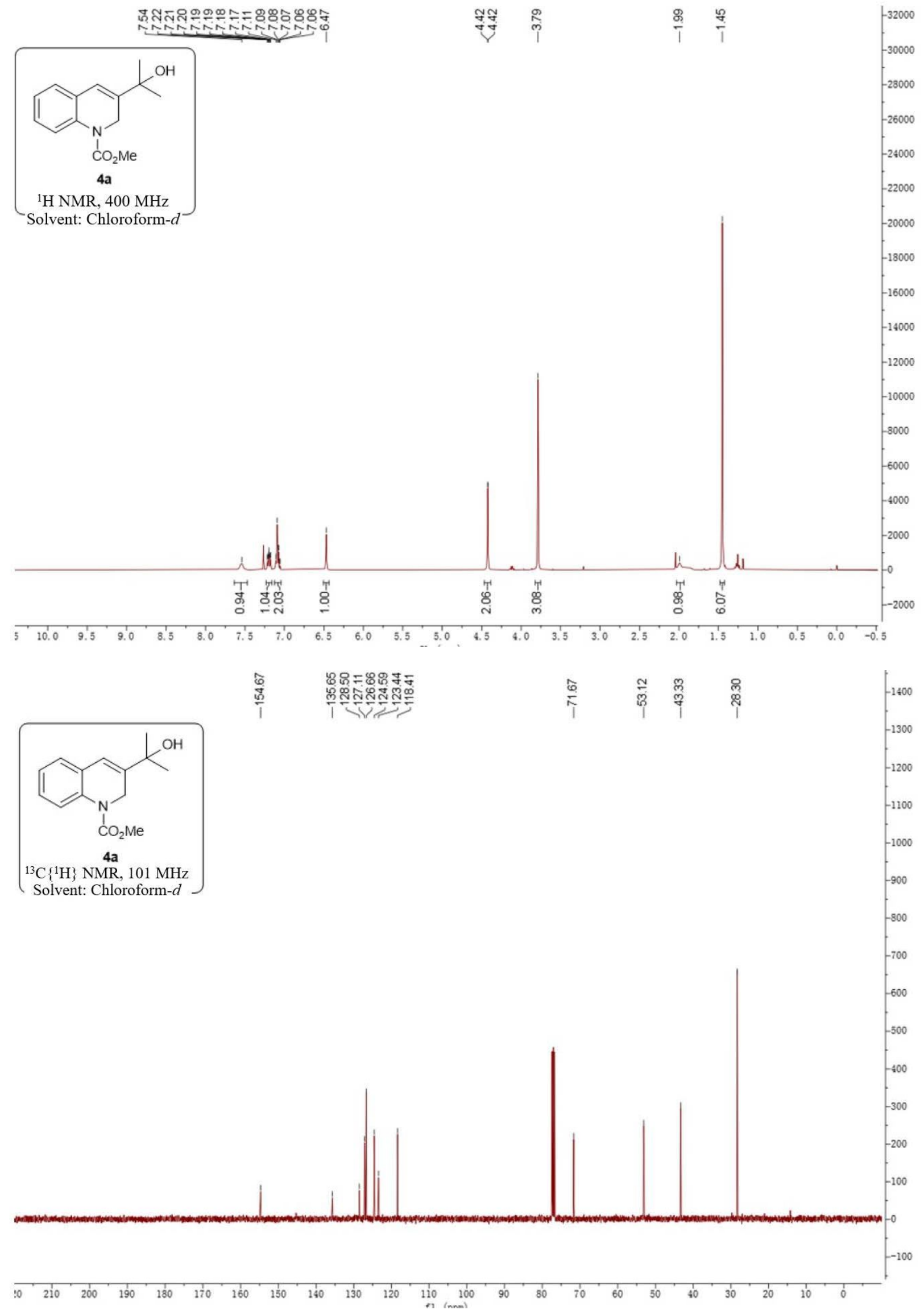



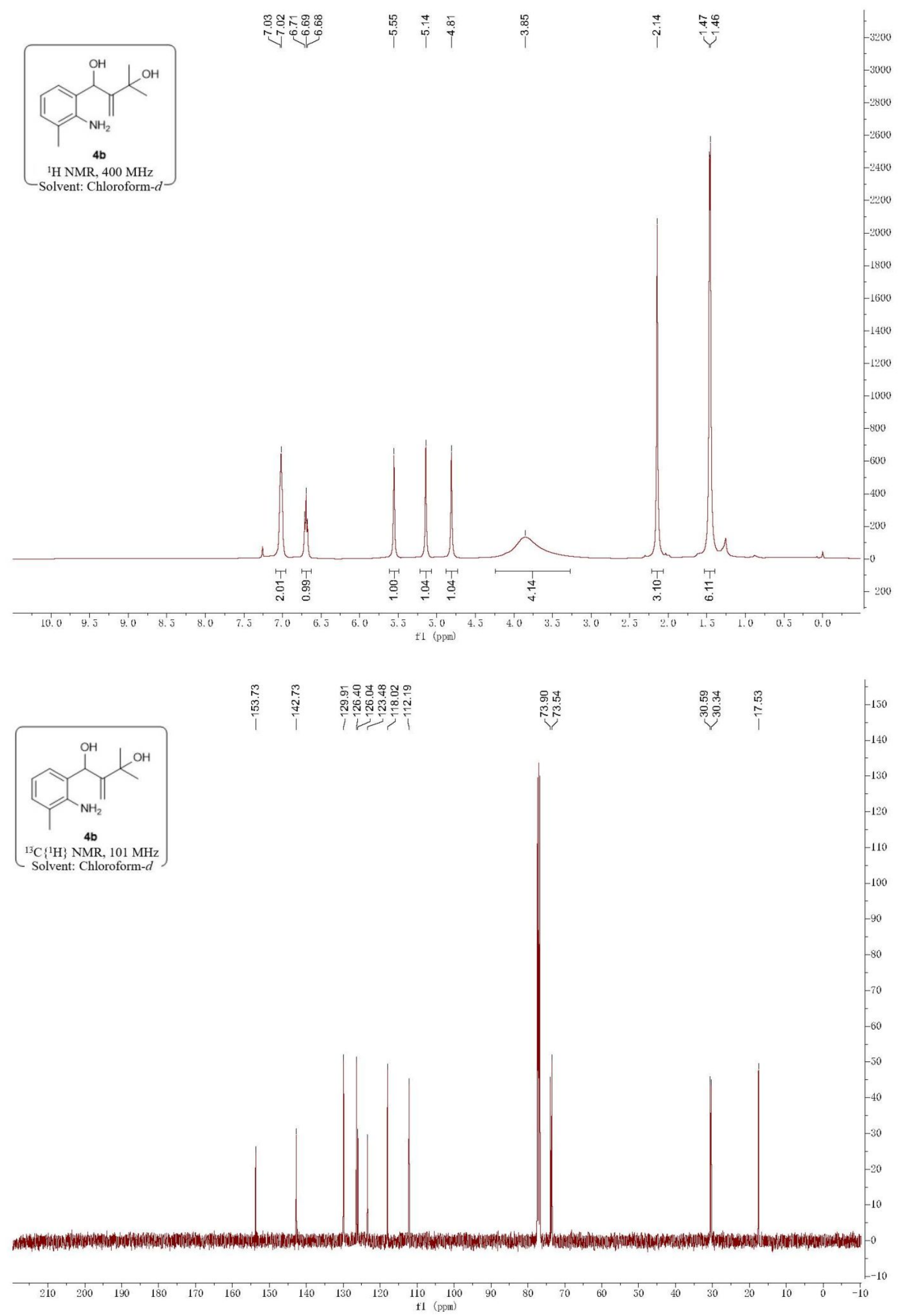

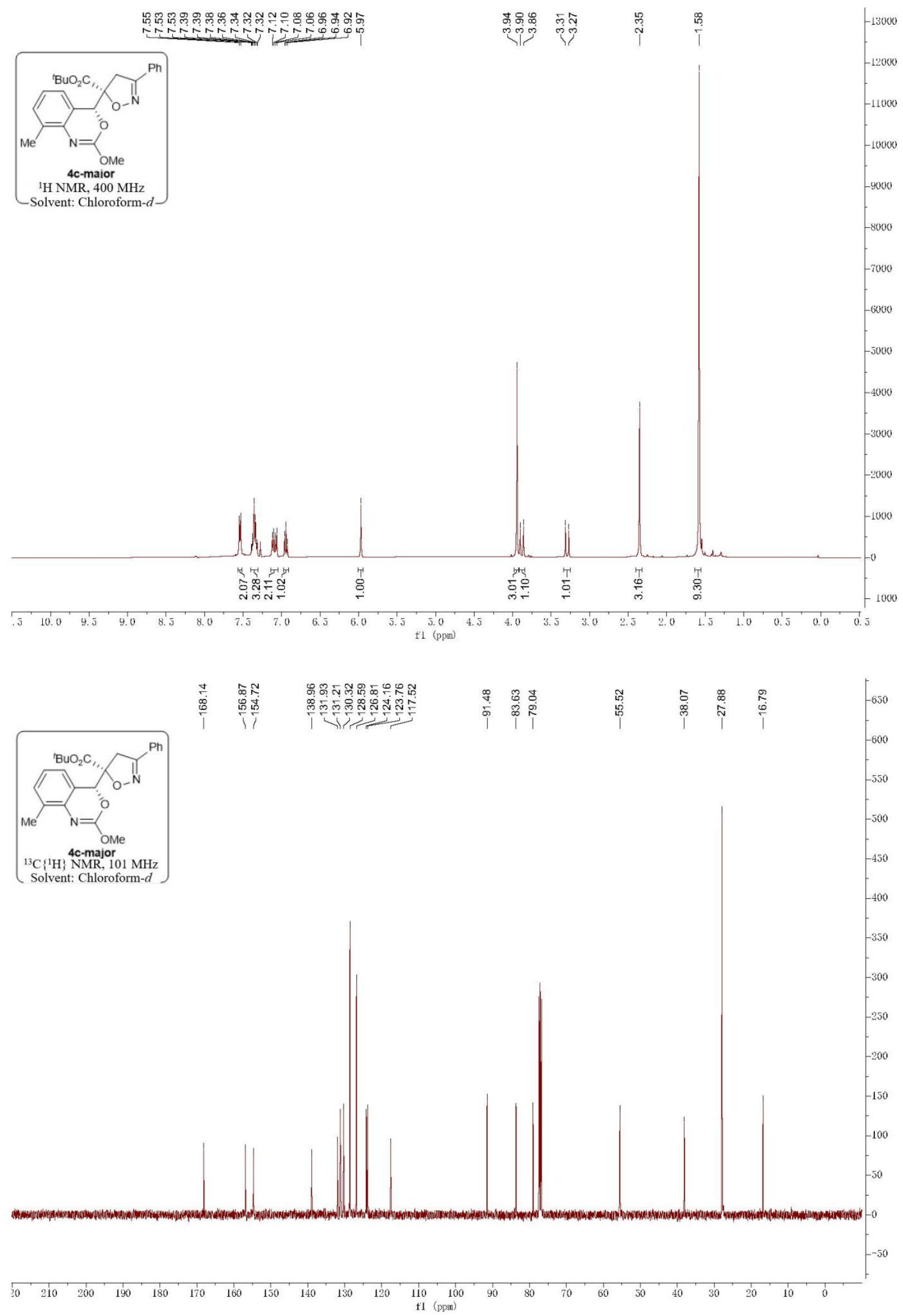

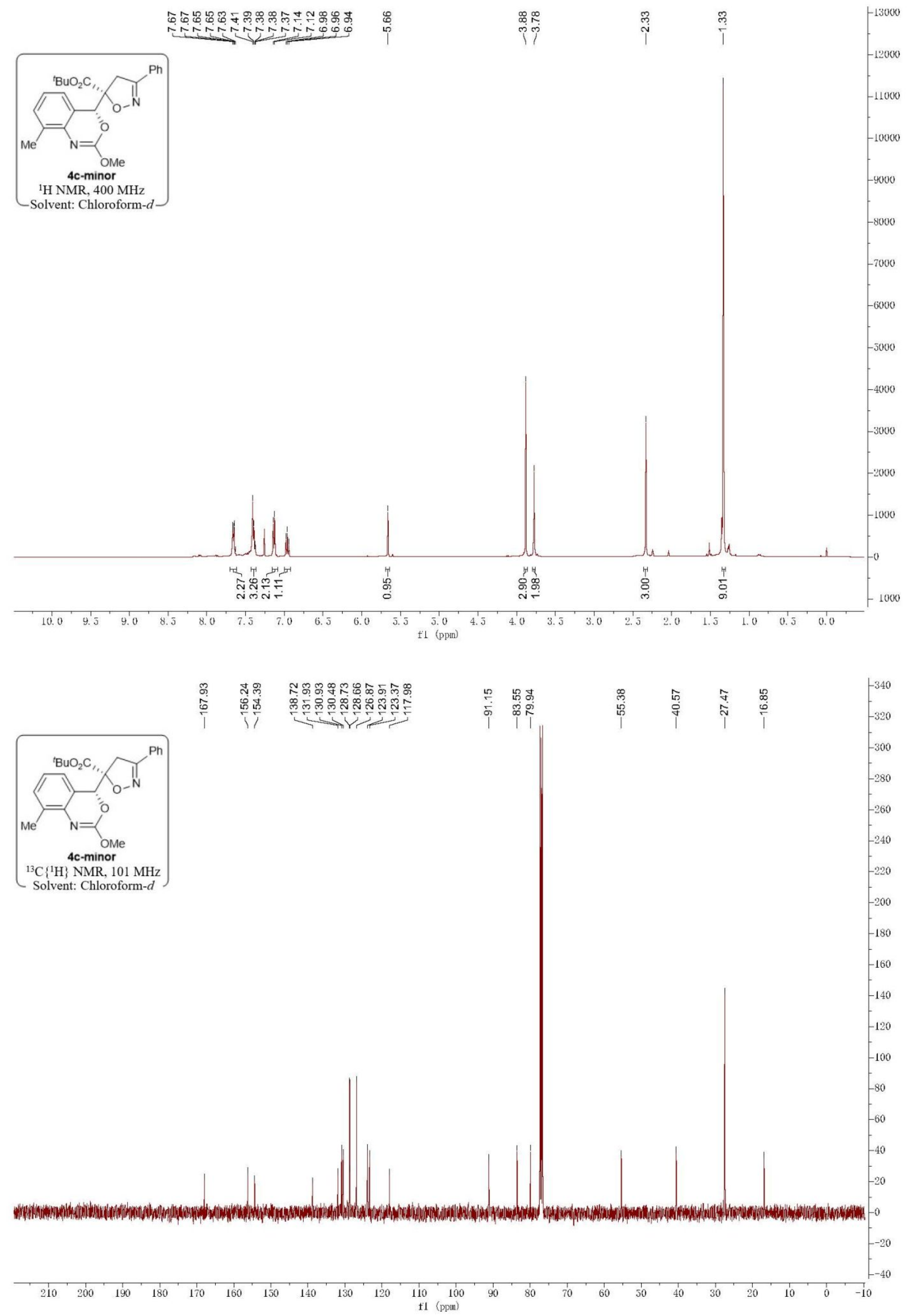

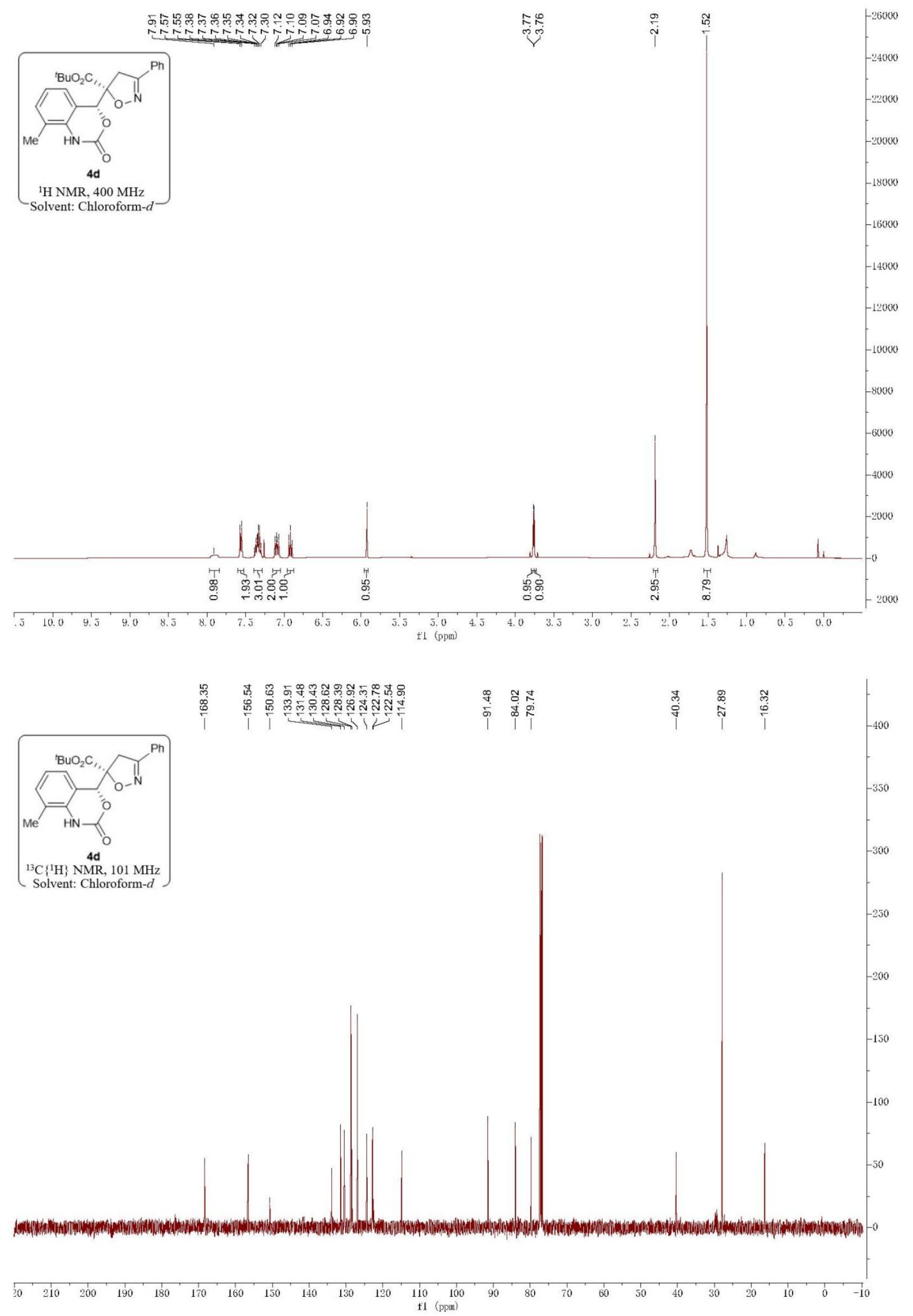

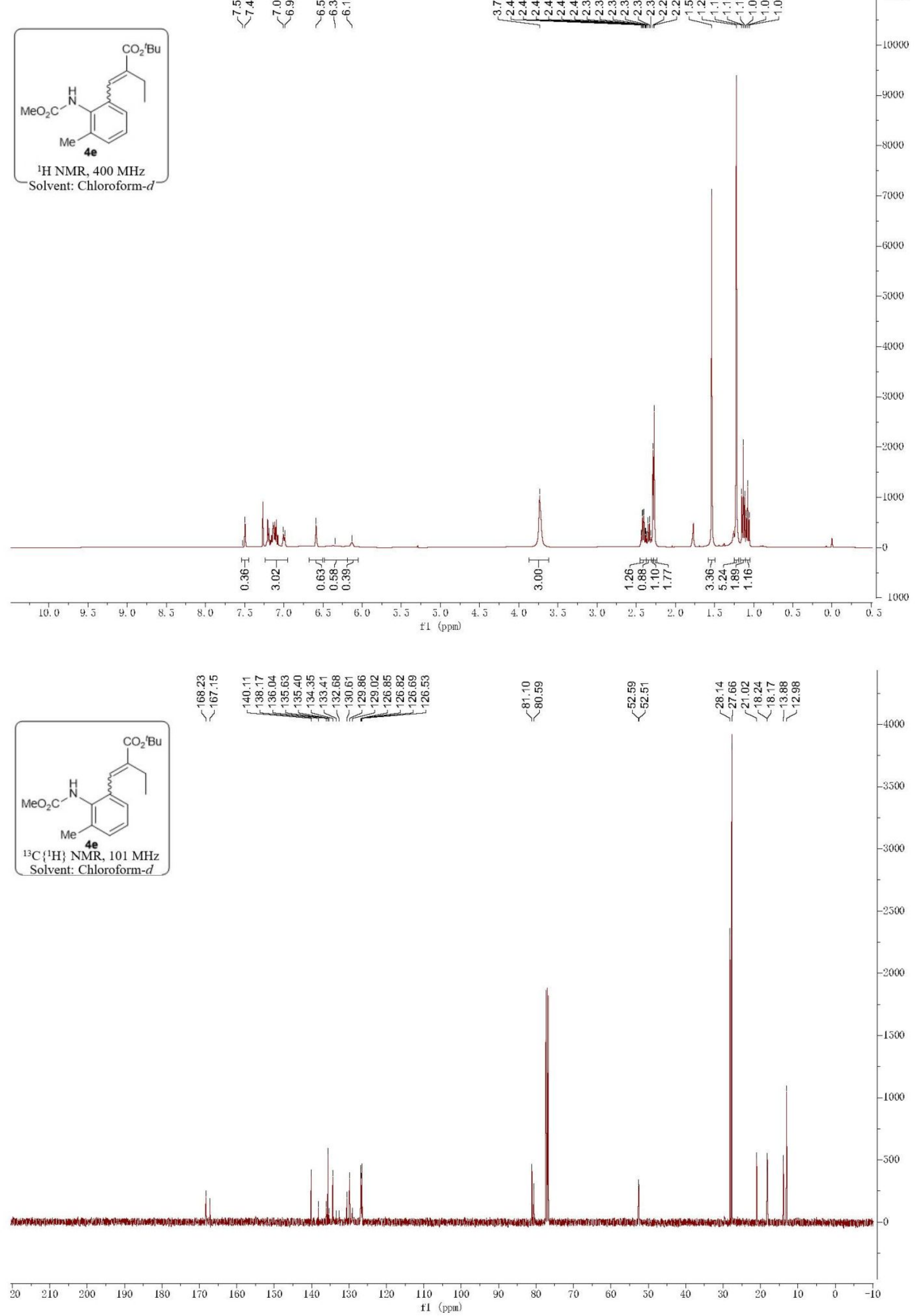

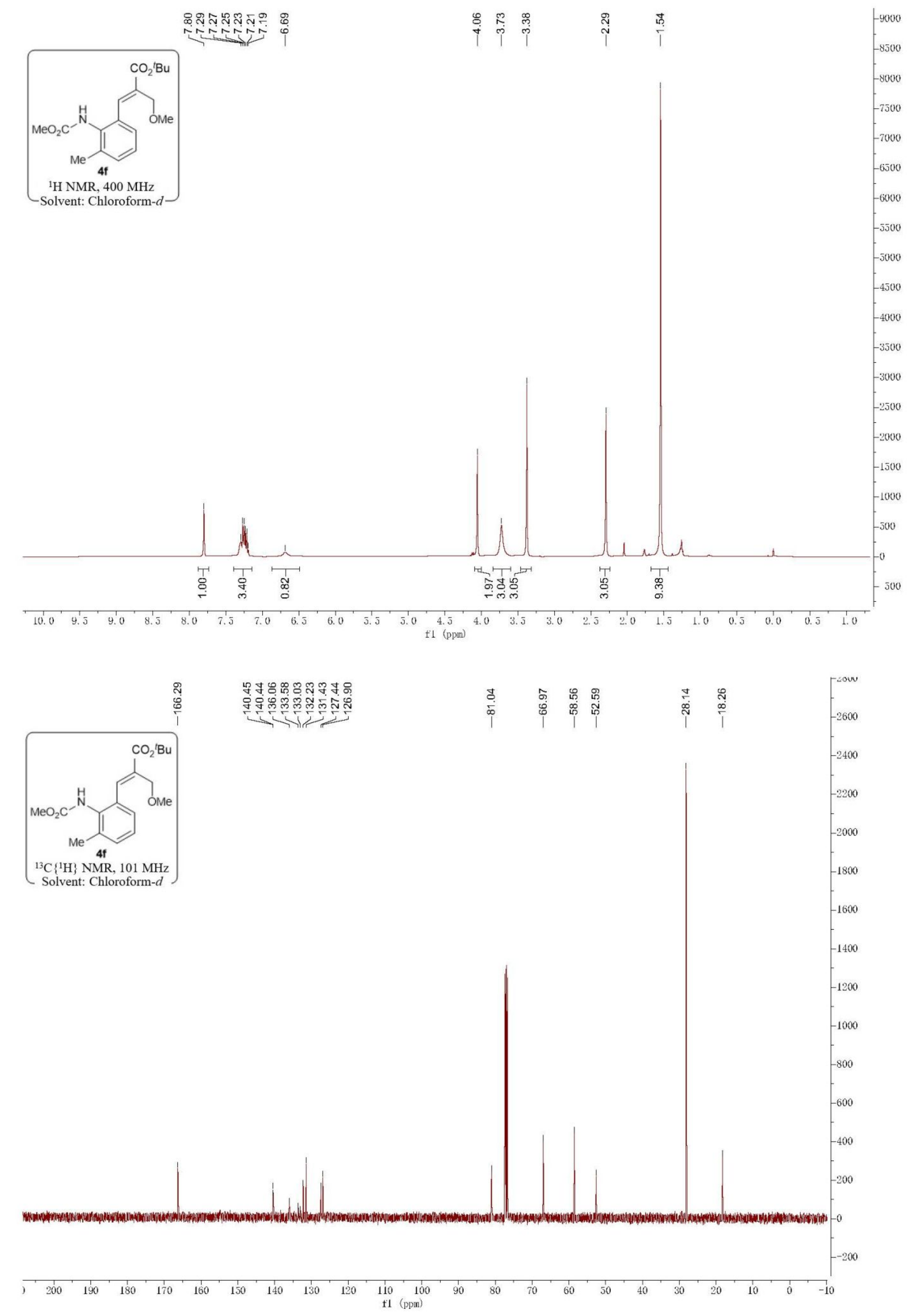

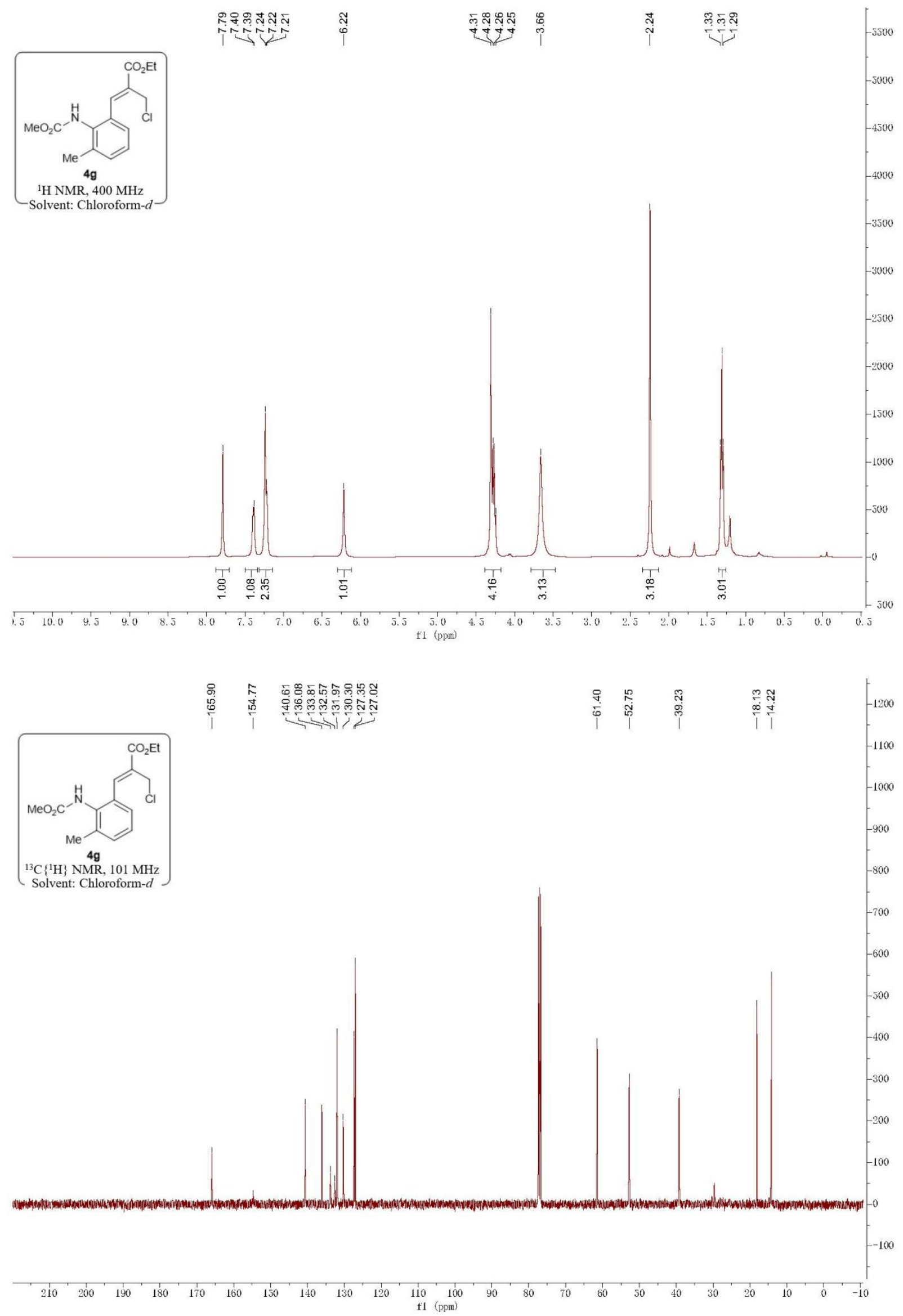